AEI-2009-106

\title{
ELLIPTIC HYPERGEOMETRY OF SUPERSYMMETRIC DUALITIES
}

\author{
V. P. SPIRIDONOV AND G. S. VARTANOV
}

\begin{abstract}
We give a full list of known $\mathcal{N}=1$ supersymmetric quantum field theories related by the Seiberg electric-magnetic duality conjectures for $S U(N), S P(2 N)$ and $G_{2}$ gauge groups. Many of the presented dualities are new, not considered earlier in the literature. For all these theories we construct superconformal indices and express them in terms of elliptic hypergeometric integrals. This gives a systematic extension of the related Römelsberger and Dolan-Osborn results. Equality of indices in dual theories leads to various identities for elliptic hypergeometric integrals. About half of them were proven earlier, and another half represents new challenging conjectures. In particular, we conjecture a dozen new elliptic beta integrals on root systems extending the univariate elliptic beta integral discovered by the first author.
\end{abstract}

\section{Contents}

1. Introduction 2

2. General structure of the elliptic hypergeometric integrals 3

$\begin{array}{lr}3 . & \text { Superconformal index } \\ & 10\end{array}$

$\begin{array}{lr}\text { 3.1. } \mathcal{N}=1 \text { superconformal algebra } & 10\end{array}$

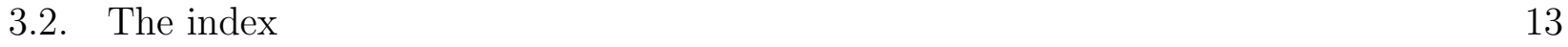

$\begin{array}{ll}\text { 3.3. Calculation of the index } & 15\end{array}$

$\begin{array}{ll}\text { 4. Seiberg duality for unitary gauge groups } & 16\end{array}$

$\begin{array}{ll}\text { 5. Intriligator-Pouliot duality for symplectic gauge groups } & 19\end{array}$

6. Multiple duality for $S P(2 N)$ gauge group $\quad 19$

7. A new $S P(2 N) \leftrightarrow S P(2 M)$ groups duality 21

8. Multiple duality for $S U(2 N)$ gauge group 23

9. Kutasov-Schwimmer type dualities for the unitary gauge group 25

9.1. $S U(N)$ gauge group with the adjoint matter field 26

$\begin{array}{ll}\text { 9.2. Two adjoint matter fields case } & 27\end{array}$

9.3. Generalized KS type dualities 27

9.4. Adjoint, symmetric and conjugate symmetric tensor matter fields 31

9.5. Adjoint, anti-symmetric and conjugate anti-symmetric tensor matter fields $\quad 32$

9.6. Adjoint, anti-symmetric and conjugate symmetric tensor matter fields 34

10. KS type dualities for symplectic gauge groups 35

10.1. The anti-symmetric tensor matter field 35

10.2. Symmetric tensor matter field 36

10.3. Two anti-symmetric tensor matter fields 37

10.4. Symmetric and anti-symmetric tensor matter fields 38

11. Some other new dualities $\quad 39$

11.1. $S U \leftrightarrow S P$ groups mixing duality $\quad 39$

11.2. $S U \leftrightarrow S U$ groups mixing duality $\quad 40$

12. $S$-confinement 40

12.1. $S U(N)$ gauge group $\quad 41$

12.2. Exceptional cases for unitary gauge groups 49 
12.3. Symplectic gauge group $\quad 52$

13. Exceptional $G_{2}$ group $\quad 55$

14. 't Hooft anomaly matching conditions 56

15. Conclusion $\quad 59$

Appendix A. Characters of representations of classical groups 61

Appendix B. Invariant matrix group measures $\quad 63$

Appendix C. Relevant Casimir operators $\quad 63$

Appendix D. Total ellipticity for the KS duality indices $\quad 64$

$\begin{array}{ll}\text { References } & 66\end{array}$

\section{INTRODUCTION}

The main goal of this work consists in merging two fields of recent active research in mathematical physics - the Seiberg duality in supersymmetric field theories $[75,76]$ and the theory of elliptic hypergeometric functions [85]. Seiberg duality is an electric-magnetic duality of certain four dimensional quantum field theories with the symmetry group $G_{s t} \times G \times F$, where the superconformal group $G_{s t}=S U(2,2 \mid 1)$ describes properties of the space-time, $G$ is a local gauge invariance group, and $F$ is a global symmetry flavor group. Conjecturally, such theories are equivalent to each other at their infrared fixed points, existence of which follows from a deeply nontrivial nonperturbative dynamics [47, 79].

The simplest topological characteristics of supersymmetric theories is the Witten index [99]. Its highly nontrivial superconformal generalization was proposed recently by Römelsberger [72, 73] (for $\mathcal{N}=1$ theories) and Kinney et al [49] (for extended supersymmetric theories). These superconformal indices describe the structure of BPS states protected by one supercharge and its conjugate. They can be considered as a kind of partition functions in the corresponding Hilbert space. Starting from early work [80,95], it is known that such partition functions are described by matrix integrals over the classical groups. The central conjecture of Römelsberger [73] claims the equality of superconformal indices in the Seiberg dual theories. In an interesting work [26], Dolan and Osborn have found an explicit form of these indices for a number of theories and discovered that they coincide with particular examples of the elliptic hypergeometric integrals [89]. This identification allowed them to prove Römelsberger's conjecture for several dualities either on the basis of known exact computability of these integrals or from the existence of non-trivial symmetry transformations for them.

The general notion of elliptic hypergeometric integrals was introduced by the first author in [81, 83]. First example of such integrals discovered in [81] has formed a new class of exactly computable integrals of hypergeometric type called elliptic beta integrals. Such a name was chosen because these integrals can be considered as a top level generalization of the well-known Euler beta integral [1]:

$$
\int_{0}^{1} x^{\alpha-1}(1-x)^{\beta-1} d x=\frac{\Gamma(\alpha) \Gamma(\beta)}{\Gamma(\alpha+\beta)}, \quad \operatorname{Re} \alpha, \operatorname{Re} \beta>0,
$$

where $\Gamma(x)$ is the Euler gamma function. Elliptic hypergeometric functions generalize known plain hypergeometric functions and their $q$-analogues [1]. Moreover, their properties have clarified the origins of many old notions of the hypergeometric world [82]. Limits of the elliptic hypergeometric integrals (or of the elliptic hypergeometric series hidden behind them) matched with the elliptic curve degenerations brought to light new types of $q$-hypergeometric functions as well $[66,67]$ (see also [10]). 
In the present work (which was initiated in August 2008 after the first author has known [26]), we extend systematically the Römelsberger and Dolan-Osborn results. More precisely, we present a full list of known $\mathcal{N}=1$ superconformal field theories related by the duality conjecture for simple gauge groups $G=S U(N), S P(2 N), G_{2}$. For all of them we express superconformal indices in terms of the elliptic hypergeometric integrals. Using Seiberg dualities established earlier in the literature (see references below) we come to a large number of identities for elliptic hypergeometric integrals. About half of them were proven earlier, which yields a justification of the corresponding dualities. A part of the emerging relations for indices was described in [26], and we prove equalities of superconformal indices for many other dualities. Another half of the constructed identities represents new challenging conjectures requiring rigorous mathematical proof. We give indications how some of them can be proved with the help of hypergeometric techniques.

Remarkably, from known relations for elliptic hypergeometric integrals we find many new dualities not considered earlier in the literature. Thus we describe both new elliptic hypergeometric identities and new $\mathcal{N}=1$ supersymmetric theories obeying an electric-magnetic duality. In particular, we conjecture more than ten new elliptic beta integrals on root systems, which extend the univariate elliptic beta integral of [81].

Analyzing the general structure of all relations for integrals in this paper, we formulate two universal conjectures. Namely, we argue that for the existence of a non-trivial identity for an elliptic hypergeometric integral it is necessary to have a so-called totally elliptic hypergeometric term $[82,86,90]$. The second conjecture claims that the same total ellipticity (and related modular invariance) is responsible for the validity of 't Hooft anomaly matching conditions [40], which are fulfilled for all our dualities (the old and new ones).

A detailed consideration of the multiple duality phenomenon for $G=S P(2 N)$ gauge group case and a brief announcement of other results of this work were given in paper [91]. Our results were reported also at IV-th Sakharov Conference on Physics (Lebedev Institute, Moscow, May 2009), Conformal Field Theory Workshop (Landau Institute, Chernogolovka, June 2009), XVIth International Congress on Mathematical Physics (Prague, August 2009), and about ten seminars at different institutes. We thank the organizers of these meetings and seminars for invitations and kind hospitality.

\section{General structure of the elliptic hypergeometric integrals}

We start our consideration from the description of the general structure of elliptic hypergeometric integrals. For any $x \in \mathbb{C}$ and a base $p \in \mathbb{C},|p|<1$, we define the infinite product

$$
(x ; p)_{\infty}=\prod_{j=0}^{\infty}\left(1-x p^{j}\right) .
$$

Then the theta function is defined as

$$
\theta(x ; p)=(x ; p)_{\infty}\left(p x^{-1} ; p\right)_{\infty}
$$

where $x \in \mathbb{C}^{*}$. This function obeys the symmetry properties

$$
\theta\left(x^{-1} ; p\right)=\theta(p x ; p)=-x^{-1} \theta(x ; p)
$$

and the addition law

$$
\theta\left(x w^{ \pm 1}, y z^{ \pm 1} ; p\right)-\theta\left(x z^{ \pm 1}, y w^{ \pm 1} ; p\right)=y w^{-1} \theta\left(x y^{ \pm 1}, w z^{ \pm 1} ; p\right)
$$

where $x, y, w, z \in \mathbb{C}^{*}$ and we use the convention

$$
\theta\left(x_{1}, \ldots, x_{k} ; p\right):=\theta\left(x_{1} ; p\right) \ldots \theta\left(x_{k} ; p\right), \quad \theta\left(t x^{ \pm 1} ; p\right):=\theta\left(t x, t x^{-1} ; p\right) .
$$


The Jacobi triple product identity for the standard theta series yields

$$
\theta(x ; p)=\frac{1}{(p ; p)_{\infty}} \sum_{n \in \mathbb{Z}} p^{n(n-1) / 2}(-x)^{n} .
$$

For arbitrary $q \in \mathbb{C}$ and $n \in \mathbb{Z}$, we introduce the elliptic shifted factorials

$$
\theta(x ; p ; q)_{n}:= \begin{cases}\prod_{j=0}^{n-1} \theta\left(x q^{j} ; p\right), & \text { for } n>0 \\ \prod_{j=1}^{-n} \theta\left(x q^{-j} ; p\right)^{-1}, & \text { for } n<0\end{cases}
$$

with the normalization $\theta(x ; p ; q)_{0}=1$. For $p=0$ we have $\theta(x ; 0)=1-x$ and

$$
\theta(x ; 0 ; q)_{n}=(x ; q)_{n}=(1-x)(1-q x) \cdots\left(1-q^{n-1} x\right),
$$

the standard $q$-Pochhammer symbol [1].

For arbitrary $m \in \mathbb{Z}$, we have the quasiperiodicity relations

$$
\begin{aligned}
& \theta\left(p^{m} x ; p\right)=(-x)^{-m} p^{-\frac{m(m-1)}{2}} \theta(x ; p), \\
& \theta\left(p^{m} x ; p ; q\right)_{k}=(-x)^{-m k} q^{-\frac{m k(k-1)}{2}} p^{-\frac{k m(m-1)}{2}} \theta(x ; p ; q)_{k}, \\
& \theta\left(x ; p ; p^{m} q\right)_{k}=(-x)^{-\frac{m k(k-1)}{2}} q^{-\frac{m k(k-1)(2 k-1)}{6}} p^{-\frac{m k(k-1)}{4}\left(\frac{m(2 k-1)}{3}-1\right)} \theta(x ; p ; q)_{k} .
\end{aligned}
$$

We relate bases $p, q$ and $r$ with three complex numbers $\omega_{1,2,3} \in \mathbb{C}$ in the following way

$$
q=e^{2 \pi \mathrm{i} \frac{\omega_{1}}{\omega_{2}}}, \quad p=e^{2 \pi \mathrm{i} \frac{\omega_{3}}{\omega_{2}}}, \quad r=e^{2 \pi \mathrm{i} \frac{\omega_{3}}{\omega_{1}}} .
$$

Their " $\tau \rightarrow-1 / \tau$ " modular transformed partners are

$$
\tilde{q}=e^{-2 \pi \mathrm{i} \frac{\omega_{2}}{\omega_{1}}}, \quad \tilde{p}=e^{-2 \pi \mathrm{i} \frac{\omega_{2}}{\omega_{3}}}, \quad \tilde{r}=e^{-2 \pi \mathrm{i} \frac{\omega_{1}}{\omega_{3}}} .
$$

Modular parameters $\tau_{1}=\omega_{1} / \omega_{2}, \tau_{2}=\omega_{3} / \omega_{2}, \tau_{3}=\omega_{3} / \omega_{1}$ define three elliptic curves constrained by the condition $\tau_{3}=\tau_{2} / \tau_{1}$.

Elliptic gamma functions are defined as appropriate meromorphic solutions of the following finite difference equation

$$
f\left(u+\omega_{1}\right)=\theta\left(e^{2 \pi \mathrm{i} u / \omega_{2}} ; p\right) f(u), \quad u \in \mathbb{C} .
$$

Its particular solution, called the standard elliptic gamma function, has the form

$$
f(u)=\Gamma\left(e^{2 \pi \mathrm{i} u / \omega_{2}} ; p, q\right), \quad \Gamma(z ; p, q)=\prod_{j, k=0}^{\infty} \frac{1-z^{-1} p^{j+1} q^{k+1}}{1-z p^{j} q^{k}},
$$

where $|q|,|p|<1, z \in \mathbb{C}^{*}$ (note that the equation itself does not demand $|q|<1$ ). For incommensurate $\omega_{1,2,3}$, it can be defined uniquely as the meromorphic solution of (2.1) satisfying simultaneously two more equations:

$$
f\left(u+\omega_{2}\right)=f(u), \quad f\left(u+\omega_{3}\right)=\theta\left(e^{2 \pi \mathrm{i} u / \omega_{2}} ; q\right) f(u)
$$

and the normalization condition $f\left(\sum_{k=1}^{3} \omega_{k} / 2\right)=1$.

The modified elliptic gamma function has the form

$$
G(u ; \omega)=\Gamma\left(e^{2 \pi \mathrm{i} \frac{u}{\omega_{2}}} ; p, q\right) \Gamma\left(r e^{-2 \pi \mathrm{i} \frac{u}{\omega_{1}}} ; \tilde{q}, r\right) .
$$

It defines the unique simultaneous solution of equation (2.1) and two other equations:

$$
f\left(u+\omega_{2}\right)=\theta\left(e^{2 \pi \mathrm{i} u / \omega_{1}} ; r\right) f(u), \quad f\left(u+\omega_{3}\right)=\frac{\theta\left(e^{2 \pi \mathrm{i} \frac{u}{\omega_{2}}} ; q\right)}{\theta\left(e^{-2 \pi \mathrm{i} \frac{u}{\omega_{1}}} ; \tilde{q}\right)} f(u)
$$


with the same normalization condition $f\left(\sum_{k=1}^{3} \omega_{k} / 2\right)=1$. Here the third equation can be simplified using the modular transformation for theta functions

$$
\theta\left(e^{-2 \pi \mathrm{i} \frac{u}{\omega_{1}}} ; \tilde{q}\right)=e^{\pi \mathrm{i} B_{2,2}\left(u \mid \omega_{1}, \omega_{2}\right)} \theta\left(e^{2 \pi \mathrm{i} \frac{u}{\omega_{2}}} ; q\right),
$$

where

$$
B_{2,2}\left(u \mid \omega_{1}, \omega_{2}\right)=\frac{1}{\omega_{1} \omega_{2}}\left(u^{2}-\left(\omega_{1}+\omega_{2}\right) u+\frac{\omega_{1}^{2}+\omega_{2}^{2}}{6}+\frac{\omega_{1} \omega_{2}}{2}\right)
$$

is the second Bernoulli polynomial. These statements are based on the Jacobi theorem stating that if a meromorphic $\varphi(u)$ satisfies the system of equations

$$
\varphi\left(u+\omega_{1}\right)=\varphi\left(u+\omega_{2}\right)=\varphi\left(u+\omega_{3}\right)=\varphi(u)
$$

for $\omega_{1,2,3} \in \mathbb{C}$ linearly independent over $\mathbb{Z}$, then $\varphi(u)=$ const. The restricted values of bases $p^{n}=q^{m}, n, m \in \mathbb{Z}$ (or, equivalently, $r^{n}=\tilde{q}^{m}$ or $\tilde{r}^{n}=\tilde{p}^{m}$ ) may be called the torsion points, since the Jacobi theorem fails for them.

The function

$$
G(u ; \omega)=e^{-\frac{\pi \mathrm{i}}{3} B_{3,3}(u \mid \omega)} \Gamma\left(e^{-2 \pi \mathrm{i} \frac{u}{\omega_{3}}} ; \tilde{r}, \tilde{p}\right),
$$

where $|\tilde{p}|,|\tilde{r}|<1$ and

$$
\begin{aligned}
B_{3,3}\left(u \mid \omega_{1}, \omega_{2}, \omega_{3}\right) & =\frac{1}{\omega_{1} \omega_{2} \omega_{3}}\left(u^{3}-\frac{3 u^{2}}{2} \sum_{k=1}^{3} \omega_{k}\right. \\
+ & \left.\frac{u}{2}\left(\sum_{k=1}^{3} \omega_{k}^{2}+3 \sum_{1 \leq j<k \leq 3} \omega_{j} \omega_{k}\right)-\frac{1}{4}\left(\sum_{k=1}^{3} \omega_{k}\right) \sum_{1 \leq j<k \leq 3} \omega_{j} \omega_{k}\right)
\end{aligned}
$$

is the third Bernoulli polynomial, satisfies the same three equations and normalization as function (2.3). Hence they coincide, and this fact yields one of the $S L(3 ; \mathbb{Z})$-group modular transformation laws for the elliptic gamma function. From the expression (2.5) it is easy to see that $G(u ; \omega)$ is a meromorphic function of $u$ for $\omega_{1} / \omega_{2}>0$, i.e. when $|q|=1$. The region $|q|>1$ is similar to $|q|<1$, it can be reached by a symmetry transformation.

The theory of generalized gamma functions was built by Barnes [2]. Implicitly, the function $\Gamma(z ; p, q)$ appeared in the free energy per site of Baxter's eight vertex model [3] (see also [96] and [28]) - exactly in the form which will be used below in the superconformal indices context. A systematic investigation of its properties was launched by Ruijsenaars in [74]. Its $S L(3, \mathbb{Z})$ group transformation properties were described in [28]. The modified ("unit circle") elliptic gamma function $G(u ; \omega)$ was introduced in [83] (see also [21]). Both elliptic gamma functions are directly related to the Barnes multiple gamma function of the third order [31, 83].

In terms of the $\Gamma(z ; p, q)$-function one can write

$$
\theta(x ; p ; q)_{n}=\frac{\Gamma\left(x q^{n} ; p, q\right)}{\Gamma(x ; p, q)} .
$$

The short-hand conventions

$$
\begin{aligned}
& \Gamma\left(t_{1}, \ldots, t_{k} ; p, q\right):=\Gamma\left(t_{1} ; p, q\right) \cdots \Gamma\left(t_{k} ; p, q\right) \\
& \Gamma\left(t z^{ \pm 1} ; p, q\right):=\Gamma(t z ; p, q) \Gamma\left(t z^{-1} ; p, q\right), \quad \Gamma\left(z^{ \pm 2} ; p, q\right):=\Gamma\left(z^{2} ; p, q\right) \Gamma\left(z^{-2} ; p, q\right)
\end{aligned}
$$

are used below. The simplest properties of $\Gamma(z ; p, q)$ are:

- the symmetry $\Gamma(z ; p, q)=\Gamma(z ; q, p)$,

- the finite difference equations of the first order

$$
\Gamma(q z ; p, q)=\theta(z ; p) \Gamma(z ; p, q), \quad \Gamma(p z ; p, q)=\theta(z ; q) \Gamma(z ; p, q),
$$


- the reflection equation

$$
\Gamma(z ; p, q) \Gamma(p q / z ; p, q)=1
$$

- the duplication formula

$$
\Gamma\left(z^{2} ; p, q\right)=\Gamma\left(z,-z, q^{1 / 2} z,-q^{1 / 2} z, p^{1 / 2} z,-p^{1 / 2} z,(p q)^{1 / 2} z,-(p q)^{1 / 2} z ; p, q\right),
$$

- the limiting relations

$$
\lim _{p \rightarrow 0} \Gamma(z ; p, q)=\frac{1}{(z ; q)_{\infty}}, \quad \lim _{z \rightarrow 1}(1-z) \Gamma(z ; p, q)=\frac{1}{(p ; p)_{\infty}(q ; q)_{\infty}} .
$$

Definition 1. [82, 90] A meromorphic function $f\left(x_{1}, \ldots, x_{n} ; p\right)$ of $n$ variables $x_{j} \in \mathbb{C}^{*}$, which together with $p \in \mathbb{C}$ compose all indeterminates of this function, is called totally $p$-elliptic if

$$
f\left(p x_{1}, \ldots, x_{n} ; p\right)=\ldots=f\left(x_{1}, \ldots, p x_{n} ; p\right)=f\left(x_{1}, \ldots, x_{n} ; p\right),
$$

and if its divisor forms a nontrivial manifold of the maximal possible dimension.

Note that here positions of zeros and poles of elliptic functions are considered as indeterminates (i.e., they are not fixed in advance).

Consider $n$-dimensional integrals

$$
I\left(y_{1}, \ldots, y_{m}\right)=\int_{x \in D} \Delta\left(x_{1}, \ldots, x_{n} ; y_{1}, \ldots, y_{m}\right) \prod_{j=1}^{n} \frac{d x_{j}}{x_{j}},
$$

where $D \subset \mathbb{C}^{n}$ is some domain of integration and $\Delta\left(x_{1}, \ldots, x_{n} ; y_{1}, \ldots, y_{m}\right)$ is a meromorphic function of $x_{j}$ and $y_{k}$, where $y_{k}$ denote the "external" parameters.

Definition 2. [83] The integral $I\left(y_{1}, \ldots, y_{m} ; p, q\right)$ is called the elliptic hypergeometric integral if there are two distinguished complex parameters $p$ and $q$ such that I's kernel $\Delta\left(x_{1}, \ldots\right.$, $\left.x_{n} ; y_{1}, \ldots, y_{m} ; p, q\right)$ satisfies the following system of linear first order $q$-difference equations in the integration variables $x_{j}$ :

$$
\frac{\Delta\left(\ldots q x_{j} \ldots ; y_{1}, \ldots, y_{m} ; p, q\right)}{\Delta\left(x_{1}, \ldots, x_{n} ; y_{1}, \ldots, y_{m} ; p, q\right)}=h_{j}\left(x_{1}, \ldots, x_{n} ; y_{1}, \ldots, y_{m} ; q ; p\right),
$$

where $h_{j}$ are some p-elliptic functions of the variables $x_{j}$,

$$
h_{j}\left(\ldots p x_{i} \ldots ; y_{1}, \ldots, y_{m} ; q ; p\right)=h_{j}\left(x_{1}, \ldots, x_{n} ; y_{1}, \ldots, y_{m} ; q ; p\right) .
$$

The kernel $\Delta$ is called then the elliptic hypergeometric term, and the functions $h_{j}\left(x_{1}, \ldots\right.$, $\left.x_{n} ; y_{1}, \ldots, y_{m} ; q ; p\right)$ - the $q$-certificates.

This definition is not the most general possible one of such kind, but it is sufficient for the purposes of the present paper. The elliptic hypergeometric series can be introduced as sums of residues of particular sequences of poles of the elliptic hypergeometric integral kernels [19] and, because of the convergence difficulties, they are less general than the integrals. In the onedimensional case, $n=1$, the structure of admissible elliptic hypergeometric terms $\Delta$ can be described explicitly. Indeed, any meromorphic $p$-elliptic function $f(p x)=f(x)$ can be written in the form

$$
f_{p}(x)=z \prod_{k=1}^{N} \frac{\theta\left(t_{k} x ; p\right)}{\theta\left(w_{k} x ; p\right)}, \quad \prod_{k=1}^{N} t_{k}=\prod_{k=1}^{N} w_{k},
$$


where $z, t_{1}, \ldots, t_{N}, w_{1}, \ldots, w_{N}$ are arbitrary complex parameters. The positive integer $N$ is called the order of the elliptic function, and the linear constraint on parameters - the balancing condition. From the identity

$$
z=\frac{\theta(z x, p x ; p)}{\theta(p z x, x ; p)}
$$

we see that $z$ is not a distinguished parameter - it can be obtained from $t_{k}$ and $w_{k}$ by appropriate reduction without spoiling the balancing condition. Therefore we set $z=1$.

Now, for $|q|<1$, the general solution of the equation $\Delta(q x)=f_{p}(x) \Delta(x)$ is

$$
\Delta(x)=\varphi(x) \prod_{k=1}^{N} \frac{\Gamma\left(t_{k} x ; p, q\right)}{\Gamma\left(w_{k} x ; p, q\right)}, \quad \varphi(x)=\prod_{k=1}^{M} \frac{\theta\left(a_{k} x ; q\right)}{\theta\left(b_{k} x ; q\right)}, \quad \prod_{k=1}^{M} a_{k}=\prod_{k=1}^{M} b_{k},
$$

where $\varphi(q x)=\varphi(x)$ is an arbitrary $q$-elliptic function. However, since

$$
\varphi(x)=\prod_{k=1}^{M} \frac{\Gamma\left(p a_{k} x, b_{k} x ; p, q\right)}{\Gamma\left(a_{k} x, p b_{k} x ; p, q\right)}
$$

one can obtain $\varphi(x)$ from ratios of $\Gamma$-functions after replacing $N$ by $N+2 M$ and appropriate specification of the original parameters $t_{k}$ and $w_{k}$ with the balancing condition preserved. Therefore we can drop $\varphi(x)$ function and find that the general elliptic hypergeometric term for $n=1$ has the form:

$$
\Delta\left(x ; t_{1}, \ldots, t_{N}, w_{1}, \ldots, w_{N} ; p, q\right)=\prod_{k=1}^{N} \frac{\Gamma\left(t_{k} x ; p, q\right)}{\Gamma\left(w_{k} x ; p, q\right)}, \quad \prod_{k=1}^{N} \frac{t_{k}}{w_{k}}=1 .
$$

This function is symmetric in $p$ and $q$, i.e. we can repeat the above considerations with these parameters permuted. Then, for incommensurate $p$ and $q$ (i.e., when $p^{j} \neq q^{k}, j, k \in \mathbb{Z}$ ), the equations

$$
\Delta(q x)=f_{p}(x) \Delta(x), \quad \Delta(p x)=f_{q}(x) \Delta(x)
$$

determine $\Delta(x)$ uniquely up to a multiplicative constant.

For $|q|>1$,

$$
\Delta\left(x ; t_{1}, \ldots, t_{N}, w_{1}, \ldots, w_{N} ; p, q\right)=\prod_{k=1}^{N} \frac{\Gamma\left(q^{-1} w_{k} x ; p, q^{-1}\right)}{\Gamma\left(q^{-1} t_{k} x ; p, q^{-1}\right)}, \quad \prod_{k=1}^{N} \frac{t_{k}}{w_{k}}=1 .
$$

For $|q|=1$, the requirement of meromorphicity in $x$ is too strong. To define integrals in this case one has to use the modified elliptic gamma function $G(u ; \omega)$, or modular transformations, which we skip for brevity.

In analogy with the series case considered in [82], it is natural to extend the notion of total ellipticity to elliptic hypergeometric terms entering integrals [83].

Definition 3. An elliptic hypergeometric integral

$$
I\left(y_{1}, \ldots, y_{m} ; p, q\right)=\int_{x \in D} \Delta\left(x_{1}, \ldots, x_{n} ; y_{1}, \ldots, y_{m} ; p, q\right) \prod_{j=1}^{n} \frac{d x_{j}}{x_{j}}
$$

is called totally elliptic if all its kernel's $q$-certificates $h_{j}\left(x_{1}, \ldots, x_{n} ; y_{1}, \ldots, y_{m} ; q ; p\right), j=1, \ldots, n+$ $m$, are totally elliptic functions, $i . e$. they are $p$-elliptic in all variables $x_{1}, \ldots, x_{n}, y_{1}, \ldots, y_{m}$ and q. In particular,

$$
h_{j}\left(x_{1}, \ldots, x_{n} ; y_{1}, \ldots, y_{m} ; p q ; p\right)=h_{j}\left(x_{1}, \ldots, x_{n} ; y_{1}, \ldots, y_{m} ; q ; p\right) .
$$


Theorem 1 (Rains, Spiridonov, 2004). Given $\mathbb{Z}^{n} \rightarrow \mathbb{Z}$ maps $\epsilon\left(m^{(a)}\right)=\epsilon\left(m_{1}^{(a)}, \ldots, m_{n}^{(a)}\right)$, $a=1, \ldots, M$, with finite support, define the meromorphic function

$$
\Delta\left(x_{1}, \ldots, x_{n} ; p, q\right)=\prod_{a=1}^{M} \Gamma\left(x_{1}^{m_{1}^{(a)}} x_{2}^{m_{2}^{(a)}} \ldots x_{n}^{m_{n}^{(a)}} ; p, q\right)^{\epsilon\left(m^{(a)}\right)} .
$$

Suppose $\Delta$ is a totally elliptic hypergeometric term, i.e. its q-certificates are p-elliptic functions of $q$ and $x_{1}, \ldots, x_{n}$. Then these certificates are also modular invariant.

The proof is elementary. The $q$-certificates have the explicit form

$$
h_{i}(x ; q ; p)=\frac{\Delta\left(\ldots q x_{i} \ldots ; p, q\right)}{\Delta\left(x_{1}, \ldots, x_{n} ; p, q\right)}=\prod_{a=1}^{M} \theta\left(x^{m^{(a)}} ; p ; q\right)_{m_{i}^{(a)}}^{\epsilon\left(m^{(a)}\right)} .
$$

The conditions for $h_{i}$ to be elliptic in $x_{j}$ yield the constraints

$$
\begin{aligned}
& \sum_{a=1}^{M} \epsilon\left(m^{(a)}\right) m_{i}^{(a)} m_{j}^{(a)} m_{k}^{(a)}=0 \\
& \sum_{a=1}^{M} \epsilon\left(m^{(a)}\right) m_{i}^{(a)} m_{j}^{(a)}=0
\end{aligned}
$$

for $1 \leq i, j, k \leq n$. The conditions of ellipticity in $q$ add one more constraint

$$
\sum_{a=1}^{M} \epsilon\left(m^{(a)}\right) m_{i}^{(a)}=0 .
$$

The latter equation guarantees that $h_{i}$ has an equal number of theta functions in its numerator and denominator. The modular invariance of $h_{i}$ follows then automatically from the transformation property (2.4). Such a direct relation between total ellipticity and modularity was conjectured to be true in general in [82].

The simplest known nontrivial totally elliptic hypergeometric term corresponds to $n=$ 6, $M=29$ and has the form [86]

$$
\Delta\left(x ; t_{1}, \ldots, t_{5} ; p, q\right)=\frac{\prod_{j=1}^{5} \Gamma\left(t_{j} x^{ \pm 1}, t_{j}^{-1} \prod_{i=1}^{5} t_{i} ; p, q\right)}{\Gamma\left(x^{ \pm 2}, \prod_{i=1}^{5} t_{i} x^{ \pm 1} ; p, q\right) \prod_{1 \leq i<j \leq 5} \Gamma\left(t_{i} t_{j} ; p, q\right)} .
$$

Theorem 2. [81] Elliptic beta integral. For $|p|,|q|,\left|t_{j}\right|<1,\left|\prod_{j=1}^{5} t_{j}\right|<|p q|$, one has

$$
\frac{(p ; p)_{\infty}(q ; q)_{\infty}}{4 \pi \mathrm{i}} \int_{\mathbb{T}} \Delta\left(x ; t_{1}, \ldots, t_{5} ; p, q\right) \frac{d x}{x}=1,
$$

where $\mathbb{T}$ is the unit circle with positive orientation.

The Euler beta integral evaluation formula (1.1) lies at the bottom of this identity. On the corresponding degeneration road one finds many interesting integrals, including the Rahman and Askey-Wilson q-beta integrals [1]. Formula (2.10) served as an entry ticket to the large class of new exactly computable integrals discussed in $[19,20,21,65,83,93]$, which is essentially extended by the conjectures presented in this paper. In $[83,85,87]$ the elliptic beta integral was generalized to an elliptic analogue of the Gauss hypergeometric function obeying many classical properties. For a survey of this function and its generalizations to higher order elliptic hypergeometric functions and multiple integrals on root systems, see [89].

Two totally elliptic hypergeometric terms associated with the elliptic beta integrals of type I on root systems $B C_{n}$ [20] and $A_{n}$ [83] were constructed in [86]. One more similar example 
for the root system $A_{n}$ was built in [93]. Some time ago, using the combination of techniques introduced in [86] and [69], the first author has further generalized the former two terms to an arbitrary number of parameters [90]. For instance, define the kernel

$$
\Delta_{n}(z, t ; p, q)=\prod_{1 \leq i<j \leq n} \frac{1}{\Gamma\left(z_{i}^{ \pm 1} z_{j}^{ \pm 1} ; p, q\right)} \prod_{j=1}^{n} \frac{\prod_{i=1}^{2 n+2 m+4} \Gamma\left(t_{i} z_{j}^{ \pm 1} ; p, q\right)}{\Gamma\left(z_{j}^{ \pm 2} ; p, q\right)}
$$

and the type I $B C_{n}$-elliptic hypergeometric integral:

$$
I_{n}^{(m)}\left(t_{1}, \ldots, t_{2 n+2 m+4}\right)=\frac{(p ; p)_{\infty}^{n}(q ; q)_{\infty}^{n}}{2^{n} n !(2 \pi \mathrm{i})^{n}} \int_{\mathbb{T}^{n}} \Delta_{n}(z, t ; p, q) \prod_{j=1}^{n} \frac{d z_{j}}{z_{j}}
$$

where $\left|t_{j}\right|<1$ and $\prod_{j=1}^{2 n+2 m+4} t_{j}=(p q)^{m+1}$.

Theorem 3. [65] For $|p q|^{1 / 2}<\left|t_{j}\right|<1$, the integrals $I_{n}^{(m)}$ satisfy the relation

$$
I_{n}^{(m)}\left(t_{1}, \ldots, t_{2 n+2 m+4}\right)=\prod_{1 \leq r<s \leq 2 n+2 m+4} \Gamma\left(t_{r} t_{s} ; p, q\right) I_{m}^{(n)}\left(\frac{\sqrt{p q}}{t_{1}}, \ldots, \frac{\sqrt{p q}}{t_{2 n+2 m+4}}\right) .
$$

This is an elliptic analogue of the symmetry transformation for some plain hypergeometric integrals established by Dixon in [23].

Theorem 4. [90] The ratio

$$
\rho(z, y ; t ; p, q)=\prod_{1 \leq r<s \leq 2 n+2 m+4} \Gamma\left(t_{r} t_{s} ; p, q\right)^{-1} \frac{\Delta_{n}(z ; t ; p, q)}{\Delta_{m}(y / \sqrt{p q} ; \sqrt{p q} / t ; p, q)}
$$

is the totally elliptic hypergeometric term. That is all ratios $\rho(\ldots, q v, \ldots) / \rho(\ldots, v, \ldots)$ for $v \in\left\{z_{1}, \ldots, z_{n}, y_{1}, \ldots, y_{m}, t_{1}, \ldots, t_{2 n+2 m+4}\right\}$ are $p$-elliptic functions of all variables $z_{i}, y_{k}, t_{l}$, and $q$.

The term $\rho(z, y ; t ; p, q)$ contains elliptic gamma functions with non-removable integer powers of $p q$ in the argument. Therefore the ansatz (2.6) does not cover all interesting totally elliptic hypergeometric terms. As we shall show below, there are also examples of terms depending on fractional powers of $p q$. For them the total ellipticity condition is slightly modified: it is necessary to consider dilations of the parameter $q$ by appropriate powers of $p$. Introducing the variable $x_{0}=(p q)^{1 / K}, K=1,2, \ldots$ and adding to the arguments of elliptic gamma functions in (2.6) the multipliers $x_{0}^{m_{0}^{(a)}}$, it is not difficult to find the general form of constraints on integers $m_{j}^{(a)}$ and $\epsilon\left(m^{(a)}\right)$ guaranteeing total ellipticity (with special $p^{K}$-ellipticity condition for the variable $q$ ). However, these constraints look essentially less beautiful than the Diophantine equations described above. Moreover, at the moment it is not clear which part of the modular transformation group survives because of the presence of fractional parts of modular variables in the arguments of respective elliptic functions-certificates.

In the present work, we have checked that all nontrivial relations for elliptic hypergeometric integrals described below define totally elliptic hypergeometric terms through the ratios of the corresponding integral kernels. Namely, this property was verified for the equalities of superconformal indices in

- the initial Seiberg duality (4.6) and (4.7); SP-groups duality (5.1) and (5.2); [90]

- multiple dualities for $S P(2 N)$ gauge group (6.1), (6.2), (6.3) and (6.4);

- new duality for $S P(2 N)$ group (7.1) and (7.2);

- multiple duality for $S U(2 N)$ gauge group (8.1), (8.2), (8.3) and (8.4); 
- KS type dualities for unitary groups (9.2) and (9.3) (see Appendix D for a detailed consideration of this case); (9.5) and (9.6); (9.8) and (9.9); (9.10) and (9.11); (9.13) and (9.14); (9.16) and (9.17); (9.19) and (9.20); (9.22) and (9.23);

- KS type dualities for symplectic groups (10.2) and (10.3); (10.5) and (10.6); (10.8) and (10.9); (10.11) and (10.12);

- confinement for $S U(N)$ group theories (12.1) and (12.2); (12.3) and (12.4); (12.5) and (12.6); (12.7) and (12.8); (12.9) and (12.10); (12.19) and (12.20); (12.21) and (12.22); (12.25) and (12.26); (12.27) and (12.28); (12.29) and (12.30); (12.31) and (12.32);

- confinement for $S P(2 N)$ group theories (12.33) and (12.34); (12.35) and (12.36); (12.37) and (12.38);

- dualities for the $G_{2}$ gauge group (13.1) and (13.2); (13.3) and (13.4).

Our auxiliary file with the details of these verifications is bigger than the present paper. During this work we have found a number of mistakes in the description of hypercharges of the fields in some original papers. On the basis of this large amount of computations, we put forward the following conjecture.

Conjecture. The condition of total ellipticity for the elliptic hypergeometric terms is necessary for the existence of the exact integration formulas for elliptic beta integrals or of the nontrivial Weyl group symmetry transformations for the elliptic hypergeometric integrals.

It is known that behind each elliptic hypergeometric integral there is a terminating elliptic hypergeometric series appearing from the residue calculus for restricted values of parameters [19]. The above conjecture has a natural meaning in terms of such series - it simply demands that the summation or transformation identities for them involve ratios of Jacobi forms with appropriate quasiperiodicity and modularity properties in the sense of Eichler and Zagier [27]. Already this fact is sufficient (when there are no fractional powers of $p q$ ) for the confirmation of the series identities to rather high powers of small $\log q$ expansions [19].

For a given elliptic hypergeometric integral there may exist more than one totally elliptic hypergeometric term. For the terms associated with elliptic beta integrals discussed in [86, 93] there existed a complementary difference equation with the totally elliptic function coefficients. During our work we have found examples of fake terms which do not lead to identities (or fake anomaly matching conditions without real duality). Therefore analysis of the sufficiency condition for existence of nontrivial identities looks much more neat - it should address the nonuniqueness questions and the list of additional admissible technical tools. Sometimes the ratio of a given elliptic hypergeometric integral kernel to itself with different integration variables yields the totally elliptic hypergeometric term. It may happen that for a fixed set of parameters, it is sufficient to have totally elliptic hypergeometric terms of a more complicated nature than the latter one, and then at least one of them will lead to a nontrivial relation between integrals.

\section{SUPERCONFORMAL INDEX}

3.1. $\mathcal{N}=1$ superconformal algebra. In 4 dimensional space-time the conformal algebra $S O(4,2)$ is formed by the generators of translations $P_{a}$, special conformal transformations $K_{a}, S O(3,1)$ Lorentz group rotations, $M_{a b}=-M_{b a}$, and the dilations $H$. The commutation relations have the form [25]

$$
\begin{aligned}
& {\left[M_{a b}, P_{c}\right]=i\left(\eta_{a c} P_{b}-\eta_{b c} P_{a}\right), \quad\left[M_{a b}, K_{c}\right]=i\left(\eta_{a c} K_{b}-\eta_{b c} K_{a}\right),} \\
& {\left[M_{a b}, M_{c d}\right]=i\left(\eta_{a c} M_{b d}-\eta_{b c} M_{a d}-\eta_{a d} M_{b c}+\eta_{b d} M_{a c}\right),} \\
& {\left[H, P_{a}\right]=P_{a}, \quad\left[H, K_{a}\right]=-K_{a}, \quad\left[K_{a}, P_{b}\right]=-2 i M_{a b}-2 \eta_{a b} H,}
\end{aligned}
$$


ELLIPTIC HYPERGEOMETRY OF SUPERSYMMETRIC DUALITIES

where $\eta_{a b}=\operatorname{diag}(-1,1,1,1)$ and all indices take values $a=0,1,2,3$. In terms of the matrix

$$
M_{A B}=\left(\begin{array}{ccc}
M_{a b} & -\frac{1}{2}\left(P_{a}-K_{a}\right) & -\frac{1}{2}\left(P_{a}+K_{a}\right) \\
\frac{1}{2}\left(P_{b}-K_{b}\right) & 0 & i H \\
\frac{1}{2}\left(P_{b}+K_{b}\right) & -i H & 0
\end{array}\right)
$$

where $A, B=0, \ldots, 5$, relations (3.1) are rewritten in the simpler form [25]

$$
\left[M_{A B}, M_{C D}\right]=i\left(\eta_{A C} M_{B D}-\eta_{B C} M_{A D}-\eta_{A D} M_{B C}+\eta_{B D} M_{A C}\right)
$$

with $\eta_{A B}=\operatorname{diag}(-1,1,1,1,1,-1)$.

In the spinorial basis one defines

$$
\begin{array}{ll}
P_{\alpha \dot{\alpha}}=\left(\sigma^{a}\right)_{\alpha \dot{\alpha}} P_{a}, & K^{\dot{\alpha} \alpha}=\left(\bar{\sigma}^{a}\right)^{\dot{\alpha} \alpha} K_{a}, \\
M_{\alpha}^{\beta}=-\frac{i}{4}\left(\sigma^{a} \bar{\sigma}^{b}\right)_{\alpha}^{\beta} M_{a b}, & \bar{M}_{\dot{\beta}}^{\dot{\alpha}}=-\frac{i}{4}\left(\bar{\sigma}^{a} \sigma^{b}\right)_{\dot{\beta}}^{\dot{\alpha}} M_{a b},
\end{array}
$$

where $\alpha, \dot{\alpha}, \beta, \dot{\beta}=1,2$,

$$
\sigma^{a}=\left(I, \sigma^{i}\right), \quad \bar{\sigma}^{a}=\left(I,-\sigma^{i}\right)
$$

and $\sigma^{i}$ are the usual Pauli matrices

$$
\sigma^{1}=\left(\begin{array}{ll}
0 & 1 \\
1 & 0
\end{array}\right), \quad \sigma^{2}=\left(\begin{array}{cc}
0 & -i \\
i & 0
\end{array}\right), \quad \sigma^{3}=\left(\begin{array}{cc}
1 & 0 \\
0 & -1
\end{array}\right) .
$$

Using the standard angular momentum generators, we set

$$
M_{\alpha}^{\beta}=\left(\begin{array}{cc}
J_{3} & J_{+} \\
J_{-} & -J_{3}
\end{array}\right), \quad \bar{M}_{\dot{\beta}}^{\dot{\alpha}}=\left(\begin{array}{cc}
\bar{J}_{3} & \bar{J}_{+} \\
\bar{J}_{-} & -\bar{J}_{3}
\end{array}\right),
$$

with $\left[J_{3}, J_{ \pm}\right]= \pm J_{ \pm},\left[J_{+}, J_{-}\right]=2 J_{3}$ and similar relations for $\bar{J}_{ \pm}, \bar{J}_{3}$. Then the tensor $M_{a b}$ is expressed through these operators as

$$
M_{a b}=\left(\begin{array}{cccc}
0 & \frac{i}{2}\left(\bar{J}_{+}+\bar{J}_{-}-J_{+}-J_{-}\right) & \frac{1}{2}\left(J_{+}+\bar{J}_{-}-\bar{J}_{+}-J_{-}\right) & i\left(\bar{J}_{3}-J_{3}\right) \\
-\frac{i}{2}\left(\bar{J}_{+}+\bar{J}_{-}-J_{+}-J_{-}\right) & 0 & -\left(J_{3}+\bar{J}_{3}\right) & \frac{i}{2}\left(J_{+}+\bar{J}_{+}-J_{-}-\bar{J}_{-}\right) \\
-\frac{1}{2}\left(J_{+}+\bar{J}_{-}-\bar{J}_{+}-J_{-}\right) & \left(J_{3}+\bar{J}_{3}\right) & 0 & -\frac{1}{2}\left(J_{+}+J_{-}+\bar{J}_{+}+\bar{J}_{-}\right) \\
-i\left(\bar{J}_{3}-J_{3}\right) & -\frac{i}{2}\left(J_{+}+\bar{J}_{+}-J_{-}-\bar{J}_{-}\right) & \frac{1}{2}\left(J_{+}+J_{-}+\bar{J}_{+}+\bar{J}_{-}\right) & 0
\end{array}\right) .
$$

The conformal algebra (3.1) can be rewritten now as

$$
\begin{array}{ll}
{\left[M_{\alpha}{ }^{\beta}, M_{\gamma}{ }^{\delta}\right]=\delta_{\gamma}^{\beta} M_{\alpha}{ }^{\delta}-\delta_{\alpha}^{\delta} M_{\gamma}^{\beta},} & {\left[\bar{M}_{\dot{\beta}}^{\dot{\alpha}}, \bar{M}_{\dot{\delta}}^{\dot{\gamma}}\right]=\delta_{\dot{\beta}}^{\dot{\gamma}} \bar{M}_{\dot{\delta}}^{\dot{\alpha}}-\delta_{\dot{\delta}}^{\dot{\alpha}} \bar{M}_{\dot{\beta}}^{\dot{\gamma}},} \\
{\left[M_{\alpha}{ }^{\beta}, P_{\gamma \dot{\delta}}\right]=\delta_{\gamma}^{\beta} P_{\alpha \dot{\delta}}-\frac{1}{2} \delta_{\alpha}^{\beta} P_{\gamma \dot{\delta}},} & {\left[\bar{M}_{\dot{\beta}}^{\dot{\alpha}}, P_{\gamma \dot{\delta}}\right]=-\delta_{\dot{\delta}}^{\dot{\alpha}} P_{\gamma \dot{\beta}}+\frac{1}{2} \delta_{\dot{\beta}}^{\dot{\alpha}} P_{\gamma \dot{\delta}},} \\
{\left[M_{\alpha}{ }^{\beta}, K^{\dot{\gamma} \delta}\right]=-\delta_{\alpha}^{\delta} K^{\dot{\gamma} \beta}+\frac{1}{2} \delta_{\alpha}^{\beta} K^{\dot{\gamma} \delta},} & {\left[\bar{M}_{\dot{\beta}}^{\dot{\alpha}}, K^{\dot{\gamma} \delta}\right]=\delta_{\dot{\beta}}^{\dot{\gamma}} K^{\dot{\alpha} \delta}-\frac{1}{2} \delta_{\dot{\beta}}^{\dot{\alpha}} K^{\dot{\gamma} \delta},} \\
{\left[M_{\alpha}{ }^{\beta}, H\right]=0,} & {\left[\bar{M}_{\dot{\beta}}^{\dot{\alpha}}, H\right]=0,} \\
{\left[H, P_{\alpha \dot{\beta}}\right]=P_{\alpha \dot{\beta}},} & {\left[H, K^{\dot{\alpha} \beta}\right]=-K^{\dot{\alpha} \beta},} \\
{\left[P_{\alpha \dot{\beta}}, K^{\dot{\gamma} \delta}\right]=4\left(\delta_{\dot{\beta}}^{\dot{\gamma}} M_{\alpha}^{\delta}-\delta_{\alpha}^{\delta} \bar{M}_{\dot{\beta}}^{\dot{\gamma}}+\delta_{\dot{\beta}}^{\dot{\gamma}} \delta_{\alpha}^{\delta} H\right) .}
\end{array}
$$

$S O(4,2)$ (or $S U(2,2)$ ) algebra can be extended by adding supercharges $Q_{\alpha}, \bar{Q}_{\dot{\alpha}}$ and their superconformal partners $S^{\alpha}, \bar{S}^{\dot{\alpha}}$. Supercharges satisfy the anticommutator relations [25, 97]

$$
\left\{Q_{\alpha}, \bar{Q}_{\dot{\alpha}}\right\}=2 P_{\alpha \dot{\alpha}}, \quad\left\{Q_{\alpha}, Q_{\beta}\right\}=\left\{\bar{Q}_{\dot{\alpha}}, \bar{Q}_{\dot{\beta}}\right\}=0,
$$

while their superconformal partners obey

$$
\left\{\bar{S}^{\dot{\alpha}}, S^{\alpha}\right\}=2 K^{\dot{\alpha} \alpha}, \quad\left\{\bar{S}^{\dot{\alpha}}, \bar{S}^{\dot{\beta}}\right\}=\left\{S^{\alpha}, S^{\beta}\right\}=0 .
$$


The cross-anti-commutators of $Q_{\alpha}$ and $S_{\alpha}$ have the form

$$
\left\{Q_{\alpha}, \bar{S}^{\dot{\alpha}}\right\}=0, \quad\left\{S^{\alpha}, \bar{Q}_{\dot{\alpha}}\right\}=0,
$$

while

$$
\begin{aligned}
& \left\{Q_{\alpha}, S^{\beta}\right\}=4\left(M_{\alpha}^{\beta}+\frac{1}{2} \delta_{\alpha}^{\beta} H+\frac{3}{4} \delta_{\alpha}^{\beta} R\right), \\
& \left\{\bar{S}^{\dot{\alpha}}, \bar{Q}_{\dot{\beta}}\right\}=4\left(\bar{M}_{\dot{\beta}}^{\dot{\alpha}}-\frac{1}{2} \delta_{\dot{\beta}}^{\dot{\alpha}} H+\frac{3}{4} \delta_{\dot{\beta}}^{\dot{\alpha}} R\right),
\end{aligned}
$$

where $R$ is the $R$-charge generating $U(1)_{R}$-symmetry group.

The bosonic and fermionic generators cross-commute as

$$
\begin{aligned}
& {\left[M_{\alpha}^{\beta}, Q_{\gamma}\right]=\delta_{\gamma}^{\beta} Q_{\alpha}-\frac{1}{2} \delta_{\alpha}^{\beta} Q_{\gamma}, \quad\left[M_{\alpha}^{\beta}, \bar{Q}_{\dot{\gamma}}\right]=0,} \\
& {\left[M_{\alpha}^{\beta}, S^{\gamma}\right]=-\delta_{\alpha}^{\gamma} S^{\beta}+\frac{1}{2} \delta_{\alpha}^{\beta} S^{\gamma}, \quad\left[M_{\alpha}^{\beta}, \bar{S}^{\dot{\gamma}}\right]=0,} \\
& {\left[\bar{M}_{\beta}^{\dot{\alpha}}, Q_{\gamma}\right]=0, \quad\left[\bar{M}_{\dot{\beta}}^{\dot{\alpha}}, \bar{Q}_{\dot{\gamma}}\right]=-\delta_{\dot{\gamma}}^{\dot{\alpha}} \bar{Q}_{\dot{\beta}}+\frac{1}{2} \delta_{\dot{\beta}}^{\dot{\alpha}} \bar{Q}_{\dot{\gamma}},} \\
& {\left[\bar{M}_{\beta}^{\dot{\alpha}}, S^{\gamma}\right]=0, \quad\left[\bar{M}_{\dot{\beta}}^{\dot{\alpha}}, \bar{S}^{\dot{\gamma}}\right]=\delta_{\dot{\beta}}^{\dot{\gamma}} \bar{S}^{\dot{\alpha}}-\frac{1}{2} \delta_{\dot{\beta}}^{\dot{\alpha}} \bar{S}^{\dot{\gamma}},} \\
& {\left[P_{\alpha \dot{\beta}}, S^{\gamma}\right]=\delta_{\alpha}^{\gamma} \bar{Q}_{\dot{\beta}}, \quad\left[P_{\alpha \dot{\beta}}, \bar{S}^{\dot{\gamma}}\right]=\delta_{\dot{\beta}}^{\dot{\gamma}} Q_{\alpha},} \\
& {\left[K^{\dot{\alpha} \beta}, Q_{\gamma}\right]=\delta_{\gamma}^{\beta} \bar{S}^{\dot{\alpha}}, \quad\left[K^{\dot{\alpha} \beta}, \bar{Q}_{\dot{\gamma}}\right]=\delta_{\dot{\gamma}}^{\dot{\alpha}} S^{\beta},} \\
& {\left[H, Q_{\alpha}\right]=\frac{1}{2} Q_{\alpha}, \quad\left[H, \bar{Q}_{\dot{\alpha}}\right]=\frac{1}{2} \bar{Q}_{\dot{\alpha}},} \\
& {\left[H, S^{\alpha}\right]=-\frac{1}{2} S^{\alpha},}
\end{aligned}
$$

The $R$-charge commutes with all bosonic generators and has non-trivial commutators only with the supercharges and their superconformal partners

$$
\begin{array}{lrl}
{\left[R, Q_{\alpha}\right]} & =-Q_{\alpha}, & {\left[R, \bar{Q}_{\dot{\alpha}}\right]=\bar{Q}_{\dot{\alpha}},} \\
{\left[R, S^{\alpha}\right]} & =S^{\alpha}, & {\left[R, \bar{S}^{\dot{\alpha}}\right]=-\bar{S}^{\dot{\alpha}} .}
\end{array}
$$

To simplify the shape of the $\mathcal{N}=1$ superconformal algebra one introduces the notations

$$
\mathcal{M}_{\mathcal{A}}^{\mathcal{B}}=\left(\begin{array}{cc}
M_{\alpha}^{\beta}+\frac{1}{2} \delta_{\alpha}^{\beta} H & \frac{1}{2} P_{\alpha \dot{\beta}} \\
\frac{1}{2} K^{\dot{\alpha} \beta} & \bar{M}_{\dot{\beta}}^{\dot{\alpha}}-\frac{1}{2} \delta_{\dot{\beta}}^{\dot{\alpha}} H
\end{array}\right), \quad \mathcal{Q}_{\mathcal{A}}=\left(\begin{array}{c}
Q_{\alpha} \\
\bar{S}^{\dot{\alpha}}
\end{array}\right), \quad \overline{\mathcal{Q}}^{\mathcal{B}}=\left(\begin{array}{ll}
S^{\beta} & \bar{Q}_{\dot{\beta}}
\end{array}\right)
$$

Then the (anti)commutators $(3.6),(3.7),(3.8),(3.9),(3.11),(3.12)$ combine to [26]

$$
\begin{aligned}
& {\left[\mathcal{M}_{\mathcal{A}}^{\mathcal{B}}, \mathcal{M}_{\mathcal{C}}^{\mathcal{D}}\right]=\delta_{\mathcal{C}}^{\mathcal{B}} \mathcal{M}_{\mathcal{A}}^{\mathcal{D}}-\delta_{\mathcal{A}}^{\mathcal{D}} \mathcal{M}_{\mathcal{C}}^{\mathcal{B}},} \\
& {\left[\mathcal{M}_{\mathcal{A}}^{\mathcal{B}}, \mathcal{Q}_{\mathcal{C}}\right]=\delta_{\mathcal{C}}^{\mathcal{B}} \mathcal{Q}_{\mathcal{A}}-\frac{1}{4} \delta_{\mathcal{A}}^{\mathcal{B}} \mathcal{Q}_{\mathcal{C}}, \quad\left[\mathcal{M}_{\mathcal{A}}^{\mathcal{B}}, \overline{\mathcal{Q}}^{\mathcal{C}}\right]=-\delta_{\mathcal{A}}^{\mathcal{C}} \overline{\mathcal{Q}}^{\mathcal{B}}+\frac{1}{4} \delta_{\mathcal{A}}^{\mathcal{B}} \overline{\mathcal{C}}^{\mathcal{C}},} \\
& {\left[R, \mathcal{Q}_{\mathcal{A}}\right]=-\mathcal{Q}_{A}, \quad\left[R, \overline{\mathcal{Q}}^{\mathcal{B}}\right]=\overline{\mathcal{Q}}^{\mathcal{B}},} \\
& \left\{\mathcal{Q}_{\mathcal{A}}, \overline{\mathcal{Q}}^{\mathcal{B}}\right\}=4 \mathcal{M}_{\mathcal{A}}^{\mathcal{B}}+3 \delta_{\mathcal{A}}^{\mathcal{B}} R, \quad\left\{\mathcal{Q}_{\mathcal{A}}, \mathcal{Q}_{\mathcal{B}}\right\}=0, \quad\left\{\overline{\mathcal{Q}}^{\mathcal{A}}, \overline{\mathcal{Q}}^{\mathcal{B}}\right\}=0,
\end{aligned}
$$

where

$$
\delta_{\mathcal{A}}^{\mathcal{B}}=\left(\begin{array}{cc}
\delta_{\alpha}^{\beta} & 0 \\
0 & \delta_{\dot{\beta}}^{\dot{\alpha}}
\end{array}\right)
$$


3.2. The index. Suppose an operator $Q$ and its Hermitian conjugate $Q^{\dagger}$ satisfy the relations

$$
\{Q, Q\}=0, \quad\left\{Q^{\dagger}, Q^{\dagger}\right\}=0, \quad\left\{Q, Q^{\dagger}\right\}=2 H,
$$

where $H$ is the Hamiltonian $\left(=P_{0}\right)$ of a taken system. This is a universal situation valid down to the non-relativistic quantum mechanics. The Witten index [99] defined as $\operatorname{Tr}(-1)^{\mathrm{F}}$ tells (under certain conditions) whether the supersymmetry is broken spontaneously or not. By definition the operator $(-1)^{\mathrm{F}}$ is

$$
(-1)^{\mathrm{F}}=\exp \left(2 \pi \mathrm{i} J_{z}\right), \quad\left\{Q,(-1)^{\mathrm{F}}\right\}=0,
$$

where in the spinorial basis $J_{z}=-J_{3}-\bar{J}_{3}$. It distinguishes bosonic states $|b\rangle$ from the fermionic ones $|f\rangle$,

$$
(-1)^{\mathrm{F}}|b\rangle=|b\rangle, \quad(-1)^{\mathrm{F}}|f\rangle=-|f\rangle .
$$

Because of the cancellation of contributions of states with positive energies to $\operatorname{Tr}(-1)^{\mathrm{F}}$, this trace formally can be evaluated using the zero-energy states

$$
\operatorname{Tr}(-1)^{\mathrm{F}}=n_{b}^{E=0}-n_{f}^{E=0}
$$

where $n_{b}^{E=0}$ and $n_{f}^{E=0}$ are the numbers of bosonic and fermionic ground states. Therefore, if $\operatorname{Tr}(-1)^{\mathrm{F}} \neq 0$, supersymmetry is not broken. However, because of the presence of infinitely many states, one needs a regulator commuting with $Q$ (to save cancellations). Then the regularized Witten index is defined as

$$
I_{W}=\operatorname{Tr}\left((-1)^{\mathrm{F}} e^{-\beta H}\right),
$$

and formally it does not depend on the parameter $\beta$.

As to $\mathcal{N}=1$ superconformal theories, there are different possibilities to realize relation (3.15), due to the superconformal operators $S^{\alpha}, \bar{S}^{\dot{\alpha}}$. Namely, one picks a generator $\mathcal{Q}$ with its adjoint $\mathcal{Q}^{\dagger}$, such that

$$
\left\{\mathcal{Q}, \mathcal{Q}^{\dagger}\right\}=2 \mathcal{H}
$$

where $\mathcal{H}$ does not coincide with the Hamiltonian. Then one can consider the subspace of the Hilbert space composed of the BPS states $|\psi\rangle$ annihilated by $\mathcal{H}, \mathcal{H}|\psi\rangle=0$, and define the Witten index $I_{W}=\operatorname{Tr}\left((-1)^{\mathrm{F}} e^{-\beta \mathcal{H}}\right)$. However, the space of such states $|\psi\rangle$ is infinite dimensional and one has to introduce other regulators, which leads to a nontrivial generalization of the index itself.

For $S U(2,2 \mid 1)$ group, there are four non-trivial choices for supercharges $\mathcal{Q}, \mathcal{Q}^{\dagger}$, which can be used for constructing the superconformal index:

$$
\begin{array}{rlrl}
\left\{Q_{1}, S^{1}\right\} & =2\left(H+2 J_{3}+\frac{3}{2} R\right) ; & & \left\{Q_{2}, S^{2}\right\}=2\left(H-2 J_{3}+\frac{3}{2} R\right) ; \\
\left\{\bar{Q}_{1},-\bar{S}^{1}\right\} & =2\left(H-2 \bar{J}_{3}-\frac{3}{2} R\right) ; & \left\{\bar{Q}_{2},-\bar{S}^{2}\right\}=2\left(H+2 \bar{J}_{3}-\frac{3}{2} R\right) .
\end{array}
$$

The generators commuting with the corresponding pairs of supercharges are

$$
\begin{array}{cc}
\bar{M}_{\dot{\beta}}^{\dot{\alpha}}, H+\frac{1}{2} R, P_{2 \dot{\alpha}}, K^{\dot{\alpha} 2} ; & \bar{M}_{\dot{\beta}}^{\dot{\alpha}}, H+\frac{1}{2} R, P_{1 \dot{\alpha}}, K^{\dot{\alpha} 1} ; \\
M_{\alpha}{ }^{\beta}, H-\frac{1}{2} R, P_{\alpha 2}, K^{2 \alpha} ; & M_{\alpha}^{\beta}, H-\frac{1}{2} R, P_{\alpha 1}, K^{1 \alpha},
\end{array}
$$

respectively, see (3.14). Let us stick to the choice

$$
\mathcal{Q}=\bar{Q}_{1}, \quad \mathcal{Q}^{\dagger}=-\bar{S}^{1}, \quad \mathcal{H}=H-2 \bar{J}_{3}-\frac{3}{2} R
$$


Composing the matrix [26]

$$
\mathcal{M}_{A}^{B}=\left(\begin{array}{cc}
M_{\alpha}^{\beta}+\frac{1}{2} \delta_{\alpha}^{\beta} \mathcal{R} & \mathcal{P}_{\alpha} \\
\overline{\mathcal{P}}^{\beta} & -\mathcal{R}+\frac{1}{2} \mathcal{H}
\end{array}\right),
$$

where $\mathcal{P}_{\alpha}=\frac{1}{2} P_{\alpha 2}, \overline{\mathcal{P}}^{\beta}=\frac{1}{2} K^{2 \beta}$, and

$$
\mathcal{R}=H-\frac{1}{2} R
$$

we come to the $S U(2,1)$ Lie algebra with the relations

$$
\left[\mathcal{M}_{A}^{B}, \mathcal{M}_{C}^{D}\right]=\delta_{C}^{B} \mathcal{M}_{A}^{D}-\delta_{A}^{D} \mathcal{M}_{C}^{B} .
$$

To regularize the trace over the infinite dimensional space of zero modes of $\mathcal{H}$, we use all operators commuting between themselves and with the distinguished supercharges $\mathcal{Q}$ and $\mathcal{Q}^{\dagger}$. In our case one additional regulator is $t^{\mathcal{R}}$ for some arbitrary complex variable $t$ restricted as $|t|<1$ to ensure damping. Since $M_{\alpha}{ }^{\beta}$ commute with $\bar{Q}_{1}$ and $\bar{S}^{1}$, there is one more regulator $x^{2 J_{3}},|x|<1$, resolving the degeneracy ensured by $M_{\alpha}{ }^{\beta}$. Finally, one defines $[72,73]$

$$
\operatorname{ind}(t, x)=\operatorname{Tr}(-1)^{\mathrm{F}} x^{2 J_{3}} t^{\mathcal{R}} e^{-\beta \mathcal{H}} .
$$

This index explicitly depends on the chemical potentials $x$ and $t$, in difference from the variable $\beta$.

In the presence of internal symmetries, one can introduce more regulators to resolve the degeneracies. For $U(1)^{k}$ global symmetry group, one introduces chemical potentials $\mu_{j}, j=$ $1, \ldots, k$, and extends the superconformal index as

$$
\operatorname{ind}\left(t, x, \mu_{j}\right)=\operatorname{Tr}(-1)^{\mathrm{F}} x^{2 J_{3}} t^{\mathcal{R}} e^{\sum_{j=1}^{k} \mu_{j} q_{j}},
$$

where $q_{j}$ is the generator of $j$-th $U(1)$-group. For a non-abelian local gauge invariance group $G$ with the maximal torus generators $G_{a}, a=1, \ldots$, rank $G$, and a flavor group $F$ with the maximal torus generators $F_{j}, j=1, \ldots$, rank $F$, the index reads

$$
\operatorname{ind}(t, x, z, y)=\operatorname{Tr}\left((-1)^{\mathrm{F}} x^{2 J_{3}} t^{\mathcal{R}} e^{\sum_{a=1}^{r a n k} g_{a} G^{a}} e^{\sum_{j=1}^{r a n k F} f_{j} F^{j}}\right)
$$

where $g_{a}$ and $f_{j}$ are the chemical potentials for groups $G$ and $F$ respectively. We assume that the global abelian groups enter the flavor group contributions in (3.25). From the representation theory it is known that $\operatorname{Tr} \exp \left(\sum_{i=1}^{\operatorname{rank} G} g_{i} G^{i}\right)=\chi_{G}(z)$ is the character of the corresponding representation of the gauge group $G$, where $z$ is the set of complex eigenvalues of matrices realizing $G$. The same is valid for the flavor group $F: \operatorname{Tr} \exp \left(\sum_{j=1}^{\operatorname{rank} M} f_{j} F^{j}\right)=\chi_{F}(y)$ is the character of the representations forming the space of free field states, and $y$ is the set of complex eigenvalues of matrices realizing $F$.

Since all physical observables are gauge invariant, one is interested in the index for gauge singlet operators. Therefore formula (3.25) is averaged over the gauge group, which yields the matrix integral

$$
I(t, x, y)=\int_{G} d \mu(g) \operatorname{Tr}\left((-1)^{\mathrm{F}} x^{2 J_{3}} t^{\mathcal{R}} e^{\sum_{a=1}^{r a n k G} g_{a} G^{a}} e^{\sum_{j=1}^{r a n k F} f_{j} F^{j}}\right),
$$

where $d \mu(g)$ is the $G$-invariant matrix group measure. This is the superconformal index - the key object for our purposes. By construction, it has the meaning of a particular combination of $S U(2,2 \mid 1) \times G \times F$ group characters naturally restricted to the space of BPS states and integrated over the gauge group. 
3.3. Calculation of the index. Explicit computation of the superconformal index for $\mathcal{N}=1$ theories was performed by Römelsberger [73]. According to his prescription one should first compute the trace in index (3.25) over the single particle states, which yields the formula

$$
\begin{aligned}
i(t, x, z, y) & =\frac{2 t^{2}-t\left(x+x^{-1}\right)}{(1-t x)\left(1-t x^{-1}\right)} \chi_{a d j}(z) \\
& +\sum_{j} \frac{t^{2 r_{j}} \chi_{R_{F}, j}(y) \chi_{R_{G}, j}(z)-t^{2-2 r_{j}} \chi_{\bar{R}_{F}, j}(y) \chi_{\bar{R}_{G}, j}(z)}{(1-t x)\left(1-t x^{-1}\right)}
\end{aligned}
$$

where the first term represents contribution of the gauge fields belonging to the adjoint representation of the group $G$, and the sum over $j$ corresponds to the chiral matter superfields $\varphi_{j}$ transforming as the gauge group representations $R_{G, j}$ and non-abelian flavor symmetry group representations $R_{F, j}$. The functions $\chi_{a d j}(z), \chi_{R_{F}, j}(y)$ and $\chi_{R_{G}, j}(z)$ are the corresponding characters - their explicit forms for major classical groups are described in Appendix A.

In the original Romelsberger's formula the denominators are written as $1-t \chi_{S U(2), f}(\gamma)+t^{2}$, where $\chi_{S U(2), f}(\gamma)$ is the character for the fundamental representation of the $S U(2)$ subgroup in (3.22). Parametrizing it by the eigenvalue $x$, one comes to (3.27).

The $U(1)_{R}$-group contribution to (3.27) is described by the terms $t^{2 R_{j}}$ and $t^{2-2 R_{j}}$ resulting from a chiral scalar field with the $R$-charge $2 R_{j}$ and the fermion partner of the conjugate antichiral fields whose $R$-charge is $-2 R_{j}$. In the presence of additional global $U(1)$-groups the variables $r_{j}$ have the form

$$
r_{j}=R_{j}+\sum_{l=1}^{k} q_{j l} \mu_{l}
$$

where $2 R_{j}$ is the $R$-charge of the $j$-th chiral superfield, $q_{j l}$ are the normalized hypercharges of the $j$-th matter superfield for $l$-th $U(1)$-group and $2 \mu_{l}$ is the chemical potential for the latter group.

To obtain the full superconformal index, this single particle states index is inserted into the "plethystic" exponential with the subsequent averaging over the gauge group:

$$
I(t, x, y)=\int_{G} d \mu(g) \exp \left(\sum_{n=1}^{\infty} \frac{1}{n} i\left(t^{n}, x^{n}, z^{n}, y^{n}\right)\right) .
$$

Similar objects appeared in computation of partition functions of different statistical mechanics models and quantum field theories, see, e.g., [3, 80, 95, 58, 49, 57, 4, 29, 24].

Clearly there are two qualitatively different contributions to superconformal indices - from the matter fields and the gauge fields. The generic form of a matter field single particle states contribution to $i(t, x, z, y)$ in the presence of some global $U(1)$ symmetry group is

$$
i_{S}(t, x, y)=\frac{t^{2 R} y-t^{2-2 R} y^{-1}}{(1-t x)\left(1-t x^{-1}\right)},
$$

where $t, x$ are the same variables as in (3.27) and $y=t^{2 \mu}$ is the chemical potential for the $U(1)$ group. It is convenient to introduce new parametrization

$$
p=t x, \quad q=t x^{-1}, \quad w=t^{2 R} y,
$$

where $p$ and $q$ are (in general, complex) parameters satisfying the constraints $|q|,|p|<1$. As a result, we can write

$$
i_{S}(p, q, w)=\frac{w-p q w^{-1}}{(1-p)(1-q)}
$$


Then the described index building algorithm yields (cf. [3])

$$
\exp \left(\sum_{n=1}^{\infty} \frac{1}{n} i_{S}\left(p^{n}, q^{n}, w^{n}\right)\right)=\prod_{j, k=0}^{\infty} \frac{1-w^{-1} p^{j+1} q^{k+1}}{1-w p^{j} q^{k}}=: \Gamma(w ; p, q) .
$$

This result corresponds to formula (69) in [73] after the identifications $w:=t^{q} u, p:=t y, q:=$ $t y^{-1}$. However, the fact that this index coincides with the elliptic gamma function was recognized only by Dolan and Osborn in [26].

For the gauge field part one can set

$$
i_{V}(p, q, z)=\frac{2 t^{2}-t\left(x+x^{-1}\right)}{(1-t x)\left(1-t x^{-1}\right)} \chi_{a d j}(z)=\left(-\frac{p}{1-p}-\frac{q}{1-q}\right) \chi_{a d j}(z) .
$$

For the $S U(2)$ group one has $\chi_{a d j}(z)=z^{2}+z^{-2}+1$. Substituting pieces of this expression in the corresponding places of the index, we obtain the following characteristic building blocks

$$
\begin{gathered}
\exp \left(\sum_{n=1}^{\infty} \frac{1}{n}\left(-\frac{p^{n}}{1-p^{n}}-\frac{q^{n}}{1-q^{n}}\right)\left(z^{2 n}+z^{-2 n}\right)\right)=\frac{\theta\left(z^{2} ; p\right) \theta\left(z^{2} ; q\right)}{\left(1-z^{2}\right)^{2}} \\
=\frac{1}{\left(1-z^{2}\right)\left(1-z^{-2}\right) \Gamma\left(z^{ \pm 2} ; p, q\right)}
\end{gathered}
$$

and

$$
\exp \left(\sum_{n=1}^{\infty} \frac{1}{n}\left(-\frac{p^{n}}{1-p^{n}}-\frac{q^{n}}{1-q^{n}}\right)\right)=(p ; p)_{\infty}(q ; q)_{\infty}
$$

Similar expressions are found for field contributions for the higher rank gauge groups.

\section{Seiberg Duality For unitary Gauge groups}

First we consider the usual $\mathcal{N}=1$ supersymmetric quantum chromodynamics (SQCD) as an electric theory with the internal symmetry groups [76]

$$
G=S U(N), \quad F=S U\left(N_{f}\right) \times S U\left(N_{f}\right) \times U(1)_{B}
$$

where $U(1)_{B}$ is generated by the baryon number charge (the $U(1)_{R}$ group enters the superconformal group). This supersymmetric version of QCD has two chiral scalar multiplets $Q$ and $\tilde{Q}$ belonging to the fundamental $f$ and anti-fundamental $\bar{f}$ representations of $S U(N)$ respectively, each carrying a baryon number, and the vector multiplet $V$ in the adjoint representation of $G$. The field content of the electric theory is collected in the following table

\begin{tabular}{|c|c|c|c|c|c|}
\hline Field & $S U(N)$ & $S U\left(N_{f}\right)$ & $S U\left(N_{f}\right)$ & $U(1)_{B}$ & $U(1)_{R}$ \\
\hline$Q$ & $f$ & $f$ & 1 & $q_{B}=1$ & $2 R_{Q}=\tilde{N} / N_{f}$ \\
$\tilde{Q}$ & $\bar{f}$ & 1 & $\bar{f}$ & $\widetilde{q}_{B}=-1$ & $2 R_{\widetilde{Q}}=\tilde{N} / N_{f}$ \\
$V$ & adj & 1 & 1 & 0 & $2 R_{V}=1$ \\
\hline
\end{tabular}

Here $q_{B}, \widetilde{q}_{B}$ denote the baryonic charge and $R_{Q}, R_{\widetilde{Q}}, R_{V}$ are half $R$-charges of the fields.

The dual magnetic theory has the symmetry groups

$$
G=S U(\tilde{N}), \quad F=S U\left(N_{f}\right) \times S U\left(N_{f}\right) \times U(1)_{B},
$$

where $\tilde{N}=N_{f}-N$. Its field content is fixed in the table below 


\begin{tabular}{|c|c|c|c|c|c|}
\hline Field & $S U(\tilde{N})$ & $S U\left(N_{f}\right)$ & $S U\left(N_{f}\right)$ & $U(1)_{B}$ & $U(1)_{R}$ \\
\hline$q$ & $f$ & $\bar{f}$ & 1 & $q_{B}^{\prime}=N / \tilde{N}$ & $2 R_{q}=N / N_{f}$ \\
$\tilde{q}$ & $\bar{f}$ & 1 & $f$ & $\widetilde{q}_{B}^{\prime}=-N / \tilde{N}$ & $2 R_{\tilde{q}}=N / N_{f}$ \\
$M$ & 1 & $f$ & $\bar{f}$ & 0 & $2 R_{M}=2 \tilde{N} / N_{f}$ \\
$\tilde{V}$ & adj & 1 & 1 & 0 & $2 R_{V}=1$ \\
\hline
\end{tabular}

This duality is supposed to work only in the conformal window $3 N / 2<N_{f}<3 N$, following from the demand that both dual theories are asymptotically free in the one-loop approximation. The one-loop beta function for the gauge coupling is given by

$$
\beta_{g}=-\frac{g^{3}}{16 \pi^{2}}\left(\frac{11}{3} T(\mathbf{a d j})-\frac{2}{3} T(F)-\frac{1}{3} T(S)\right),
$$

where $T(F)$ is the sum of Casimir coefficients $T(\mathbf{r})$ (see Appendix $\mathrm{C}$ for more details) over all fermions, $T(S)$ is the similar sum over all scalars and $T(\mathbf{a d j})$ is $T(\mathbf{r})$ for the adjoint representation. For a summary of this and two loop renormalization group results, see [56].

The $r_{j}$-charges of fields coming from $U(1)_{R}$ and $U(1)_{B}$ currents in the electric theory are

$$
r_{Q}=R_{Q}+q_{B} x, \quad r_{\widetilde{Q}}=R_{\widetilde{Q}}+\widetilde{q}_{B} x,
$$

where $x$ is the $U(1)_{B}$-group chemical potential. In the magnetic theory we set

$$
r_{q}=R_{q}+q_{B}^{\prime} x, \quad r_{\widetilde{q}}=R_{\widetilde{q}}+\widetilde{q}_{B} x, \quad r_{M}=R_{M} .
$$

Then the single particle states index for the electric theory has the form

$$
\begin{aligned}
& i_{E}(p, q, z, s, t)=-\left(\frac{p}{1-p}+\frac{q}{1-q}\right) \chi_{S U(N), a d j}(z) \\
& \quad+\frac{1}{(1-p)(1-q)}\left((p q)^{r_{Q}} \chi_{S U\left(N_{f}\right), f}(s) \chi_{S U(N), f}(z)-(p q)^{1-r_{Q}} \chi_{S U\left(N_{f}\right), \bar{f}}(s) \chi_{S U(N), \bar{f}}(z)\right. \\
& \left.\quad+(p q)^{r} \widetilde{Q} \chi_{S U\left(N_{f}\right), \bar{f}}(t) \chi_{S U(N), \bar{f}}(z)-(p q)^{1-r_{\widetilde{Q}}} \chi_{S U\left(N_{f}\right), f}(t) \chi_{S U(N), f}(z)\right) .
\end{aligned}
$$

For the magnetic theory we have

$$
\begin{aligned}
& i_{M}(p, q, z, s, t)=-\left(\frac{p}{1-p}+\frac{q}{1-q}\right) \chi_{S U(\widetilde{N}), a d j}(z) \\
& \quad+\frac{1}{(1-p)(1-q)}\left((p q)^{r_{q}} \chi_{S U\left(N_{f}\right), \bar{f}}(s) \chi_{S U(\widetilde{N}), f}(z)-(p q)^{1-r_{q}} \chi_{S U\left(N_{f}\right), f}(s) \chi_{S U(\widetilde{N}), \bar{f}}(z)\right. \\
& \quad+(p q)^{r_{\widetilde{q}}} \chi_{S U\left(N_{f}\right), f}(t) \chi_{S U(\widetilde{N}), \bar{f}}(z)-(p q)^{1-r_{\widetilde{q}}} \chi_{S U\left(N_{f}\right), \bar{f}}(t) \chi_{S U(\widetilde{N}), f}(z) \\
& \left.\quad+(p q)^{r_{M}} \chi_{S U\left(N_{f}\right), f}(s) \chi_{S U\left(N_{f}\right), \bar{f}}(t)-(p q)^{1-r_{M}} \chi_{S U\left(N_{f}\right), \bar{f}}(s) \chi_{S U\left(N_{f}\right), f}(t)\right)
\end{aligned}
$$

The superconformal indices take the form (see the invariant measures in Appendix B)

$$
\begin{aligned}
I_{E}= & \frac{(p ; p)_{\infty}^{N-1}(q ; q)_{\infty}^{N-1}}{N !} \\
& \times \int_{\mathbb{T}^{N-1}} \frac{\prod_{i=1}^{N_{f}} \prod_{j=1}^{N} \Gamma\left((p q)^{r_{Q}} s_{i} z_{j},(p q)^{\widetilde{r}_{Q}} t_{i}^{-1} z_{j}^{-1} ; p, q\right)}{\prod_{1 \leq i<j \leq N} \Gamma\left(z_{i} z_{j}^{-1}, z_{i}^{-1} z_{j} ; p, q\right)} \prod_{j=1}^{N-1} \frac{d z_{j}}{2 \pi \mathrm{i} z_{j}},
\end{aligned}
$$


where $\prod_{j=1}^{N} z_{j}=\prod_{i=1}^{N_{f}} s_{i}=\prod_{i=1}^{N_{f}} t_{i}=1$, and

$$
\begin{aligned}
I_{M}= & \frac{(p ; p)_{\infty}^{\widetilde{N}-1}(q ; q)_{\infty}^{\widetilde{N}-1}}{\widetilde{N} !} \prod_{1 \leq i, j \leq N_{f}} \Gamma\left((p q)^{r_{M}} s_{i} t_{j}^{-1} ; p, q\right) \\
& \times \int_{\mathbb{T}^{\tilde{N}-1}} \frac{\prod_{i=1}^{N_{f}} \prod_{j=1}^{\widetilde{N}} \Gamma\left((p q)^{r_{q}} s_{i}^{-1} z_{j},(p q)^{r_{\tilde{q}}} t_{i} z_{j}^{-1} ; p, q\right)}{\prod_{1 \leq i<j \leq \widetilde{N}} \Gamma\left(z_{i} z_{j}^{-1}, z_{i}^{-1} z_{j} ; p, q\right)} \prod_{j=1}^{\widetilde{N}-1} \frac{d z_{j}}{2 \pi \mathrm{i} z_{j}},
\end{aligned}
$$

where $\prod_{j=1}^{\widetilde{N}} z_{j}=1$. Let us renormalize the variables

$$
s_{i} \rightarrow(p q)^{-r_{Q}} s_{i}, \quad t_{i}^{-1} \rightarrow(p q)^{-r} \tilde{Q} t_{i}^{-1}, \quad i=1, \ldots, N_{f} .
$$

Then the superconformal indices are rewritten as the following elliptic hypergeometric integrals

$$
\begin{aligned}
I_{E}= & \frac{(p ; p)_{\infty}^{N-1}(q ; q)_{\infty}^{N-1}}{N !} \\
& \times \int_{\mathbb{T}^{N-1}} \frac{\prod_{i=1}^{N_{f}} \prod_{j=1}^{N} \Gamma\left(s_{i} z_{j}, t_{i}^{-1} z_{j}^{-1} ; p, q\right)}{\prod_{1 \leq i<j \leq N} \Gamma\left(z_{i} z_{j}^{-1}, z_{i}^{-1} z_{j} ; p, q\right)} \prod_{j=1}^{N-1} \frac{d z_{j}}{2 \pi \mathrm{i} z_{j}}
\end{aligned}
$$

and

$$
\begin{aligned}
I_{M}= & \frac{(p ; p)_{\infty}^{\widetilde{N}-1}(q ; q)_{\infty}^{\widetilde{N}-1}}{\widetilde{N} !} \prod_{1 \leq i, j \leq N_{f}} \Gamma\left(s_{i} t_{j}^{-1} ; p, q\right) \\
& \times \int_{\mathbb{T}^{\widetilde{N}-1}} \frac{\prod_{i=1}^{N_{f}} \prod_{j=1}^{\widetilde{N}} \Gamma\left(S^{1 / \widetilde{N}} s_{i}^{-1} z_{j}, T^{-1 / \widetilde{N}} t_{i} z_{j}^{-1} ; p, q\right)}{\prod_{1 \leq i<j \leq \widetilde{N}} \Gamma\left(z_{i} z_{j}^{-1}, z_{i}^{-1} z_{j} ; p, q\right)} \prod_{j=1}^{\widetilde{N}-1} \frac{d z_{j}}{2 \pi \mathrm{i} z_{j}},
\end{aligned}
$$

where $S=\prod_{i=1}^{N_{f}} s_{i}, T=\prod_{i=1}^{N_{f}} t_{i}$, and the balancing condition reads $S T^{-1}=(p q)^{N_{f}-N}$.

As shown by Dolan and Osborn [26], the equality $I_{E}=I_{M}$ coincides with the $A_{n} \leftrightarrow A_{m}$ root systems symmetry transformation established by Rains [65]. For $N=\widetilde{N}=2$ this identity is a simple consequence of the symmetry transformation for an elliptic analogue of the Gauss hypergeometric function discovered earlier by the first author in [83]. Note that this equality of integrals is valid for any $N_{f}$, while the Seiberg duality is expected to exist only in the conformal window, where we have appropriate $R$-charges yielding an anomaly free theory. One cannot extrapolate the duality outside this window except of the boundary points $N_{f}=3 N / 2$ and $N_{f}=3 N$ (we thank A. Schwimmer and S. Theisen for a discussion on this point). However, this does not mean that for the electric theory outside the conformal window there cannot be different magnetic duals. We present a number of such examples in a separate paper [92].

The needed equality between elliptic hypergeometric integrals is rigorous only under certain constraints on the parameters. The kernels of the integrals are meromorphic functions of integration variables $z_{j} \in \mathbb{C}^{*}$. There are two qualitatively different geometric sequences of poles of these kernels - some of them converge to zero $z_{j}=0$ and others go to infinity. So, the equality $I_{E}=I_{M}$ with the integration contours $\mathbb{T}$ on both sides is true provided $\mathbb{T}$ separates these two types of pole sequences. In the present situation this is guaranteed for $|S|^{1 / \tilde{N}}<\left|s_{i}\right|<1$ and $1<\left|t_{i}\right|<|T|^{1 / \tilde{N}}$. All the relations for superconformal indices described below have similar constraints on the parameters, but we shall not describe them for brevity, assuming that the separability conditions for pole sequences are satisfied by the contour $\mathbb{T}$. 


\section{Intriligator-Pouliot DUALity FOR SyMPLECTIC GAUGE Groups}

The electric theory has the overall symmetry group

$$
G=S P(2 N), \quad F=S U\left(2 N_{f}\right),
$$

and the following matter field content

\begin{tabular}{|l|c|c|c|}
\hline & $S P(2 N)$ & $S U\left(2 N_{f}\right)$ & $U(1)_{R}$ \\
\hline$Q$ & $f$ & $f$ & $1-(N+1) / N_{f}$ \\
\hline
\end{tabular}

In this and all other tables below we drop the vector superfields $V$ (or $\widetilde{V}$, except for the confining theories where this field is absent), since they are always described by the adjoint representation of $G$ and singlets of $F$.

The dual magnetic theory constructed by Intriligator and Pouliot [43] has the same flavor group and the gauge group $G=S P(2 \widetilde{N})$, where $\widetilde{N}=N_{f}-N-2$, with the field content described in the table below

\begin{tabular}{|c|c|c|c|}
\hline & $S P(2 \widetilde{N})$ & $S U\left(2 N_{f}\right)$ & $U(1)_{R}$ \\
\hline$q$ & $f$ & $\bar{f}$ & $(N+1) / N_{f}$ \\
$M$ & 1 & $T_{A}$ & $2(\widetilde{N}+1) / N_{f}$ \\
\hline
\end{tabular}

The conformal window for this duality is $3(N+1) / 2<N_{f}<3(N+1)$.

For these theories we have the following indices (in the renormalized variables) [26]

$$
I_{E}=\frac{(p ; p)_{\infty}^{N}(q ; q)_{\infty}^{N}}{2^{N} N !} \int_{\mathbb{T}^{N}} \frac{\prod_{i=1}^{2 N_{f}} \prod_{j=1}^{N} \Gamma\left(t_{i} z_{j}^{ \pm 1} ; p, q\right)}{\prod_{1 \leq i<j \leq N} \Gamma\left(z_{i}^{ \pm 1} z_{j}^{ \pm 1} ; p, q\right) \prod_{j=1}^{N} \Gamma\left(z_{j}^{ \pm 2} ; p, q\right)} \prod_{j=1}^{N} \frac{d z_{j}}{2 \pi \mathrm{i} z_{j}}
$$

and

$$
\begin{aligned}
I_{M}= & \frac{(p ; p)_{\infty}^{\tilde{N}}(q ; q)_{\infty}^{\tilde{N}}}{2^{\tilde{N}} \widetilde{N} !} \prod_{1 \leq i<j \leq 2 N_{f}} \Gamma\left(t_{i} t_{j} ; p, q\right) \\
& \times \int_{\mathbb{N}^{\tilde{N}}} \frac{\prod_{i=1}^{2 N_{f}} \prod_{j=1}^{\widetilde{N}} \Gamma\left((p q)^{1 / 2} t_{i}^{-1} z_{j}^{ \pm 1} ; p, q\right)}{\prod_{1 \leq i<j \leq \widetilde{N}} \Gamma\left(z_{i}^{ \pm 1} z_{j}^{ \pm 1} ; p, q\right) \prod_{j=1}^{\tilde{N}} \Gamma\left(z_{j}^{ \pm 2} ; p, q\right)} \prod_{j=1}^{\widetilde{N}} \frac{d z_{j}}{2 \pi \mathrm{i} z_{j}},
\end{aligned}
$$

with the balancing condition $\prod_{i=1}^{2 N_{f}} t_{i}=(p q)^{N_{f}-N-1}$. For $N=\widetilde{N}=1$, the equality $I_{E}=I_{M}$ is a consequence of the symmetry transformation established in [83]. For arbitrary ranks $N, \widetilde{N}$, the needed identity (2.11) was proven by Rains in [65]. After the degeneration to the rational integrals level, it reduces to the Dixon transformation formula [23].

\section{Multiple Duality for $S P(2 N)$ gauge group}

There exists a multiple duality phenomenon, when one electric theory has many magnetic duals. In this section we describe theories with $S P(2 N)$ gauge group, where multiple duality is ensured by $W\left(E_{7}\right)$, the Weyl group for the exceptional root system $E_{7}$. However, we skip the description of this group referring for details to [91].

We take $\mathcal{N}=1$ SQCD electric theory with the symmetry groups $G=S P(2 N)$ and $F=S U(8) \times U(1)$. This model has one chiral scalar multiplet $Q$ belonging to the fundamental representations of $G$ and $F$, a vector multiplet $V$ in the adjoint representation, and the antisymmetric $S P(2 N)$-tensor field $X$, see the table below 


\begin{tabular}{|c|c|c|c|c|}
\hline & $S P(2 N)$ & $S U(8)$ & $U(1)$ & $U(1)_{R}$ \\
\hline$Q$ & $f$ & $f$ & $-\frac{N-1}{4}$ & $\frac{1}{2}$ \\
$X$ & $T_{A}$ & 1 & 1 & 0 \\
\hline
\end{tabular}

For $N=1$, the field $X$ is absent and $U(1)$-group is completely decoupled. In [91] we were giving in tables halves of the $R$-charges.

This electric theory and its particular magnetic dual (with $N>1$ ) were considered in [18]. However, as described in [91], there are other dual theories. In a special section below we show that the 't Hooft anomaly matching conditions are fulfilled for all these new dualities.

The electric superconformal index is

$$
\begin{gathered}
I_{E}=\frac{(p ; p)_{\infty}^{N}(q ; q)_{\infty}^{N} \Gamma\left((p q)^{s} ; p, q\right)^{N-1} \int_{\mathbb{T}^{N}} \prod_{1 \leq i<j \leq N} \frac{\Gamma\left((p q)^{s} z_{i}^{ \pm 1} z_{j}^{ \pm 1} ; p, q\right)}{\Gamma\left(z_{i}^{ \pm 1} z_{j}^{ \pm 1} ; p, q\right)}}{2^{N} N !} \\
\times \prod_{j=1}^{N} \frac{\prod_{i=1}^{8} \Gamma\left((p q)^{r_{Q}} y_{i} z_{j}^{ \pm 1} ; p, q\right)}{\Gamma\left(z_{j}^{ \pm 2} ; p, q\right)} \frac{d z_{j}}{2 \pi \mathrm{i} z_{j}},
\end{gathered}
$$

where $r_{Q}=R_{Q}+e_{Q} s, r_{X}=e_{X} s$, and $2 R_{Q}=1 / 2$ is the $R$-charge of the $Q$-field, $e_{Q}=-(N-1) / 4$ and $e_{X}=1$ are the $U(1)$-group hypercharges with $s$ being its chemical potential.

The first (new) class of magnetic theories has the symmetry groups

$$
G=S P(2 N), \quad F=S U(4) \times S U(4) \times U(1)_{B} \times U(1) .
$$

Its field content is fixed in the table below

\begin{tabular}{|c|c|c|c|c|c|c|}
\hline & $S P(2 N)$ & $S U(4)$ & $S U(4)$ & $U(1)_{B}$ & $U(1)$ & $U(1)_{R}$ \\
\hline$q$ & $f$ & $f$ & 1 & -1 & $-\frac{N-1}{4}$ & $\frac{1}{2}$ \\
$\widetilde{q}$ & $f$ & 1 & $f$ & 1 & $-\frac{N-1}{4}$ & $\frac{1}{2}$ \\
$Y$ & $T_{A}$ & 1 & 1 & 0 & 1 & 0 \\
$M_{J}$ & 1 & $T_{A}$ & 1 & 2 & $\frac{2 J-N+1}{2}$ & 1 \\
$\widetilde{M}_{J}$ & 1 & 1 & $T_{A}$ & -2 & $\frac{2 J-N+1}{2}$ & 1 \\
\hline
\end{tabular}

In the tables of this section the capital index $J$ takes values $0, \ldots, N-1$, which is not indicated for brevity. The superconformal index in this magnetic theory is

$$
\begin{aligned}
I_{M}^{(1)} & =\prod_{J=0}^{N-1} \prod_{1 \leq i<j \leq 4} \Gamma\left((p q)^{r_{M_{J}}} y_{i} y_{j} ; p, q\right) \prod_{5 \leq i<j \leq 8} \Gamma\left((p q)^{r_{\widetilde{M}_{J}}} y_{i} y_{j} ; p, q\right) \\
& \times \Gamma\left((p q)^{s} ; p, q\right)^{N-1} \frac{(p ; p)_{\infty}^{N}(q ; q)_{\infty}^{N}}{2^{N} N !} \int_{\mathbb{T}^{N}} \prod_{1 \leq i<j \leq N} \frac{\Gamma\left((p q)^{s} z_{i}^{ \pm 1} z_{j}^{ \pm 1} ; p, q\right)}{\Gamma\left(z_{i}^{ \pm 1} z_{j}^{ \pm 1} ; p, q\right)} \\
& \times \prod_{j=1}^{N} \frac{\prod_{i=1}^{4} \Gamma\left((p q)^{r_{q}} v^{-2} y_{i} z_{j}^{ \pm 1} ; p, q\right) \prod_{i=5}^{8} \Gamma\left((p q)^{r} \widetilde{q} v^{2} y_{i} z_{j}^{ \pm 1} ; p, q\right)}{\Gamma\left(z_{j}^{ \pm 2} ; p, q\right)} \frac{d z_{j}}{2 \pi \mathrm{i} z_{j}}
\end{aligned}
$$

where $v=\sqrt[4]{y_{1} y_{2} y_{3} y_{4}}$ and

$$
\begin{array}{r}
r_{q}=R_{q}-\frac{N-1}{4} s, \quad r_{\widetilde{q}}=R_{\widetilde{q}}-\frac{N-1}{4} s, \quad r_{Y}=s, \\
r_{M_{J}}=R_{M_{J}}-\frac{1}{2}(N-1-2 J) s, \quad r_{\widetilde{M}_{J}}=R_{\widetilde{M}_{J}}-\frac{1}{2}(N-1-2 J) s .
\end{array}
$$

The second (new) class of dual magnetic theories has the same symmetries as in the previous case, but different representation content as described in the following table 


\begin{tabular}{|c|c|c|c|c|c|c|}
\hline & $S P(2 N)$ & $S U(4)$ & $S U(4)$ & $U(1)_{B}$ & $U(1)$ & $U(1)_{R}$ \\
\hline$q$ & $f$ & $\bar{f}$ & $\frac{1}{f}$ & 1 & $-\frac{N-1}{4}$ & $\frac{1}{2}$ \\
$\widetilde{q}$ & $f$ & 1 & -1 & $-\frac{N-1}{4}$ & $\frac{1}{2}$ \\
$Y$ & $T_{A}$ & 1 & 1 & 0 & 1 & 0 \\
$M_{J}$ & 1 & $f$ & $f$ & 0 & $\frac{2 J-N+1}{2}$ & 1 \\
\hline
\end{tabular}

The index for this magnetic theory is given by

$$
\begin{aligned}
I_{M}^{(2)}= & \Gamma\left((p q)^{s} ; p, q\right)^{N-1} \prod_{J=0}^{N-1} \prod_{i=1}^{4} \prod_{j=5}^{8} \Gamma\left((p q)^{r_{M_{J}}} y_{i} y_{j} ; p, q\right) \\
& \times \frac{(p ; p)_{\infty}^{N}(q ; q)_{\infty}^{N}}{2^{N} N !} \int_{\mathbb{T}^{N}} \prod_{1 \leq i<j \leq N} \frac{\Gamma\left((p q)^{s} z_{i}^{ \pm 1} z_{j}^{ \pm 1} ; p, q\right)}{\Gamma\left(z_{i}^{ \pm 1} z_{j}^{ \pm 1} ; p, q\right)} \\
& \times \prod_{j=1}^{N} \frac{\prod_{i=1}^{4} \Gamma\left((p q)^{r_{q}} v^{2} y_{i}^{-1} z_{j}^{ \pm 1} ; p, q\right) \prod_{i=5}^{8} \Gamma\left((p q)^{r_{\widetilde{q}}} v^{-2} y_{i}^{-1} z_{j}^{ \pm 1} ; p, q\right)}{\Gamma\left(z_{j}^{ \pm 2} ; p, q\right)} \frac{d z_{j}}{2 \pi \mathrm{i} z_{j}}
\end{aligned}
$$

where

$$
r_{q}=r_{\widetilde{q}}=\frac{1}{4}-\frac{N-1}{4} s, \quad r_{Y}=s, \quad r_{M_{J}}=\frac{1}{2}-\frac{1}{2}(N-1-2 J) s .
$$

Finally, the third type of magnetic theories, which was constructed originally by Csáki, Skiba and Schmaltz in [18], has the symmetry groups $G=S P(2 N)$ and $F=S U(8) \times U(1)$, and its fields content is

\begin{tabular}{|c|c|c|c|c|}
\hline & $S P(2 N)$ & $S U(8)$ & $U(1)$ & $U(1)_{R}$ \\
\hline$q$ & $f$ & $\bar{f}$ & $-\frac{N-1}{4}$ & $\frac{1}{2}$ \\
$Y$ & $T_{A}$ & 1 & 1 & 0 \\
$M_{J}$ & 1 & $T_{A}$ & $\frac{2 J-N+1}{2}$ & 1 \\
\hline
\end{tabular}

Corresponding magnetic superconformal index has the form

$$
\begin{aligned}
I_{M}^{(3)}= & \Gamma\left((p q)^{r_{Y}} ; p, q\right)^{N-1} \prod_{J=0}^{N-1} \prod_{1 \leq i<j \leq 8} \Gamma\left((p q)^{r_{M_{J}}} y_{i} y_{j} ; p, q\right) \frac{(p ; p)_{\infty}^{N}(q ; q)_{\infty}^{N}}{2^{N} N !} \\
& \times \int_{\mathbb{T}^{N}} \prod_{1 \leq i<j \leq N} \frac{\Gamma\left((p q)^{r_{Y}} z_{i}^{ \pm 1} z_{j}^{ \pm 1} ; p, q\right)}{\Gamma\left(z_{i}^{ \pm 1} z_{j}^{ \pm 1} ; p, q\right)} \prod_{j=1}^{N} \frac{\prod_{i=1}^{8} \Gamma\left((p q)^{r_{q}} y_{i}^{-1} z_{j}^{ \pm 1} ; p, q\right)}{\Gamma\left(z_{j}^{ \pm 2} ; p, q\right)} \frac{d z_{j}}{2 \pi \mathrm{i} z_{j}}
\end{aligned}
$$

where

$$
r_{q}=\frac{1-s(N-1)}{4}, \quad r_{Y}=s, \quad r_{M_{J}}=s J+\frac{1-s(N-1)}{2} .
$$

The $S P(2)$ gauge group case can be obtained from the tables above by substituting $N=1$ and deleting fields $X$ in the electric theory and $Y$ in the magnetic theories, which decouple completely. The number of mesons in dual theories is reduced as well. Equality of superconformal indices for $N=1$ follows from the results of [83], and the needed identities for elliptic hypergeometric integrals for $N>1$ were established in [65]. As argued in [91], there should be in total 72 theories dual to each other - this number equals to the dimension of the coset group $W\left(E_{7}\right) / S_{8}$ responsible for the dualities (in this respect, see also [55]).

\section{A NEW $S P(2 N) \leftrightarrow S P(2 M)$ GROUPS DUALITY}

We take as the electric theory SQCD based on the symmetry groups

$$
G=S P(2 M), \quad F=S U(4) \times S P\left(2 l_{1}\right) \times S P\left(2 l_{2}\right) \times \ldots \times S P\left(2 l_{K}\right) \times U(1)
$$


with the fields content fixed in the table below

\begin{tabular}{|c|c|c|c|c|c|c|c|c|}
\hline & $S P(2 M)$ & $S U(4)$ & $S P\left(2 l_{1}\right)$ & $S P\left(2 l_{2}\right)$ & $\ldots$ & $S P\left(2 l_{K}\right)$ & $U(1)$ & $U(1)_{R}$ \\
\hline$W_{1}$ & $f$ & $\bar{f}$ & 1 & 1 & $\ldots$ & 1 & $-\frac{M-N-2}{4}$ & 0 \\
$Q_{1}$ & $f$ & 1 & $f$ & 1 & $\ldots$ & 1 & $-\frac{n_{1}}{2}$ & 1 \\
$Q_{1}$ & $f$ & 1 & 1 & $f$ & $\ldots$ & 1 & $-\frac{n_{2}}{2}$ & 1 \\
$\ldots$ & & & & & & & & \\
$Q_{K}$ & $f$ & 1 & 1 & 1 & $\ldots$ & $f$ & $-\frac{n_{K}}{2}$ & 1 \\
$X$ & $T_{A}$ & 1 & 1 & 1 & $\ldots$ & 1 & 1 & 0 \\
\hline
\end{tabular}

where $n_{1} \neq n_{2} \neq \ldots \neq n_{K}$ and $\sum_{i=1}^{K} l_{i} n_{i}=M+N$.

The dual magnetic theory has $G=S P(2 N)$ and the same flavor group; the fields content is described below

\begin{tabular}{|c|c|c|c|c|c|c|c|c|}
\hline & $S P(2 N)$ & $S U(4)$ & $S P\left(2 l_{1}\right)$ & $S P\left(2 l_{2}\right)$ & $\ldots$ & $S P\left(2 l_{K}\right)$ & $U(1)$ & $U(1)_{R}$ \\
\hline$w_{1}$ & $f$ & $f$ & 1 & 1 & $\ldots$ & 1 & $\frac{M-N+2}{4}$ & 0 \\
\hline$q_{1}$ & $f$ & 1 & $f$ & 1 & $\ldots$ & 1 & $-\frac{n_{1}}{2}$ & 1 \\
\hline$q_{1}$ & $f$ & 1 & 1 & $f$ & .. & 1 & $-\frac{n_{2}}{2}$ & 1 \\
\hline$q_{K}$ & $f$ & 1 & 1 & 1 & $\ldots$ & $f$ & $-\frac{n_{K}}{2}$ & 1 \\
\hline$N_{j}$ & 1 & $\bar{T}_{A}$ & 1 & 1 & $\ldots$ & 1 & $j-\frac{M-N-2}{2}$ & 0 \\
\hline$M_{1, k_{1}}$ & 1 & $\bar{f}$ & $f$ & 1 & $\ldots$ & 1 & $-\frac{M-N-2}{4}-\frac{n_{1}}{2}+k_{1}$ & 1 \\
\hline$M_{2, k_{2}}$ & 1 & $\bar{f}$ & 1 & $f$ & $\ldots$ & 1 & $-\frac{M-N-2}{4}-\frac{n_{2}}{2}+k_{2}$ & 1 \\
\hline$M_{K, k_{K}}$ & 1 & $\bar{f}$ & 1 & 1 & $\ldots$ & $f$ & $-\frac{M-N-2}{4}-\frac{n_{K}}{2}+k_{K}$ & 1 \\
\hline$Y$ & $T_{A}$ & 1 & 1 & 1 & $\ldots$ & 1 & $\begin{array}{lll}4 & 1^{2}\end{array}$ & 0 \\
\hline
\end{tabular}

where $j=0, \ldots, M-N-1$ and $k_{i}=0, \ldots, n_{i}-1$ for any $i=1, \ldots, K$. Here we assume that $M \geq N$ (for $M=N$ the fields $N_{j}$ are are absent).

The superconformal indices have the form

$$
\begin{aligned}
I_{E}= & \frac{(p ; p)_{\infty}^{M}(q ; q)_{\infty}^{M}}{2^{M} M !} \Gamma(t ; p, q)^{M-1} \int_{\mathbb{T}^{M}} \prod_{1 \leq i<j \leq M} \frac{\Gamma\left(t z_{i}^{ \pm 1} z_{j}^{ \pm 1} ; p, q\right)}{\Gamma\left(z_{i}^{ \pm 1} z_{j}^{ \pm 1} ; p, q\right)} \\
& \times \prod_{j=1}^{M} \frac{\prod_{k=1}^{4} \Gamma\left(t t_{k}^{-1} z_{j}^{ \pm 1} ; p, q\right) \prod_{r=1}^{K} \prod_{j=1}^{l_{r}} \Gamma\left(s_{r, j} z_{j}^{ \pm 1} ; p, q\right)}{\Gamma\left(z_{j}^{ \pm 2} ; p, q\right) \prod_{r=1}^{K} \prod_{j=1}^{l_{r}} \Gamma\left(t^{n_{r}} s_{r, j} z_{j}^{ \pm 1} ; p, q\right)} \frac{d z_{j}}{2 \pi \mathrm{i} z_{j}}
\end{aligned}
$$

and

$$
\begin{aligned}
I_{M}= & \frac{(p ; p)_{\infty}^{N}(q ; q)_{\infty}^{N}}{2^{N} N !} \Gamma(t ; p, q)^{N-1} \prod_{i=0}^{M-N-1} \prod_{1 \leq k<r \leq 4} \Gamma\left(t^{i+2} t_{k}^{-1} t_{r}^{-1} ; p, q\right) \\
& \times \prod_{r=1}^{4} \prod_{m=1}^{K} \prod_{i=1}^{l_{m}} \prod_{k_{m}=0}^{n_{m}-1} \frac{\Gamma\left(t^{k_{m}+1} t_{r}^{-1} s_{m, i} ; p, q\right)}{\Gamma\left(t^{k_{m}} t_{r} s_{m, i} ; p, q\right)} \int_{\mathbb{T}^{N}} \prod_{1 \leq i<j \leq N} \frac{\Gamma\left(t z_{i}^{ \pm 1} z_{j}^{ \pm 1} ; p, q\right)}{\Gamma\left(z_{i}^{ \pm 1} z_{j}^{ \pm 1} ; p, q\right)} \\
& \times \prod_{j=1}^{N} \frac{\prod_{k=1}^{4} \Gamma\left(t_{k} z_{j}^{ \pm 1} ; p, q\right) \prod_{r=1}^{K} \prod_{j=1}^{l_{r}} \Gamma\left(s_{r, j} z_{j}^{ \pm 1} ; p, q\right)}{\Gamma\left(z_{j}^{ \pm 2} ; p, q\right) \prod_{r=1}^{K} \prod_{j=1}^{l_{r}} \Gamma\left(t^{n_{r}} s_{r, j} z_{j}^{ \pm 1} ; p, q\right)} \frac{d z_{j}}{2 \pi \mathrm{i} z_{j}},
\end{aligned}
$$

with the balancing condition $\prod_{r=1}^{4} t_{r}=t^{2+M-N}$.

We have checked that the anomalies of these two theories match (see below), which is a very strong indication that the theories are dual to each other. This is another new duality that we have found. It has rather complicated structure with the flavor group composed of 
an arbitrary number of simple group components. The renormalization group analysis shows that the asymptotic freedom is present on the electric side for $M>\sum_{i=1}^{K} l_{i} / 2-1$ and on the magnetic side for $N>\sum_{i=1}^{K} l_{i} / 2-1$.

The equality of elliptic hypergeometric integrals $I_{E}=I_{M}$, which gives another argument supporting this duality, coincides with the Rains Conjecture 1 from [68] (it was used as a starting point for the derivation of the described duality). As we have known after the completion of this work, this conjecture is proven recently by van de Bult [9].

\section{Multiple Duality for $S U(2 N)$ gauge group}

We describe now the multiple duality phenomenon for $S U(2 N)$ gauge group. The overall flavor symmetry group of the theories is rather unusual. For $N=1$, this multiple duality coincides with that for $S P(2)$ group, see [91]. For $N>2$, one has $F=S U(4) \times S U(4) \times$ $U(1)_{1} \times U(1)_{2} \times U(1)_{B}$. For $N=2$, the flavor subgroup $U(1)_{1}$ is replaced by $S U(2)$. The field content of the electric theory for $N>2$ is shown in the table below

\begin{tabular}{|c|c|c|c|c|c|c|c|}
\hline & $S U(2 N)$ & $S U(4)$ & $S U(4)$ & $U(1)_{1}$ & $U(1)_{2}$ & $U(1)_{B}$ & $U(1)_{R}$ \\
\hline$Q$ & $f$ & $f$ & 1 & 0 & $2 N-2$ & 1 & $\frac{1}{2}$ \\
$\widetilde{Q}$ & $\bar{f}$ & 1 & $f$ & 0 & $2 N-2$ & -1 & $\frac{1}{2}$ \\
$A$ & $T_{A}$ & 1 & 1 & 1 & -4 & 0 & 0 \\
$\bar{A}$ & $\bar{T}_{A}$ & 1 & 1 & -1 & -4 & 0 & 0 \\
\hline
\end{tabular}

Corresponding superconformal index has the form

$$
\begin{aligned}
I_{E}= & \frac{(p ; p)_{\infty}^{2 N-1}(q ; q)_{\infty}^{2 N-1}}{(2 N) !} \int_{\mathbb{T}^{2 N-1}} \prod_{1 \leq j<k \leq 2 N} \frac{\Gamma\left(U z_{j} z_{k}, V z_{j}^{-1} z_{k}^{-1} ; p, q\right)}{\Gamma\left(z_{j}^{-1} z_{k}, z_{j} z_{k}^{-1} ; p, q\right)} \\
& \times \prod_{j=1}^{2 N} \prod_{k=1}^{4} \Gamma\left(s_{k} z_{j}, t_{k} z_{j}^{-1} ; p, q\right) \prod_{j=1}^{2 N-1} \frac{d z_{j}}{2 \pi \mathrm{i} z_{j}},
\end{aligned}
$$

where $\prod_{j=1}^{2 N} z_{j}=1$ and the balancing condition reads $(U V)^{2 N-2} S T=(p q)^{2}$ with $S=\prod_{k=1}^{4} s_{k}$ and $T=\prod_{k=1}^{4} t_{k}$. This is the two-parameter (higher order) extension of the type II elliptic beta integral for the root system $A_{2 N-1}$ introduced by Spiridonov in [83].

For $N \geq 2$, magnetic dual theories have the same gauge and global symmetry groups. The first (new) dual theory has the field content described for $N>2$ in the table below

\begin{tabular}{|c|c|c|c|c|c|c|c|}
\hline & $S U(2 N)$ & $S U(4)$ & $S U(4)$ & $U(1)_{1}$ & $U(1)_{2}$ & $U(1)_{B}$ & $U(1)_{R}$ \\
\hline$q$ & $f$ & $f$ & 1 & 0 & $2 N-2$ & -1 & $\frac{1}{2}$ \\
$\bar{q}$ & $\bar{f}$ & 1 & $f$ & 0 & $2 N-2$ & 1 & $\frac{1}{2}$ \\
$a$ & $T_{A}$ & 1 & 1 & 1 & -4 & 0 & 0 \\
$\bar{a}$ & $\bar{T}_{A}$ & 1 & 1 & -1 & -4 & 0 & 0 \\
$H_{m}$ & 1 & $T_{A}$ & 1 & -1 & $4 N-8-8 m$ & 2 & 1 \\
$G$ & 1 & $T_{A}$ & 1 & $N-1$ & 0 & 2 & 1 \\
$\bar{H}_{m}$ & 1 & 1 & $T_{A}$ & 1 & $4 N-8-8 m$ & -2 & 1 \\
$\bar{G}$ & 1 & 1 & $T_{A}$ & $-N+1$ & 0 & -2 & 1 \\
\hline
\end{tabular}


where $m=0, \ldots, N-2$. This leads to the magnetic index

$$
\begin{aligned}
I_{M}^{(1)}= & \prod_{1 \leq i<j \leq 4}\left[\Gamma\left(U^{N-1} s_{i} s_{j}, V^{N-1} t_{i} t_{j} ; p, q\right) \prod_{m=0}^{N-2} \Gamma\left(V(U V)^{m} s_{i} s_{j}, U(U V)^{m} t_{i} t_{j} ; p, q\right)\right] \\
& \times \frac{(p ; p)_{\infty}^{2 N-1}(q ; q)_{\infty}^{2 N-1}}{(2 N) !} \int_{\mathbb{T}^{2 N-1}} \prod_{1 \leq j<k \leq 2 N} \frac{\Gamma\left(V z_{j} z_{k}, U z_{j}^{-1} z_{k}^{-1} ; p, q\right)}{\Gamma\left(z_{j}^{-1} z_{k}, z_{j} z_{k}^{-1} ; p, q\right)} \\
& \times \prod_{j=1}^{2 N} \prod_{k=1}^{4} \Gamma\left(\sqrt[4]{T / S} s_{k} z_{j}, \sqrt[4]{S / T} t_{k} z_{j}^{-1} ; p, q\right) \prod_{j=1}^{2 N-1} \frac{d z_{j}}{2 \pi \mathrm{i} z_{j}} .
\end{aligned}
$$

Our second dual theory was found by Csáki et al in [17]. Its field content for $N>2$ is described in the table below

\begin{tabular}{|c|c|c|c|c|c|c|c|}
\hline & $S U(2 N)$ & $S U(4)$ & $S U(4)$ & $U(1)_{1}$ & $U(1)_{2}$ & $U(1)_{B}$ & $U(1)_{R}$ \\
\hline$q$ & $f$ & $\bar{f}$ & 1 & 0 & $2 N-2$ & 1 & $\frac{1}{2}$ \\
$\bar{q}$ & $\bar{f}$ & 1 & $\bar{f}$ & 0 & $2 N-2$ & -1 & $\frac{1}{2}$ \\
$a$ & $T_{A}$ & 1 & 1 & 1 & -4 & 0 & 0 \\
$\bar{a}$ & $\bar{T}_{A}$ & 1 & 1 & -1 & -4 & 0 & 0 \\
$M_{k}$ & 1 & $f$ & $f$ & 0 & $4 N-4-8 k$ & 0 & 1 \\
\hline
\end{tabular}

where $k=0, \ldots, N-1$. Its superconformal index has the form

$$
\begin{aligned}
& I_{M}^{(2)}=\frac{(p ; p)_{\infty}^{2 N-1}(q ; q)_{\infty}^{2 N-1}}{(2 N) !} \prod_{m=0}^{N-1} \prod_{k, l=1}^{4} \Gamma\left((U V)^{m} s_{k} t_{l} ; p, q\right) \\
& \times \int_{\mathbb{T}^{2 N-1}} \prod_{1 \leq j<k \leq 2 N} \frac{\Gamma\left(U z_{j} z_{k}, V z_{j}^{-1} z_{k}^{-1} ; p, q\right)}{\Gamma\left(z_{j}^{-1} z_{k}, z_{j} z_{k}^{-1} ; p, q\right)} \prod_{j=1}^{2 N} \prod_{k=1}^{4} \Gamma\left(\sqrt{S} s_{k}^{-1} z_{j}, \sqrt{T} t_{k}^{-1} z_{j}^{-1} ; p, q\right) \prod_{j=1}^{2 N-1} \frac{d z_{j}}{2 \pi \mathrm{i} z_{j}} .
\end{aligned}
$$

Our third, again new, duality corresponds to the theory described below for $N>2$

\begin{tabular}{|c|c|c|c|c|c|c|c|}
\hline & $S U(2 N)$ & $S U(4)$ & $S U(4)$ & $U(1)_{1}$ & $U(1)_{2}$ & $U(1)_{B}$ & $U(1)_{R}$ \\
\hline$q$ & $f$ & $\bar{f}$ & 1 & 0 & $2 N-2$ & -1 & $\frac{1}{2}$ \\
$\bar{q}$ & $\bar{f}$ & 1 & $\bar{f}$ & 0 & $2 N-2$ & 1 & $\frac{1}{2}$ \\
$a$ & $T_{A}$ & 1 & 1 & 1 & -4 & 0 & 0 \\
$\bar{a}$ & $\bar{T}_{A}$ & 1 & 1 & -1 & -4 & 0 & 0 \\
$M_{k}$ & 1 & $f$ & $f$ & 0 & $4 N-4-8 k$ & 0 & 1 \\
$H_{m}$ & 1 & $T_{A}$ & 1 & -1 & $4 N-8-8 m$ & 2 & 1 \\
$G$ & 1 & $T_{A}$ & 1 & $N-1$ & 0 & 2 & 1 \\
$\bar{H}_{m}$ & 1 & 1 & $T_{A}$ & 1 & $4 N-8-8 m$ & -2 & 1 \\
$\bar{G}$ & 1 & 1 & $T_{A}$ & $-N+1$ & 0 & -2 & 1 \\
\hline
\end{tabular}

where $k=0, \ldots, N-1, m=0, \ldots, N-2$. Its superconformal index reads

$$
\begin{aligned}
& I_{M}^{(3)}=\frac{(p ; p)_{\infty}^{2 N-1}(q ; q)_{\infty}^{2 N-1}}{(2 N) !} \prod_{m=0}^{N-1} \prod_{k, l=1}^{4} \Gamma\left((U V)^{m} s_{k} t_{l} ; p, q\right) \\
& \quad \times \prod_{1 \leq i<j \leq 4}\left[\Gamma\left(U^{N-1} s_{i} s_{j}, V^{N-1} t_{i} t_{j} ; p, q\right) \prod_{m=0}^{N-2} \Gamma\left(V(U V)^{m} s_{i} s_{j}, U(U V)^{m} t_{i} t_{j} ; p, q\right)\right] \\
& \times \int_{\mathbb{T}^{2 N-1}} \prod_{1 \leq j<k \leq 2 N} \frac{\Gamma\left(V z_{j} z_{k}, U z_{j}^{-1} z_{k}^{-1} ; p, q\right)}{\Gamma\left(z_{j}^{-1} z_{k}, z_{j} z_{k}^{-1} ; p, q\right)} \prod_{j=1}^{2 N} \prod_{k=1}^{4} \Gamma\left(\sqrt[4]{S T} s_{k}^{-1} z_{j}, \sqrt[4]{S T} t_{k}^{-1} z_{j}^{-1} ; p, q\right) \prod_{j=1}^{2 N-1} \frac{d z_{j}}{2 \pi \mathrm{i} z_{j}} .
\end{aligned}
$$


From the duality arguments for these field theories, we conjecture that $I_{E}=I_{M}^{(1)}=I_{M}^{(2)}=I_{M}^{(3)}$ under certain constraints on the integral parameters, which yield new powerful elliptic hypergeometric integral identities. Instead of the $W\left(E_{7}\right)$ Weyl group symmetry in parameters, existing for $N=1$, only its subgroup of reflection transformations consistent with the permutational $S_{4} \times S_{4}$ symmetry group survives. Nevertheless, preliminary considerations indicate that these relations should be provable by an appropriate analog of the method used in [65] for proving $W\left(E_{7}\right)$-identities for $B C_{N}$-integrals of type II. We have checked that the reduction from $N_{f}=4$ to $N_{f}=3$ realized by the constraint $s_{4} t_{4}=p q$ reduces superconformal indices to Spiridonov's $A_{2 N-1}$-elliptic beta integral [83], i.e. equality of indices in this case is proven rigorously.

For $N=2$, superconformal indices are given by the same integrals. However, in this case $\prod_{1 \leq j<k \leq 4} f\left(z_{i} z_{j}\right)=\prod_{1 \leq j<k \leq 4} f\left(z_{i}^{-1} z_{j}^{-1}\right)$ for arbitrary function $f(x)$, and the parameters $U$ and $V$ unify to a doublet, meaning that the fields $A$ and $\bar{A}, a$ and $\bar{a}$ unify to fundamentals of the $S U(2)$ group, which replaces the $U(1)_{1}$ flavor subgroup.

An interesting situation occurs in the limit $V \rightarrow 1$ (or $U \rightarrow 1$ ). Some of the poles coming from the integrand factor $\Gamma\left(V z_{i}^{-1} z_{j}^{-1} ; p, q\right)$ approach the integration contour and it is necessary to deform $\mathbb{T}$ before taking this limit. A careful residue calculus shows that in this limit the leading asymptotic contribution to all four superconformal indices are given by the residues of the poles at $z_{j} z_{k}=V \rightarrow 1, j \neq k$. As a result, $N-1$ integrations are taken away, there remain only $N$-dimensional integrals over, say, $z_{2 j-1}, j=1, \ldots, N$, variables. The latter integrals coincide exactly with the indices of four theories appearing in multiple $S P(2 N)$-duality described above. Thus we have shown, that our multiple $S U(2 N)$-dualities contain $S P(2 N)$ dualities as special subcases. The first mathematical observation that the type II hypergeometric identities for $B C_{N}$-root system can be obtained from type II relations for both $A_{2 N-1}$ and $A_{2 N}$ root systems has been done in [94], where various new multiple ${ }_{6} \psi_{6}$ summation formulas on root systems have been suggested. Here we extend this observation to the (expected) relations between type II elliptic hypergeometric integrals. On the physical ground, such a relation between the particular $S U(4)$ and $S P(4)$ gauge group dualities was observed in [17]. Note that the further limit $U=1$ leads to the $S U(2)^{N}$-gauge group theories whose indices are given by $N$-th power of the indices of $S P(2)$-group models with $N_{f}=4$ constructed in [91].

The attempts in [17] to construct an analogous duality for even rank gauge groups $S U(2 N+1)$ have failed. We have succeeded in solving this problem; corresponding results together with the residue calculus details will be presented in a separate paper.

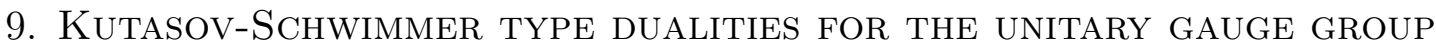

Now we pass to generalizations of the Seiberg dualities for unitary and symplectic gauge groups $G$ discovered by Kutasov and Schwimmer (KS) [51, 52] and studied in detail in [53] and other papers. For brevity, we skip separate global symmetry group descriptions since they can be read off easily from the field contents of the theories given in the tables. The first column in the tables describes gauge group representations for fields, while other columns, except of the very last one, describe representations and hypercharges for subgroups of the flavor group $F$. Also, we skip the detailed description of single particle state indices and write out directly the integrals for the superconformal indices together with the balancing condition, if there is any. In this section we describe such dualities for $G=S U(N)$. 
9.1. $S U(N)$ gauge group with the adjoint matter field. The following electric-magnetic duality is described in [52]. The field content of the electric theory is

\begin{tabular}{|c|c|c|c|c|c|}
\hline & $S U(N)$ & $S U\left(N_{f}\right)$ & $S U\left(N_{f}\right)$ & $U(1)_{B}$ & $U(1)_{R}$ \\
\hline$Q$ & $f$ & $f$ & 1 & 1 & $2 r=1-\frac{2 N}{(K+1) N_{f}}$ \\
$\widetilde{Q}$ & $\bar{f}$ & 1 & $\bar{f}$ & -1 & $2 \widetilde{r}=1-\frac{2 N}{(K+1) N_{f}}$ \\
$X$ & $a d j$ & 1 & 1 & 0 & $2 s=\frac{2}{K+1}$ \\
\hline
\end{tabular}

The magnetic theory ingredients are collected in the following table

\begin{tabular}{|c|c|c|c|c|c|}
\hline & $S U(\widetilde{N})$ & $S U\left(N_{f}\right)$ & $S U\left(N_{f}\right)$ & $U(1)_{B}$ & $U(1)_{R}$ \\
\hline$q$ & $f$ & $\bar{f}$ & 1 & $N / \widetilde{N}$ & $2 r^{\prime}=1-\frac{2 \widetilde{N}}{(K+1) N_{f}}$ \\
$\widetilde{q}$ & $\bar{f}$ & 1 & $f$ & $-N / \widetilde{N}$ & $2 \widetilde{r}^{\prime}=1-\frac{2 \widetilde{N}}{(K+1) N_{f}}$ \\
$Y$ & $a d j$ & 1 & 1 & 0 & $2 s=\frac{2}{K+1}$ \\
$M_{j}$ & 1 & $f$ & $\bar{f}$ & 0 & $2 r_{M_{j}}=2-\frac{4 N}{(K+1) N_{f}}+\frac{2(j-1)}{K+1}$ \\
\hline
\end{tabular}

Here $j=1, \ldots, K$ and the dual gauge group dimension is

$$
\widetilde{N}=K N_{f}-N, \quad K=1,2, \ldots,
$$

with the constraint $N_{f}>N / K$.

Defining $U=(p q)^{s}=(p q)^{\frac{1}{K+1}}$, we find the following indices for these theories

$$
\begin{aligned}
I_{E}= & \frac{(p ; p)_{\infty}^{N-1}(q ; q)_{\infty}^{N-1}}{N !} \Gamma(U ; p, q)^{N-1} \int_{\mathbb{T}^{N-1}} \prod_{1 \leq i<j \leq N} \frac{\Gamma\left(U z_{i} z_{j}^{-1}, U z_{i}^{-1} z_{j} ; p, q\right)}{\Gamma\left(z_{i} z_{j}^{-1}, z_{i}^{-1} z_{j} ; p, q\right)} \\
& \times \prod_{i=1}^{N_{f}} \prod_{j=1}^{N} \Gamma\left(s_{i} z_{j}, t_{i}^{-1} z_{j}^{-1} ; p, q\right) \prod_{j=1}^{N-1} \frac{d z_{j}}{2 \pi \mathrm{i} z_{j}},
\end{aligned}
$$

where $\prod_{j=1}^{N} z_{j}=1$, the balancing condition reads $U^{2 N} S T^{-1}=(p q)^{N_{f}}$ with $S=\prod_{i=1}^{N_{f}} s_{i}, T=$ $\prod_{i=1}^{N_{f}} t_{i}$, and

$$
\begin{aligned}
I_{M}= & \frac{(p ; p)_{\infty}^{\tilde{N}-1}(q ; q)_{\infty}^{\tilde{N}-1}}{\widetilde{N} !} \Gamma(U ; p, q)^{\widetilde{N}-1} \prod_{l=1}^{K} \prod_{1 \leq i, j \leq N_{f}} \Gamma\left(U^{l-1} s_{i} t_{j}^{-1} ; p, q\right) \\
& \times \int_{T^{\widetilde{N}-1}} \prod_{1 \leq i<j \leq \widetilde{N}} \frac{\Gamma\left(U z_{i} z_{j}^{-1}, U z_{i}^{-1} z_{j} ; p, q\right)}{\Gamma\left(z_{i} z_{j}^{-1}, z_{i}^{-1} z_{j} ; p, q\right)} \\
& \times \prod_{i=1}^{N_{f}} \prod_{j=1}^{\widetilde{N}} \Gamma\left(U(S T)^{\frac{K}{2 N}} s_{i}^{-1} z_{j}, U(S T)^{-\frac{K}{2 \widetilde{N}}} t_{i} z_{j}^{-1} ; p, q\right) \prod_{j=1}^{\widetilde{N}-1} \frac{d z_{j}}{2 \pi \mathrm{i} z_{j}}
\end{aligned}
$$

where $\prod_{j=1}^{\widetilde{N}} z_{j}=1$.

An important fact is that these theories contain matter fields in the adjoint representation of the gauge group. The conjecture that $I_{E}=I_{M}$ (under appropriate contour separability constraints mentioned earlier) represents a new type of elliptic hypergeometric identities, which was not met earlier [89]. Therefore we describe in Appendix D the total ellipticity property hidden behind this identity. In the large $N, N_{f}$ limit (with fixed $N / N_{f}$ ) the equality of $I_{E}$ and $I_{M}$ was confirmed up to a few terms of the corresponding expansion in [26] using the method of $[24]$. 
9.2. Two adjoint matter fields case. This duality was considered by Brodie and Strassler in $[7,8]$. The electric theory is

\begin{tabular}{|c|c|c|c|c|c|}
\hline & $S U(N)$ & $S U\left(N_{f}\right)$ & $S U\left(N_{f}\right)$ & $U(1)_{B}$ & $U(1)_{R}$ \\
\hline$Q$ & $f$ & $f$ & 1 & 1 & $1-\frac{N}{N_{f}(K+1)}$ \\
$\widetilde{Q}$ & $\bar{f}$ & 1 & $\bar{f}$ & -1 & $1-\frac{N}{N_{f}(K+1)}$ \\
$X$ & $a d j$ & 1 & 1 & 0 & $\frac{2}{K_{K}+1}$ \\
$Y$ & $a d j$ & 1 & 1 & 0 & $\frac{K}{K+1}$ \\
\hline
\end{tabular}

The magnetic theory has the following matter field content

\begin{tabular}{|c|c|c|c|c|c|}
\hline & $S U(\widetilde{N})$ & $S U\left(N_{f}\right)$ & $S U\left(N_{f}\right)$ & $U(1)_{B}$ & $U(1)_{R}$ \\
\hline$q$ & $f$ & $\bar{f}$ & 1 & $\frac{N}{N}$ & $1-\frac{\tilde{N}}{N_{f}(K+1)}$ \\
$\widetilde{q}$ & $\bar{f}$ & 1 & $f$ & $-\frac{N}{\widetilde{N}}$ & $1-\frac{\widetilde{N}}{N_{f}(K+1)}$ \\
$X$ & $a d j$ & 1 & 1 & 0 & $\frac{2}{K_{K} 1}$ \\
$Y$ & $a d j$ & 1 & 1 & 0 & $\frac{{ }^{K+1}}{N_{f}}$ \\
$M_{L J}$ & 1 & $f$ & $\bar{f}$ & 0 & $2-\frac{2 N}{N_{f}(K+1)}+\frac{2 L+K J}{K+1}$ \\
\hline
\end{tabular}

Here $K$ is odd, $0 \leq L \leq K-1, J=0,1,2$, and

$$
\widetilde{N}=3 K N_{f}-N \text {. }
$$

Corresponding electric superconformal index has the form

$$
\begin{aligned}
I_{E}=\frac{(p ; p)_{\infty}^{N-1}(q ; q)_{\infty}^{N-1}}{N !} \Gamma\left(U, U^{\frac{K}{2}} ; p, q\right)^{N-1} \\
\quad \times \int_{\mathbb{T}^{N-1}} \prod_{1 \leq i<j \leq N} \frac{\Gamma\left(U z_{i} z_{j}^{-1}, U z_{i}^{-1} z_{j}, U^{K / 2} z_{i} z_{j}^{-1}, U^{K / 2} z_{i}^{-1} z_{j} ; p, q\right)}{\Gamma\left(z_{i} z_{j}^{-1}, z_{i}^{-1} z_{j} ; p, q\right)} \\
\quad \times \prod_{i=1}^{N_{f}} \prod_{j=1}^{N} \Gamma\left(s_{i} z_{j}, t_{i}^{-1} z_{j}^{-1} ; p, q\right) \prod_{j=1}^{N-1} \frac{d z_{j}}{2 \pi \mathrm{i} z_{j}},
\end{aligned}
$$

where $\prod_{j=1}^{N} z_{j}=1, U=(p q)^{\frac{1}{K+1}}$, and the balancing condition reads $U^{N} S T^{-1}=(p q)^{N_{f}}$ with $S=\prod_{i=1}^{N_{f}} s_{i}, T=\prod_{i=1}^{N_{f}} t_{i}$. The magnetic index looks like

$$
\begin{aligned}
I_{M}=\frac{(p ; p)_{\infty}^{N-1}(q ; q)_{\infty}^{N-1}}{N !} \Gamma\left(U, U^{\frac{K}{2}} ; p, q\right)^{\widetilde{N}-1} \prod_{L=0}^{K-1} \prod_{J=0}^{2} \prod_{i, j=1}^{N_{f}} \Gamma\left(U^{L+K J / 2} s_{i} t_{j}^{-1} ; p, q\right) \\
\quad \times \int_{\mathbb{T}^{\tilde{N}-1}} \prod_{1 \leq i<j \leq \widetilde{N}} \frac{\Gamma\left(U z_{i} z_{j}^{-1}, U z_{i}^{-1} z_{j} ; p, q\right) \Gamma\left(U^{K / 2} z_{i} z_{j}^{-1}, U^{K / 2} z_{i}^{-1} z_{j} ; p, q\right)}{\Gamma\left(z_{i} z_{j}^{-1}, z_{i}^{-1} z_{j} ; p, q\right)} \\
\quad \times \prod_{i=1}^{N_{f}} \prod_{j=1}^{\widetilde{N}} \Gamma\left(U^{\frac{2-K}{2}}(S T)^{\frac{3 K}{2 \widetilde{N}}} s_{i}^{-1} z_{j}, U^{\frac{2-K}{2}}(S T)^{-\frac{3 K}{2 N}} t_{i} z_{j}^{-1} ; p, q\right) \prod_{j=1}^{\widetilde{N}-1} \frac{d z_{j}}{2 \pi \mathrm{i} z_{j}},
\end{aligned}
$$

where $\prod_{j=1}^{\widetilde{N}} z_{j}=1$. Again, the conjectured equality $I_{E}=I_{M}$ is a new type of identities requiring a rigorous proof.

9.3. Generalized KS type dualities. These dualities were considered in [42]. 
9.3.1. First pair of dual theories. Electric theory:

\begin{tabular}{|c|c|c|c|c|c|c|}
\hline & $S U(N)$ & $S U\left(N_{f}\right)$ & $S U\left(N_{f}\right)$ & $U(1)$ & $U(1)_{B}$ & $U(1)_{R}$ \\
\hline$Q$ & $f$ & $f$ & 1 & 0 & $\frac{1}{N}$ & $2 r=1-\frac{N+2 K}{(K+1) N_{f}}$ \\
$\widetilde{Q}$ & $\bar{f}$ & 1 & $f$ & 0 & $-\frac{1}{N}$ & $2 r=1-\frac{N+2 K}{(K+1) N_{f}}$ \\
$X$ & $T_{A}$ & 1 & 1 & 1 & $\frac{2}{N}$ & $2 s=\frac{1}{K+1}$ \\
$\widetilde{X}$ & $\bar{T}_{A}$ & 1 & 1 & -1 & $-\frac{2}{N}$ & $2 s=\frac{1}{K+1}$ \\
\hline
\end{tabular}

Magnetic theory:

\begin{tabular}{|c|c|c|c|c|c|c|}
\hline & $S U(\widetilde{N})$ & $S U\left(N_{f}\right)$ & $S U\left(N_{f}\right)$ & $U(1)$ & $U(1)_{B}$ & $U(1)_{R}$ \\
\hline$q$ & $f$ & $\bar{f}$ & 1 & $\frac{K\left(N_{f}-2\right)}{\widetilde{N}}$ & $\frac{1}{\widetilde{N}}$ & $2 r^{\prime}=1-\frac{N+2 K}{(K+1) N_{f}}$ \\
$\widetilde{q}$ & $\bar{f}$ & 1 & $\bar{f}$ & $-\frac{K\left(N_{f}-2\right)}{\widetilde{N}}$ & $-\frac{1}{\widetilde{N}}$ & $2 r^{\prime}=1-\frac{\widetilde{N}+2 K}{(K+1) N_{f}}$ \\
$Y$ & $T_{A}$ & 1 & 1 & $\frac{N-N_{f}}{\widetilde{N}}$ & $\frac{2}{\widetilde{N}}$ & $2 s=\frac{1}{K+1}$ \\
$\widetilde{Y}$ & $\bar{T}_{A}$ & 1 & 1 & $-\frac{N-N_{f}}{\widetilde{N}}$ & $-\frac{2}{\widetilde{N}}$ & $2 s=\frac{1}{K+1}$ \\
$M_{j}$ & 1 & $f$ & $f$ & 0 & 0 & $\frac{\widetilde{N}-N+(2 j+1) N_{f}}{N_{f}(K+1)}$ \\
$P_{r}$ & 1 & $T_{A}$ & 1 & -1 & 0 & $\frac{\widetilde{N}-N+(2 r+2) N_{f}}{N_{f}(K+1)}$ \\
$\widetilde{P}_{r}$ & 1 & 1 & $T_{A}$ & 1 & 0 & $\frac{\widetilde{N}-N+(2 r+2) N_{f}}{N_{f}(K+1)}$ \\
\hline
\end{tabular}

Here $j=0, \ldots, K, r=0, \ldots, K-1$, and

$$
\widetilde{N}=(2 K+1) N_{f}-4 K-N, \quad K=0,1,2, \ldots
$$

The electric index is

$$
\begin{aligned}
I_{E}= & \frac{(p ; p)_{\infty}^{N-1}(q ; q)_{\infty}^{N-1}}{N !} \int_{\mathbb{T}^{N-1}} \prod_{1 \leq i<j \leq N} \frac{\Gamma\left(U z_{i} z_{j}, U^{-1}(p q)^{\frac{1}{K+1}} z_{i}^{-1} z_{j}^{-1} ; p, q\right)}{\Gamma\left(z_{i}^{-1} z_{j}, z_{i} z_{j}^{-1} ; p, q\right)} \\
& \times \prod_{j=1}^{N} \prod_{k=1}^{N_{f}} \Gamma\left(s_{k} z_{j}, t_{k} z_{j}^{-1} ; p, q\right) \prod_{j=1}^{N-1} \frac{d z_{j}}{2 \pi \mathrm{i} z_{j}},
\end{aligned}
$$

where $\prod_{j=1}^{N} z_{j}=1$ and $U$ is an arbitrary parameter. The magnetic index is

$$
\begin{aligned}
I_{M}= & \prod_{j=0}^{K} \prod_{k, l=1}^{N_{f}} \Gamma\left((p q)^{\frac{j}{K+1}} s_{k} t_{l} ; p, q\right) \prod_{r=0}^{K-1} \prod_{1 \leq k<l \leq N_{f}} \Gamma\left(U^{-1}(p q)^{\frac{r+1}{K+1}} s_{k} s_{l}, U(p q)^{\frac{r}{K+1}} t_{k} t_{l} ; p, q\right) \\
& \times \frac{(p ; p)_{\infty}^{\widetilde{N}-1}(q ; q)_{\infty}^{\widetilde{N}-1}}{\widetilde{N} !} \int_{\mathbb{T}^{\widetilde{N}-1}} \prod_{1 \leq i<j \leq \widetilde{N}} \frac{\Gamma\left(\widetilde{U} z_{i} z_{j}, \widetilde{U}^{-1}(p q)^{\frac{1}{K+1}} z_{i}^{-1} z_{j}^{-1} ; p, q\right)}{\Gamma\left(z_{i}^{-1} z_{j}, z_{i} z_{j}^{-1} ; p, q\right)} \\
& \times \prod_{j=1}^{\widetilde{N}} \prod_{k=1}^{N_{f}} \Gamma\left((U \widetilde{U})^{\frac{1}{2}} s_{k}^{-1} z_{j},(U \widetilde{U})^{-\frac{1}{2}}(p q)^{\frac{1}{K+1}} t_{k}^{-1} z_{j}^{-1} ; p, q\right) \prod_{j=1}^{\widetilde{N}-1} \frac{d z_{j}}{2 \pi \mathrm{i} z_{j}}
\end{aligned}
$$

where $\prod_{j=1}^{\widetilde{N}} z_{j}=1$, the balancing condition looks as $S T=(p q)^{N_{f}-\frac{N+2 K}{K+1}}$ with $S=\prod_{j=1}^{N_{f}} s_{j}, T=$ $\prod_{j=1}^{N_{f}} t_{j}$, and $\widetilde{U}=U^{\frac{N-N_{f}}{\tilde{N}}}\left(S T^{-1}\right)^{\frac{1}{N}}(p q)^{\frac{\widetilde{N}-N+N_{f}}{2 \tilde{N}(K+1)}}$. 
9.3.2. Second pair of dual theories. Electric theory:

\begin{tabular}{|c|c|c|c|c|c|c|}
\hline & $S U(N)$ & $S U\left(N_{f}\right)$ & $S U\left(N_{f}\right)$ & $U(1)$ & $U(1)_{B}$ & $U(1)_{R}$ \\
\hline$Q$ & $f$ & $f$ & 1 & 0 & $\frac{1}{N}$ & $2 r=1-\frac{N-2 K}{(K+1) N_{f}}$ \\
$\widetilde{Q}$ & $\bar{f}$ & 1 & $f$ & 0 & $-\frac{1}{N}$ & $2 r=1-\frac{N-2 K}{(K+1) N_{f}}$ \\
$X$ & $T_{S}$ & 1 & 1 & 1 & $\frac{2}{N}$ & $2 s=\frac{1}{K+1}$ \\
$\widetilde{X}$ & $\bar{T}_{S}$ & 1 & 1 & -1 & $-\frac{2}{N}$ & $2 s=\frac{1}{K+1}$ \\
\hline
\end{tabular}

Magnetic theory:

\begin{tabular}{|c|c|c|c|c|c|c|}
\hline & $S U(\widetilde{N})$ & $S U\left(N_{f}\right)$ & $S U\left(N_{f}\right)$ & $U(1)$ & $U(1)_{B}$ & $U(1)_{R}$ \\
\hline$q$ & $f$ & $\bar{f}$ & 1 & $\frac{K\left(N_{f}+2\right)}{\widetilde{N}}$ & $\frac{1}{\widetilde{N}}$ & $2 r^{\prime}=1-\frac{N}{(K+1) N_{f}}$ \\
$\widetilde{q}$ & $\bar{f}$ & 1 & $\bar{f}$ & $-\frac{K\left(N_{f}+2\right)}{\widetilde{N}}$ & $-\frac{1}{\widetilde{N}}$ & $2 r^{\prime}=1-\frac{\widetilde{N}-2 K}{(K+1) N_{f}}$ \\
$Y$ & $T_{S}$ & 1 & 1 & $\frac{N-N_{f}}{\widetilde{N}}$ & $\frac{2}{\widetilde{N}}$ & $2 s=\frac{1}{K+1}$ \\
$\widetilde{Y}$ & $\bar{T}_{S}$ & 1 & 1 & $-\frac{N_{-}-N_{f}}{\widetilde{N}}$ & $-\frac{2}{\widetilde{N}}$ & $2 s=\frac{1}{K+1}$ \\
$M_{j}$ & 1 & $f$ & $f$ & 0 & 0 & $\frac{\widetilde{N}-N+(2 j+1) N_{f}}{N_{f}(K+1)}$ \\
$P_{r}$ & 1 & $T_{S}$ & 1 & -1 & 0 & $\frac{\widetilde{N}-N+(2 r+2) N_{f}}{N_{f}(K+1)}$ \\
$\widetilde{P}_{r}$ & 1 & 1 & $T_{S}$ & 1 & 0 & $\frac{\widetilde{N}-N+(2 r+2) N_{f}}{N_{f}(K+1)}$ \\
\hline
\end{tabular}

Here $j=0, \ldots, K, r=0, \ldots, K-1$, and

$$
\widetilde{N}=(2 K+1) N_{f}+4 K-N, \quad K=0,1,2, \ldots
$$

The electric index is given by the integral

$$
\begin{aligned}
I_{E}= & \frac{(p ; p)_{\infty}^{N-1}(q ; q)_{\infty}^{N-1}}{N !} \int_{\mathbb{T}^{N-1}} \prod_{1 \leq i<j \leq N} \frac{\Gamma\left(U z_{i} z_{j}, U^{-1}(p q)^{\frac{1}{K+1}} z_{i}^{-1} z_{j}^{-1} ; p, q\right)}{\Gamma\left(z_{i}^{-1} z_{j}, z_{i} z_{j}^{-1} ; p, q\right)} \\
& \times \prod_{j=1}^{N} \Gamma\left(U z_{j}^{2}, U^{-1}(p q)^{\frac{1}{K+1}} z_{j}^{-2} ; p, q\right) \prod_{k=1}^{N_{f}} \Gamma\left(s_{k} z_{j}, t_{k} z_{j}^{-1} ; p, q\right) \prod_{j=1}^{N-1} \frac{d z_{j}}{2 \pi \mathrm{i} z_{j}}
\end{aligned}
$$

where $\prod_{j=1}^{N} z_{j}=1$. The magnetic index is

$$
\begin{aligned}
I_{M}= & \prod_{j=0}^{K} \prod_{k, l=1}^{N_{f}} \Gamma\left((p q)^{\frac{j}{K+1}} s_{k} t_{l} ; p, q\right) \prod_{r=0}^{K-1} \prod_{1 \leq k<l \leq N_{f}} \Gamma\left(U^{-1}(p q)^{\frac{r+1}{K+1}} s_{k} s_{l}, U(p q)^{\frac{r}{K+1}} t_{k} t_{l} ; p, q\right) \\
& \times \prod_{r=0}^{K-1} \prod_{k=1}^{N_{f}} \Gamma\left(U^{-1}(p q)^{\frac{r+1}{K+1}} s_{k}^{2}, U(p q)^{\frac{r}{K+1}} t_{k}^{2} ; p, q\right) \frac{(p ; p)_{\infty}^{\widetilde{N}-1}(q ; q)_{\infty}^{\widetilde{N}-1}}{\widetilde{N} !} \\
& \times \int_{\mathbb{T}^{\widetilde{N}}-1} \prod_{1 \leq i<j \leq \widetilde{N}} \frac{\Gamma\left(\widetilde{U} z_{i} z_{j}, \widetilde{U^{-1}}(p q)^{\frac{1}{K+1}} z_{i}^{-1} z_{j}^{-1} ; p, q\right)}{\Gamma\left(z_{i}^{-1} z_{j}, z_{i} z_{j}^{-1} ; p, q\right)} \prod_{j=1}^{\widetilde{N}} \Gamma\left(\widetilde{U} z_{j}^{2}, \widetilde{U}^{-1}(p q)^{\frac{1}{K+1}} z_{j}^{-2} ; p, q\right) \\
& \times \prod_{j=1}^{\widetilde{N}} \prod_{k=1}^{N_{f}} \Gamma\left((U \widetilde{U})^{\frac{1}{2}} s_{k}^{-1} z_{j},(U \widetilde{U})^{-\frac{1}{2}}(p q)^{\frac{1}{K+1}} t_{k}^{-1} z_{j}^{-1} ; p, q\right) \prod_{j=1}^{\widetilde{N}-1} \frac{d z_{j}}{2 \pi i z_{j}}
\end{aligned}
$$


where $\prod_{j=1}^{\widetilde{N}} z_{j}=1$, the balancing condition reads $S T=(p q)^{N_{f}-\frac{N-2 K}{K+1}}$ with $S=\prod_{j=1}^{N_{f}} s_{j}, T=$ $\prod_{j=1}^{N_{f}} t_{j}$, and $\widetilde{U}=U^{\frac{N-N_{f}}{\tilde{N}}}\left(S T^{-1}\right)^{\frac{1}{N}}(p q)^{\frac{\widetilde{N}-N+N_{f}}{2 \tilde{N}(K+1)}}$.

9.3.3. Third pair of dual theories. In comparison with the dualities described in previous two subsections, this case involves non-abelian flavor subgroups of different ranks.

The electric theory:

\begin{tabular}{|c|c|c|c|c|c|c|}
\hline & $S U(N)$ & $S U\left(N_{f}\right)$ & $S U\left(N_{f}-8\right)$ & $U(1)$ & $U(1)_{B}$ & $U(1)_{R}$ \\
\hline$Q$ & $f$ & $f$ & 1 & $-(2 K+1)+\frac{2(4 K+3)}{N_{f}}$ & $\frac{1}{N}$ & $2 r=1-\frac{N+2(4 K+3)}{2(K+1) N_{f}}$ \\
$\widetilde{Q}$ & $\bar{f}$ & 1 & $f$ & $2 K+1+\frac{2(4 K+3)}{N_{f}-8}$ & $-\frac{1}{N}$ & $2 \widetilde{r}=1-\frac{N-2(4 K+3)}{2(K+1)\left(N_{f}-8\right)}$ \\
$X$ & $T_{A}$ & 1 & 1 & 1 & $\frac{2}{N}$ & $2 s=\frac{1}{2(K+1)}$ \\
$\widetilde{X}$ & $\bar{T}_{S}$ & 1 & 1 & -1 & $-\frac{2}{N}$ & $2 s=\frac{1}{2(K+1)}$ \\
\hline
\end{tabular}

The magnetic theory:

\begin{tabular}{|c|c|c|c|c|c|c|}
\hline & $S U(\widetilde{N})$ & $S U\left(N_{f}\right)$ & $S U\left(N_{f}-8\right)$ & $U(1)$ & $U(1)_{B}$ & $U(1)_{R}$ \\
\hline$q$ & $f$ & $\bar{f}$ & 1 & $2 K+1-\frac{2(4 K+3)}{N_{f}}$ & $\frac{1}{\widetilde{N}}$ & $2 r^{\prime}=1-\frac{\tilde{N}+2(4 K+3)}{2(K+1) N_{f}}$ \\
$\widetilde{q}$ & $\bar{f}$ & 1 & $\bar{f}$ & $-2 K-1-\frac{2(4 K+3)}{N_{f}-8}$ & $-\frac{1}{\widetilde{N}}$ & $2 \widetilde{r}^{\prime}=1-\frac{\widetilde{N}-2(4 K+3)}{2(K+1)\left(N_{f}-8\right)}$ \\
$Y$ & $T_{A}$ & 1 & 1 & -1 & $\frac{2}{\widetilde{N}}$ & $2 s=\frac{1}{2(K+1)}$ \\
$\widetilde{Y}$ & $\bar{T}_{S}$ & 1 & 1 & 1 & $-\frac{2}{\widetilde{N}}$ & $2 s=\frac{1}{2(K+1)}$ \\
$M_{J}$ & 1 & $f$ & $f$ & $\frac{2(4 K+3)\left(2 N_{f}-8\right)}{N_{f}\left(N_{f}-8\right)}$ & 0 & $2(r+\widetilde{r})+\frac{J}{K+1}$ \\
$P_{2 L}$ & 1 & $T_{S}$ & 1 & $-4 K-3+\frac{4(4 K+3)}{N_{f}}$ & 0 & $4 r+\frac{4 L+1}{2(K+1)}$ \\
$P_{2 M+1}$ & 1 & $T_{A}$ & 1 & $-4 K-3+\frac{4(4 K+3)}{N_{f}}$ & 0 & $4 r+\frac{4 M+3}{2(K+1)}$ \\
$\widetilde{P}_{2 L}$ & 1 & 1 & $T_{A}$ & $4 K+3+\frac{2(4 K+3)}{N_{f}-8}$ & 0 & $4 \widetilde{r}+\frac{4 L+1}{2(K+1)}$ \\
$\widetilde{P}_{2 M+1}$ & 1 & 1 & $T_{S}$ & $4 K+3+\frac{2(4 K+3)}{N_{f}-8}$ & 0 & $4 \widetilde{r}+\frac{4 M+3}{2(K+1)}$ \\
\hline
\end{tabular}

Here $J=0, \ldots, 2 K+1, L=0, \ldots, K, M=0, \ldots, K-1$, and

$$
\widetilde{N}=(4 K+3)\left(N_{f}-4\right)-N, \quad K=0,1,2, \ldots
$$

The electric index is

$$
\begin{aligned}
I_{E}= & \frac{(p ; p)_{\infty}^{N-1}(q ; q)_{\infty}^{N-1}}{N !} \int_{\mathbb{T}^{N-1}} \prod_{1 \leq i<j \leq N} \frac{\Gamma\left(U z_{i} z_{j}, U^{-1}(p q)^{\frac{1}{2(K+1)}} z_{i}^{-1} z_{j}^{-1} ; p, q\right)}{\Gamma\left(z_{i}^{-1} z_{j}, z_{i} z_{j}^{-1} ; p, q\right)} \\
& \times \prod_{j=1}^{N} \Gamma\left(U^{-1}(p q)^{\frac{1}{2(K+1)}} z_{j}^{-2} ; p, q\right) \prod_{k=1}^{N_{f}} \Gamma\left(s_{k} z_{j} ; p, q\right) \prod_{l=1}^{N_{f}-8} \Gamma\left(t_{l} z_{j}^{-1} ; p, q\right) \prod_{j=1}^{N-1} \frac{d z_{j}}{2 \pi \mathrm{i} z_{j}} .
\end{aligned}
$$

with $\prod_{j=1}^{N} z_{j}=1$ and the balancing condition $S T U^{-4}=(p q)^{N_{f}-4-\frac{N+2}{2(K+1)}}$, where $S=\prod_{j=1}^{N_{f}} s_{j}$, $T=\prod_{j=1}^{N_{f}-8} t_{j}$. The magnetic index is

$$
I_{M}=\frac{(p ; p)_{\infty}^{\tilde{N}-1}(q ; q)_{\infty}^{\tilde{N}-1}}{\widetilde{N} !} \prod_{J=0}^{2 K+1} \prod_{i=1}^{N_{f}} \prod_{j=1}^{N_{f}-8} \Gamma\left((p q)^{\frac{J}{2(K+1)}} s_{i} t_{j} ; p, q\right)
$$


ELLIPTIC HYPERGEOMETRY OF SUPERSYMMETRIC DUALITIES

$$
\begin{aligned}
& \quad \times \prod_{l=0}^{2 K} \prod_{1 \leq i<j \leq N_{f}} \Gamma\left((p q)^{\frac{l+1}{2(K+1)}} U^{-1} s_{i} s_{j} ; p, q\right) \prod_{l=0}^{K} \prod_{i=1}^{N_{f}} \Gamma\left((p q)^{\frac{2 l+1}{2(K+1)}} U^{-1} s_{i}^{2} ; p, q\right) \\
& \quad \times \prod_{m=0}^{2 K} \prod_{1 \leq i<j \leq N_{f}-8} \Gamma\left((p q)^{\frac{m}{2(K+1)}} U t_{i} t_{j} ; p, q\right) \prod_{m=0}^{K-1} \prod_{i=1}^{N_{f}-8} \Gamma\left((p q)^{\frac{2 m+1}{2(K+1)}} U t_{i}^{2} ; p, q\right) \\
& \times \int_{\mathbb{T}^{\tilde{N}-1}} \prod_{1 \leq i<j \leq \widetilde{N}} \frac{\Gamma\left(\widetilde{U} z_{i} z_{j}, \widetilde{U}^{-1}(p q)^{\frac{1}{2(K+1)}} z_{i}^{-1} z_{j}^{-1} ; p, q\right)}{\Gamma\left(z_{i}^{-1} z_{j}, z_{i} z_{j}^{-1} ; p, q\right)} \prod_{j=1}^{\widetilde{N}}\left[\left(\widetilde{U}^{-1}(p q)^{\frac{1}{2(K+1)}} z_{j}^{-2} ; p, q\right)\right. \\
& \left.\quad \times \prod_{k=1}^{N_{f}} \Gamma\left((U \widetilde{U})^{\frac{1}{2}} s_{k}^{-1} z_{j} ; p, q\right) \prod_{l=1}^{N_{f}-8} \Gamma\left((U \widetilde{U})^{-\frac{1}{2}}(p q)^{\frac{1}{2(K+1)}} t_{l}^{-1} z_{j}^{-1} ; p, q\right)\right] \prod_{j=1}^{\widetilde{N}-1} \frac{d z_{j}}{2 \pi \mathrm{i} z_{j}},
\end{aligned}
$$

where $\prod_{j=1}^{\widetilde{N}} z_{j}=1$ and $\widetilde{U}=\left(S^{2} U^{N-N_{f}}\right)^{\frac{1}{N}}$.

9.4. Adjoint, symmetric and conjugate symmetric tensor matter fields. This duality was constructed by Brodie and Strassler [8]. The electric theory is

\begin{tabular}{|c|c|c|c|c|c|c|}
\hline & $S U(N)$ & $S U\left(N_{f}\right)$ & $S U\left(N_{f}\right)$ & $U(1)$ & $U(1)_{B}$ & $U(1)_{R}$ \\
\hline$Q$ & $f$ & $f$ & 1 & 0 & $\frac{1}{N}$ & $1-\frac{N-2}{N_{f}(K+1)}$ \\
$\widetilde{Q}$ & $\bar{f}$ & 1 & $\bar{f}$ & 0 & $-\frac{1}{N}$ & $1-\frac{N-2}{N_{f}(K+1)}$ \\
$X$ & $a d j$ & 1 & 1 & 0 & 0 & $\frac{2}{K+1}$ \\
$Y$ & $T_{S}$ & 1 & 1 & 1 & $\frac{2}{N}$ & $\frac{K}{K+1}$ \\
$\widetilde{Y}$ & $\bar{T}_{S}$ & 1 & 1 & -1 & $-\frac{2}{N}$ & $\frac{K}{K+1}$ \\
\hline
\end{tabular}

The magnetic theory is

\begin{tabular}{|c|c|c|c|c|c|c|}
\hline & $S U(\widetilde{N})$ & $S U\left(N_{f}\right)$ & $S U\left(N_{f}\right)$ & $U(1)$ & $U(1)_{B}$ & $U(1)_{R}$ \\
\hline$q$ & $f$ & $\bar{f}$ & 1 & $\frac{K N_{f}+2}{\widetilde{N}}$ & $\frac{1}{\widetilde{N}}$ & $1-\frac{N-2}{N_{f}(K+1)}$ \\
$\widetilde{q}$ & $\bar{f}$ & 1 & $f$ & $-\frac{K N_{f}+2}{\widetilde{N}}$ & $-\frac{1}{\widetilde{N}}$ & $1-\frac{N-2}{N_{f}(K+1)}$ \\
$X$ & $a d j$ & 1 & 1 & 0 & 0 & $\frac{2}{K+1}$ \\
$Y$ & $T_{S}$ & 1 & 1 & $\frac{N-K N_{f}}{\widetilde{N}}$ & $\frac{2}{\widetilde{N}}$ & $\frac{K}{K+1}$ \\
$\bar{Y}$ & $\bar{T}_{S}$ & 1 & 1 & $-\frac{N-K N_{f}}{\widetilde{N}}$ & $-\frac{2}{N}$ & $\frac{K}{K+1}$ \\
$N_{I}$ & 1 & $f$ & $\bar{f}$ & 0 & 0 & $\frac{2 I}{K+1}+\frac{2 K}{K+1}+2-2 \frac{N-2}{N_{f}(K+1)}$ \\
$M_{I}$ & 1 & $f$ & $\bar{f}$ & 0 & 0 & $\frac{2 I}{K+1}+2-2 \frac{N-2}{N_{f}(K+1)}$ \\
$P_{2 J+1}$ & 1 & $T_{A}$ & 1 & -1 & 0 & $2 \frac{2 J+1}{K+1}+\frac{K}{K+1}+2-2 \frac{N-2}{N_{f}(K+1)}$ \\
$P_{2 J}$ & 1 & $T_{S}$ & 1 & -1 & 0 & $2 \frac{2 J}{K+1}+\frac{K}{K+1}+2-2 \frac{N-2}{N_{f}(K+1)}$ \\
$\widetilde{P}_{2 J+1}$ & 1 & 1 & $\bar{T}_{A}$ & 1 & 0 & $2 \frac{2 J+1}{K+1}+\frac{K}{K+1}+2-2 \frac{N-2}{N_{f}(K+1)}$ \\
$\widetilde{P}_{2 J}$ & 1 & 1 & $\bar{T}_{S}$ & 1 & 0 & $2 \frac{2 J}{K+1}+\frac{K}{K+1}+2-2 \frac{N-2}{N_{f}(K+1)}$ \\
\hline
\end{tabular}

Here $K$ is odd, $I=0,1, \ldots, K-1, J=0,1, \ldots, \frac{K-1}{2}$, but there are no fields $P_{K}, \tilde{P}_{K}$, and

$$
\widetilde{N}=3 K N_{f}+4-N
$$


The indices are

$$
\begin{aligned}
I_{E}= & \frac{(p ; p)_{\infty}^{N-1}(q ; q)_{\infty}^{N-1}}{N !} \Gamma(U ; p, q)^{N-1} \int_{\mathbb{T}^{N-1}} \prod_{1 \leq i<j \leq N} \frac{\Gamma\left(U z_{i} z_{j}^{-1}, U z_{i}^{-1} z_{j} ; p, q\right)}{\Gamma\left(z_{i} z_{j}^{-1}, z_{i}^{-1} z_{j} ; p, q\right)} \\
& \times \prod_{1 \leq i<j \leq N} \Gamma\left(U^{K / 2} X Y z_{i} z_{j}, U^{K / 2}(X Y)^{-1} z_{i}^{-1} z_{j}^{-1} ; p, q\right) \\
& \times \prod_{j=1}^{N}\left[\Gamma\left(U^{K / 2} X Y z_{j}^{2}, U^{K / 2}(X Y)^{-1} z_{j}^{-2} ; p, q\right) \prod_{i=1}^{N_{f}} \Gamma\left(s_{i} z_{j}, t_{i}^{-1} z_{j}^{-1} ; p, q\right)\right] \prod_{j=1}^{N-1} \frac{d z_{j}}{2 \pi \mathrm{i} z_{j}}
\end{aligned}
$$

where $U=(p q)^{\frac{1}{K+1}}, \prod_{j=1}^{N} z_{j}=1$, and

$$
\begin{aligned}
I_{M}= & \frac{(p ; p)_{\infty}^{\widetilde{N}-1}(q ; q)_{\infty}^{\widetilde{N}-1}}{\widetilde{N} !} \Gamma(U ; p, q)^{\widetilde{N}-1} \prod_{L=0}^{K-1} \prod_{i, j=1}^{N_{f}} \Gamma\left(U^{L+K} s_{i} t_{j}^{-1}, U^{L} s_{i} t_{j}^{-1} ; p, q\right) \\
& \times \prod_{J=0}^{K-1} \prod_{1 \leq i<j \leq N_{f}} \Gamma\left((X Y)^{-1} U^{J+K / 2} s_{i} s_{j}, X Y U^{J+K / 2} t_{i}^{-1} t_{j}^{-1} ; p, q\right) \\
\times & \prod_{J=0}^{\frac{K-1}{2}} \prod_{i=1}^{N_{f}} \Gamma\left((X Y)^{-1} U^{2 J+\frac{K}{2}} s_{i}^{2}, X Y U^{2 J+\frac{K}{2}} t_{i}^{-2} ; p, q\right) \int_{\mathbb{T}^{\widetilde{N}-1}} \prod_{1 \leq i<j \leq \widetilde{N}} \frac{\Gamma\left(U z_{i} z_{j}^{-1}, U z_{i}^{-1} z_{j} ; p, q\right)}{\Gamma\left(z_{i} z_{j}^{-1}, z_{i}^{-1} z_{j} ; p, q\right)} \\
& \times \prod_{1 \leq i<j \leq \widetilde{N}} \Gamma\left(U^{K / 2} X^{\frac{N-K N_{f}}{\tilde{N}}} Y^{\frac{N}{N}} z_{i} z_{j}, U^{K / 2}\left(X^{\frac{N-K N_{f}}{\tilde{N}}} Y^{\frac{N}{N}}\right)^{-1} z_{i}^{-1} z_{j}^{-1} ; p, q\right) \\
& \times \prod_{j=1}^{\widetilde{N}} \Gamma\left(U^{K / 2} X^{\frac{N-K N_{f}}{\tilde{N}}} Y^{\frac{N}{N}} z_{j}^{2}, U^{K / 2}\left(X^{\frac{N-K N_{f}}{\tilde{N}}} Y^{\frac{N}{N}}\right)^{-1} z_{j}^{-2} ; p, q\right) \\
\times & \left.\prod_{i=1}^{N_{f}} \Gamma\left(U^{\frac{2-K}{2}} X^{\frac{K N_{f}+2}{\tilde{N}}} Y^{\frac{3 K N_{f}+4}{2 N}} s_{i}^{-1} z_{j}, U^{\frac{2-K}{2}} X^{-\frac{K N_{f}+2}{N}} Y^{-\frac{3 K N_{f}+4}{2 N}} t_{i} z_{j}^{-1} ; p, q\right)\right] \prod_{j=1} \frac{d z_{j}}{2 \pi \mathrm{i} z_{j}},
\end{aligned}
$$

where $Y=(S T)^{1 / N_{f}}, S=\prod_{i=1}^{N_{f}} s_{i}, T=\prod_{i=1}^{N_{f}} t_{i}, X$ is an arbitrary chemical potential associated with the $U(1)$-group, and the balancing condition reads $U^{N-2} S T^{-1}=(p q)^{N_{f}}$.

9.5. Adjoint, anti-symmetric and conjugate anti-symmetric tensor matter fields. This duality was considered in [8]. The electric theory is

\begin{tabular}{|c|c|c|c|c|c|c|}
\hline & $S U(N)$ & $S U\left(N_{f}\right)$ & $S U\left(N_{f}\right)$ & $U(1)$ & $U(1)_{B}$ & $U(1)_{R}$ \\
\hline$Q$ & $f$ & $f$ & 1 & 0 & $\frac{1}{N}$ & $1-\frac{N+2}{N_{f}(K+1)}$ \\
$\widetilde{Q}$ & $\bar{f}$ & 1 & $\bar{f}$ & 0 & $-\frac{1}{N}$ & $1-\frac{N+2}{N_{f}(K+1)}$ \\
$X$ & $a d j$ & 1 & 1 & 0 & 0 & $\frac{2}{K+1}$ \\
$Y$ & $T_{A}$ & 1 & 1 & 1 & $\frac{2}{N}$ & $\frac{K}{K+1}$ \\
$\widetilde{Y}$ & $\bar{T}_{A}$ & 1 & 1 & -1 & $-\frac{2}{N}$ & $\frac{K}{K+1}$ \\
\hline
\end{tabular}

The magnetic theory is 


\begin{tabular}{|c|c|c|c|c|c|c|}
\hline & $S U(\widetilde{N})$ & $S U\left(N_{f}\right)$ & $S U\left(N_{f}\right)$ & $U(1)$ & $U(1)_{B}$ & $U(1)_{R}$ \\
\hline$q$ & $f$ & $\bar{f}$ & 1 & $\frac{K N_{f}-2}{\widetilde{N}}$ & $\frac{1}{\widetilde{N}}$ & $1-\frac{N}{N_{f}(K+1)}$ \\
$\widetilde{q}$ & $\bar{f}$ & 1 & $f$ & $-\frac{K N_{f}-2}{\widetilde{N}}$ & $-\frac{1}{\widetilde{N}}$ & $1-\frac{\tilde{N}+2}{N_{f}(K+1)}$ \\
$X$ & $a d j$ & 1 & 1 & 0 & 0 & $\frac{2}{K+1}$ \\
$Y$ & $T_{A}$ & 1 & 1 & $\frac{N-K N_{f}}{\widetilde{N}_{K N}}$ & $\frac{2}{\widetilde{N}}$ & $\frac{K}{K+1}$ \\
$\widetilde{Y}$ & $\bar{T}_{A}$ & 1 & 1 & $-\frac{N_{f}}{\widetilde{N}}$ & $-\frac{2}{\widetilde{N}}$ & $\frac{K}{K+1}$ \\
$N_{I}$ & 1 & $f$ & $\bar{f}$ & 0 & 0 & $\frac{2 I}{K+1}+\frac{2 K}{K+1}+2-2 \frac{N+2}{N_{f}(K+1)}$ \\
$M_{I}$ & 1 & $f$ & $\bar{f}$ & 0 & 0 & $\frac{2 I}{K+1}+2-2 \frac{N+2}{N_{f}(K+1)}$ \\
$P_{2 J+1}$ & 1 & $T_{S}$ & 1 & -1 & 0 & $2 \frac{2 J+1}{K+1}+\frac{K}{K+1}+2-2 \frac{N+2}{N_{f}(K+1)}$ \\
$P_{2 J}$ & 1 & $T_{A}$ & 1 & -1 & 0 & $2 \frac{2 J}{K+1}+\frac{K}{K+1}+2-2 \frac{N+2}{N_{f}(K+1)}$ \\
$\widetilde{P}_{2 J+1}$ & 1 & 1 & $\bar{T}_{S}$ & 1 & 0 & $2 \frac{2 J+1}{K+1}+\frac{K}{K+1}+2-2 \frac{N+2}{N_{f}(K+1)}$ \\
$\widetilde{P}_{2 J}$ & 1 & 1 & $\bar{T}_{A}$ & 1 & 0 & $2 \frac{2 J}{K+1}+\frac{K}{K+1}+2-2 \frac{N+2}{N_{f}(K+1)}$ \\
\hline
\end{tabular}

Here $K$ is odd, $I=0, \ldots, K-1, J=0, \ldots, \frac{K-1}{2}$, but there are no fields $P_{K}, \tilde{P}_{K}$, and

$$
\widetilde{N}=3 K N_{f}-4-N
$$

The superconformal indices are

$$
\begin{aligned}
I_{E} & =\frac{(p ; p)_{\infty}^{N-1}(q ; q)_{\infty}^{N-1}}{N !} \Gamma(U ; p, q)^{N-1} \int_{\mathbb{T}^{N-1}} \prod_{1 \leq i<j \leq N} \frac{\Gamma\left(U z_{i} z_{j}^{-1}, U z_{i}^{-1} z_{j} ; p, q\right)}{\Gamma\left(z_{i} z_{j}^{-1}, z_{i}^{-1} z_{j} ; p, q\right)} \\
\times & \prod_{1 \leq i<j \leq N} \Gamma\left(U^{K / 2} X Y z_{i} z_{j}, U^{K / 2}(X Y)^{-1} z_{i}^{-1} z_{j}^{-1} ; p, q\right) \prod_{i=1}^{N_{f}} \prod_{j=1}^{N} \Gamma\left(s_{i} z_{j}, t_{i}^{-1} z_{j}^{-1} ; p, q\right) \prod_{j=1}^{N-1} \frac{d z_{j}}{2 \pi \mathrm{i} z_{j}}
\end{aligned}
$$

for $U=(p q)^{\frac{1}{K+1}}, \prod_{j=1}^{N} z_{j}=1$, and

$$
\begin{aligned}
& I_{M}=\frac{(p ; p)_{\infty}^{\widetilde{N}-1}(q ; q)_{\infty}^{\widetilde{N}-1}}{\widetilde{N} !} \Gamma(U ; p, q)^{\widetilde{N}-1} \prod_{L=0}^{K-1} \prod_{i, j=1}^{N_{f}} \Gamma\left(U^{L+K} s_{i} t_{j}^{-1}, U^{L} s_{i} t_{j}^{-1} ; p, q\right) \\
& \times \prod_{J=0}^{K-1} \prod_{1 \leq i<j \leq N_{f}} \Gamma\left((X Y)^{-1} U^{J+K / 2} s_{i} s_{j}, X Y U^{J+K / 2} t_{i}^{-1} t_{j}^{-1} ; p, q\right) \\
& \times \prod_{J=0}^{\frac{K-3}{2}} \prod_{i=1}^{N_{f}} \Gamma\left((X Y)^{-1} U^{2 J+1+\frac{K}{2}} s_{i}^{2}, X Y U^{2 J+1+\frac{K}{2}} t_{i}^{-2} ; p, q\right) \int_{\mathbb{T}^{\tilde{N}-1}} \prod_{1 \leq i<j \leq \widetilde{N}} \frac{\Gamma\left(U z_{i} z_{j}^{-1}, U z_{i}^{-1} z_{j} ; p, q\right)}{\Gamma\left(z_{i} z_{j}^{-1}, z_{i}^{-1} z_{j} ; p, q\right)} \\
& \times \prod_{1 \leq i<j \leq \widetilde{N}} \Gamma\left(U^{K / 2} X^{\frac{N-K N_{f}}{\tilde{N}}} Y^{\frac{N}{N}} z_{i} z_{j}, U^{K / 2}\left(X^{\frac{N-K N_{f}}{\tilde{N}}} Y^{\frac{N}{N}}\right)^{-1} z_{i}^{-1} z_{j}^{-1} ; p, q\right) \\
& \times \prod_{i=1}^{N_{f}} \prod_{j=1}^{\tilde{N}} \Gamma\left(U^{\frac{2-K}{2}} X^{\frac{K N_{f}-2}{\tilde{N}}} Y^{\frac{3 K N_{f}-4}{2 \tilde{N}}} s_{i}^{-1} z_{j}, U^{\frac{2-K}{2}} X^{-\frac{K N_{f}-2}{\tilde{N}}} Y^{-\frac{3 K N_{f}-4}{2 \tilde{N}}} t_{i} z_{j}^{-1} ; p, q\right) \prod_{j=1}^{\tilde{N}-1} \frac{d z_{j}}{2 \pi \mathrm{i} z_{j}}
\end{aligned}
$$

where $\prod_{j=1}^{\widetilde{N}} z_{j}=1, Y=(S T)^{1 / N_{f}}, S=\prod_{i=1}^{N_{f}} s_{i}, T=\prod_{i=1}^{N_{f}} t_{i}, X$ is an arbitrary parameter and the balancing condition reads $U^{N+2} S T^{-1}=(p q)^{N_{f}}$. 
9.6. Adjoint, anti-symmetric and conjugate symmetric tensor matter fields. This duality was discussed by Brodie in [8]. The electric theory is

\begin{tabular}{|c|c|c|c|c|c|c|}
\hline & $S U(N)$ & $S U\left(N_{f}\right)$ & $S U\left(N_{f}-8\right)$ & $U(1)$ & $U(1)_{B}$ & $U(1)_{R}$ \\
\hline$Q$ & $f$ & $f$ & 1 & $x_{1}=\frac{6}{N_{f}}-1$ & $\frac{1}{N}$ & $2 r_{1}=1-\frac{N+6 K}{N_{f}(K+1)}$ \\
$\widetilde{Q}$ & $\bar{f}$ & 1 & $f$ & $x_{2}=\frac{6}{N_{f}-8}+1$ & $-\frac{1}{N}$ & $2 r_{2}=1-\frac{N-6 K}{\left(N_{f}-8\right)(K+1)}$ \\
$X$ & $a d j$ & 1 & 1 & 0 & 0 & $\frac{2}{K_{K} 1}$ \\
$Y$ & $T_{A}$ & 1 & 1 & 1 & $\frac{2}{N}$ & $\frac{K}{K+1}$ \\
$\widetilde{Y}$ & $\bar{T}_{S}$ & 1 & 1 & -1 & $-\frac{2}{N}$ & $\frac{K}{K+1}$ \\
\hline
\end{tabular}

In the original paper [8] there were misprints for the values of $U(1)$-group hypercharges which were corrected in [50]. The magnetic theory is

\begin{tabular}{|c|c|c|c|c|c|c|}
\hline & $S U(\widetilde{N})$ & $S U\left(N_{f}\right)$ & $S U\left(N_{f}-8\right)$ & $U(1)$ & $U(1)_{B}$ & $U(1)_{R}$ \\
\hline$q$ & $f$ & $\bar{f}$ & 1 & $1-\frac{6}{N_{f}}$ & $\frac{1}{\widetilde{N}}$ & $1-\frac{\tilde{N}+6 K}{N_{f}(K+1)}$ \\
$\widetilde{q}$ & $\bar{f}$ & 1 & $\bar{f}$ & $-1-\frac{6}{N_{f}-8}$ & $-\frac{1}{\widetilde{N}}$ & $1-\frac{\tilde{N}-6 K}{\left(N_{f}-8\right)(K+1)}$ \\
$X$ & $a d j$ & 1 & 1 & 0 & 0 & $\frac{2}{K+1}$ \\
$Y$ & $T_{A}$ & 1 & 1 & -1 & $\frac{2}{\widetilde{N}}$ & $\frac{K}{K+1}$ \\
$\widetilde{Y}$ & $\bar{T}_{S}$ & 1 & 1 & 1 & $-\frac{2}{\widetilde{N}}$ & $\frac{K}{K+1}$ \\
$N_{J}$ & 1 & $f$ & $f$ & $x_{1}+x_{2}$ & 0 & $\frac{2 J}{K+1}+\frac{2 K}{K+1}+2 r_{1}+2 r_{2}$ \\
$M_{J}$ & 1 & $f$ & $f$ & $x_{1}+x_{2}$ & 0 & $\frac{2 J}{K+1}+2 r_{1}+2 r_{2}$ \\
$P_{J}$ & 1 & $T_{S}$ & 1 & $2 x_{1}-1$ & 0 & $\frac{2 J}{K+1}+\frac{K}{K+1}+2-2 \frac{\widetilde{N}+6 K}{N_{f}(K+1)}$ \\
$\widetilde{P}_{J}$ & 1 & 1 & $T_{A}$ & $2 x_{2}+1$ & 0 & $\frac{2 J}{K+1}+\frac{K}{K+1}+2-2 \frac{N-6 K}{\left(N_{f}-8\right)(K+1)}$ \\
\hline
\end{tabular}

Here $J=0,1, \ldots, K-1$ and

$$
\widetilde{N}=3 K\left(N_{f}-4\right)-N
$$

The superconformal indices are

$$
\begin{aligned}
& I_{E}=\frac{(p ; p)_{\infty}^{N-1}(q ; q)_{\infty}^{N-1}}{N !} \Gamma(U ; p, q)^{N-1} \int_{\mathbb{T}^{N-1}} \prod_{1 \leq i<j \leq N} \frac{\Gamma\left(U z_{i} z_{j}^{-1}, U z_{i}^{-1} z_{j} ; p, q\right)}{\Gamma\left(z_{i} z_{j}^{-1}, z_{i}^{-1} z_{j} ; p, q\right)} \\
& \prod_{1 \leq i<j \leq N} \Gamma\left(U^{K / 2} X Y z_{i} z_{j}, U^{K / 2}(X Y)^{-1} z_{i}^{-1} z_{j}^{-1} ; p, q\right) \\
& \times \prod_{i=1}^{N} \Gamma\left(U^{K / 2}(X Y)^{-1} z_{i}^{-2} ; p, q\right) \prod_{j=1}^{N} \prod_{i=1}^{N_{f}} \Gamma\left(s_{i} z_{j} ; p, q\right) \prod_{k=1}^{N_{f}-8} \Gamma\left(t_{k} z_{j}^{-1} ; p, q\right) \prod_{j=1}^{N-1} \frac{d z_{j}}{2 \pi \mathrm{i} z_{j}}
\end{aligned}
$$


for $U=(p q)^{\frac{1}{K+1}}, \prod_{j=1}^{N} z_{j}=1$, and

$$
\begin{aligned}
I_{M}= & \frac{(p ; p)_{\infty}^{\tilde{N}-1}(q ; q)_{\infty}^{\tilde{N}-1}}{\widetilde{N} !} \Gamma(U ; p, q)^{\tilde{N}-1} \prod_{L=0}^{K-1} \prod_{i=1}^{N_{f}} \prod_{j=1}^{N_{f}-8} \Gamma\left(U^{L+K} s_{i} t_{j}, U^{L} s_{i} t_{j} ; p, q\right) \\
& \times \prod_{J=0}^{K-1} \prod_{1 \leq i<j \leq N_{f}} \Gamma\left((X Y)^{-1} U^{J+K / 2} s_{i} s_{j} ; p, q\right) \prod_{1 \leq i<j \leq N_{f}-8} \Gamma\left(X Y U^{J+K / 2} t_{i} t_{j} ; p, q\right) \\
& \times \prod_{J=0}^{K-1} \prod_{i=1}^{N_{f}} \Gamma\left((X Y)^{-1} U^{J+K / 2} s_{i}^{2} ; p, q\right) \int_{\mathbb{T}^{\tilde{N}-1}} \prod_{1 \leq i<j \leq \widetilde{N}} \frac{\Gamma\left(U z_{i} z_{j}^{-1}, U z_{i}^{-1} z_{j} ; p, q\right)}{\Gamma\left(z_{i} z_{j}^{-1}, z_{i}^{-1} z_{j} ; p, q\right)} \\
& \times \prod_{1 \leq i<j \leq \widetilde{N}} \Gamma\left(U^{K / 2} X^{-1} Y^{\frac{N}{N}} z_{i} z_{j}, U^{K / 2} X Y^{-\frac{N}{N}} z_{i}^{-1} z_{j}^{-1} ; p, q\right) \\
& \times \prod_{i=1}^{\tilde{N}} \Gamma\left(U^{K / 2} X Y^{-\frac{N}{N}} z_{i}^{-2} ; p, q\right) \prod_{j=1}^{\widetilde{N}} \prod_{i=1}^{N_{f}} \Gamma\left(U^{\frac{2-K}{2}} Y^{\frac{3 K\left(N_{f}-4\right)}{2 \tilde{N}}} s_{i}^{-1} z_{j} ; p, q\right) \\
& \times \prod_{j=1}^{\widetilde{N}} \prod_{k=1}^{N_{f}-8} \Gamma\left(U^{\frac{2-K}{2}} Y^{-\frac{3 K\left(N_{f}-4\right)}{2 \tilde{N}}} t_{k}^{-1} z_{j}^{-1} ; p, q\right) \prod_{j=1}^{\widetilde{N}-1} \frac{d z_{j}}{2 \pi \mathrm{i} z_{j}},
\end{aligned}
$$

where $\prod_{j=1}^{\widetilde{N}} z_{j}=1, Y=\left(S T^{-1} X^{2 N_{f}-8}(p q)^{\frac{2(K-2)}{K+1}}\right)^{\frac{1}{N_{f}-4}}$, and the balancing condition reads $U^{N} X^{-4} Y^{-4} S T=(p q)^{N_{f}-4}$ with $S=\prod_{i=1}^{N_{f}} s_{i}, T=\prod_{i=1}^{N_{f}-8} t_{i}$.

The equalities $I_{E}=I_{M}$ for all the dualities described in this section require a rigorous mathematical confirmation. For the moment we have only one justifying argument coming from the total ellipticity condition associated with the kernels of the corresponding pairs of integrals.

\section{KS TYPE DUALITIES FOR SYMPLECTIC GAUGE GROUPS}

10.1. The anti-symmetric tensor matter field. For $S P(2 N)$ group the following electricmagnetic duality was discovered by Intriligator in [41]. The electric theory:

\begin{tabular}{|c|c|c|c|}
\hline & $S P(2 N)$ & $S U\left(2 N_{f}\right)$ & $U(1)_{R}$ \\
\hline$Q$ & $f$ & $f$ & $2 r=1-\frac{2(N+K)}{(K+1) N_{f}}$ \\
$X$ & $T_{A}$ & 1 & $2 s=\frac{2}{K+1}$ \\
\hline
\end{tabular}

The magnetic theory:

\begin{tabular}{|c|c|c|c|}
\hline & $S P(2 \widetilde{N})$ & $S U\left(2 N_{f}\right)$ & $U(1)_{R}$ \\
\hline$q$ & $\mathrm{f}$ & $\bar{f}$ & $2 \widetilde{r}=1-\frac{2(\tilde{N}+K)}{(K+1) N_{f}}$ \\
$Y$ & $T_{A}$ & 1 & $2 s=\frac{2}{K+1}$ \\
$M_{j}$ & 1 & $T_{A}$ & $2 r_{j}=2 \frac{K+j}{K+1}-4 \frac{\widetilde{N}+K}{(K+1) N_{f}}$ \\
\hline
\end{tabular}

where $j=1, \ldots, K$, and

$$
\widetilde{N}=K\left(N_{f}-2\right)-N, \quad K=1,2, \ldots
$$

Defining $U=(p q)^{s}=(p q)^{\frac{1}{K+1}}$, we find the following indices for these theories 


$$
\begin{aligned}
I_{E}= & \frac{(p ; p)_{\infty}^{N}(q ; q)_{\infty}^{N}}{2^{N} N !} \Gamma(U ; p, q)^{N-1} \int_{\mathbb{T}^{N}} \prod_{1 \leq i<j \leq N} \frac{\Gamma\left(U z_{i}^{ \pm 1} z_{j}^{ \pm 1} ; p, q\right)}{\Gamma\left(z_{i}^{ \pm 1} z_{j}^{ \pm 1} ; p, q\right)} \\
& \times \prod_{j=1}^{N} \frac{\prod_{i=1}^{2 N_{f}} \Gamma\left(s_{i} z_{j}^{ \pm 1} ; p, q\right)}{\Gamma\left(z_{j}^{ \pm 2} ; p, q\right)} \prod_{j=1}^{N} \frac{d z_{j}}{2 \pi \mathrm{i} z_{j}}
\end{aligned}
$$

and

$$
\begin{aligned}
I_{M}= & \frac{(p ; p)_{\infty}^{\widetilde{N}}(q ; q)_{\infty}^{\widetilde{N}}}{2^{\widetilde{N}} \widetilde{N} !} \Gamma(U ; p, q)^{\widetilde{N}-1} \prod_{l=1}^{K} \prod_{1 \leq i<j \leq 2 N_{f}} \Gamma\left(U^{l-1} s_{i} s_{j} ; p, q\right) \\
& \times \int_{\mathbb{T}^{\widetilde{N}}} \prod_{1 \leq i<j \leq \widetilde{N}} \frac{\Gamma\left(U z_{i}^{ \pm 1} z_{j}^{ \pm 1} ; p, q\right)}{\Gamma\left(z_{i}^{ \pm 1} z_{j}^{ \pm 1} ; p, q\right)} \prod_{j=1}^{\widetilde{N}} \frac{\prod_{i=1}^{2 N_{f}} \Gamma\left(U s_{i}^{-1} z_{j}^{ \pm 1} ; p, q\right)}{\Gamma\left(z_{j}^{ \pm 2} ; p, q\right)} \prod_{j=1}^{\widetilde{N}} \frac{d z_{j}}{2 \pi \mathrm{i} z_{j}}
\end{aligned}
$$

where the balancing condition reads $U^{2(N+K)} \prod_{i=1}^{2 N_{f}} s_{i}=(p q)^{N_{f}}$.

10.2. Symmetric tensor matter field. Another electric-magnetic duality is described by Leigh and Strassler in [54]. The electric theory:

\begin{tabular}{|c|c|c|c|}
\hline & $S P(2 N)$ & $S U\left(2 N_{f}\right)$ & $U(1)_{R}$ \\
\hline$Q$ & $f$ & $f$ & $2 r=1-\frac{N+1}{(K+1) N_{f}}$ \\
$X$ & $a d j=T_{S}$ & 1 & $2 s=\frac{1}{K+1}$ \\
\hline
\end{tabular}

The magnetic theory:

\begin{tabular}{|c|c|c|c|}
\hline & $S P(2 \widetilde{N})$ & $S U\left(2 N_{f}\right)$ & $U(1)_{R}$ \\
\hline$q$ & $f$ & $\bar{f}$ & $2 \widetilde{r}=1-\frac{\tilde{N}+1}{(K+1) N_{f}}$ \\
$Y$ & $a d j$ & 1 & $2 s=\frac{1}{K+1}$ \\
$M_{2 j}, j=0, \ldots, K$ & 1 & $T_{A}$ & $2 r_{2 j}=2-\frac{2(N+1)-2 j N_{f}}{(K+1) N_{f}}$ \\
$M_{2 j+1}, j=0, \ldots, K-1$ & 1 & $T_{S}$ & $2 r_{2 j+1}=2-\frac{2(N+1)-(2 j+1) N_{f}}{(K+1) N_{f}}$ \\
\hline
\end{tabular}

Here

$$
\widetilde{N}=(2 K+1) N_{f}-N-2, \quad K=0,1,2, \ldots
$$

Defining $U=(p q)^{s}=(p q)^{\frac{1}{2(K+1)}}$, we find the following superconformal indices

$$
\begin{aligned}
I_{E}= & \frac{(p ; p)_{\infty}^{N}(q ; q)_{\infty}^{N}}{2^{N} N !} \Gamma(U ; p, q)^{N} \int_{\mathbb{T}^{N}} \prod_{1 \leq i<j \leq N} \frac{\Gamma\left(U z_{i}^{ \pm 1} z_{j}^{ \pm 1} ; p, q\right)}{\Gamma\left(z_{i}^{ \pm 1} z_{j}^{ \pm 1} ; p, q\right)} \\
& \times \prod_{j=1}^{N} \frac{\Gamma\left(U z_{j}^{ \pm 2} ; p, q\right)}{\Gamma\left(z_{j}^{ \pm 2} ; p, q\right)} \prod_{i=1}^{2 N_{f}} \prod_{j=1}^{N} \Gamma\left(s_{i} z_{j}^{ \pm 1} ; p, q\right) \prod_{j=1}^{N} \frac{d z_{j}}{2 \pi \mathrm{i} z_{j}}
\end{aligned}
$$


and

$$
\begin{aligned}
I_{M}= & \frac{(p ; p)_{\infty}^{\widetilde{N}}(q ; q)_{\infty}^{\widetilde{N}}}{2^{\widetilde{N}} \widetilde{N} !} \Gamma(U ; p, q)^{\widetilde{N}} \prod_{l=0}^{2 K} \prod_{1 \leq i<j \leq 2 N_{f}} \Gamma\left(U^{l} s_{i} s_{j} ; p, q\right) \\
& \times \prod_{l=0}^{K-1} \prod_{i=1}^{2 N_{f}} \Gamma\left(U^{2 l+1} s_{i}^{2} ; p, q\right) \int_{\mathbb{T}^{\tilde{N}}} \prod_{1 \leq i<j \leq \widetilde{N}} \frac{\Gamma\left(U z_{i}^{ \pm 1} z_{j}^{ \pm 1} ; p, q\right)}{\Gamma\left(z_{i}^{ \pm 1} z_{j}^{ \pm 1} ; p, q\right)} \\
& \times \prod_{j=1}^{\widetilde{N}} \frac{\Gamma\left(U z_{j}^{ \pm 2} ; p, q\right)}{\Gamma\left(z_{j}^{ \pm 2} ; p, q\right)} \prod_{i=1}^{2 N_{f}} \prod_{j=1}^{\widetilde{N}} \Gamma\left(U s_{i}^{-1} z_{j}^{ \pm 1} ; p, q\right) \prod_{j=1}^{\widetilde{N}} \frac{d z_{j}}{2 \pi \mathrm{i} z_{j}}
\end{aligned}
$$

where the balancing condition reads $U^{2(N+1)} \prod_{i=1}^{2 N_{f}} s_{i}=(p q)^{N_{f}}$.

10.3. Two anti-symmetric tensor matter fields. This duality was investigated by Brodie and Strassler in [8]. The electric theory:

\begin{tabular}{|c|c|c|c|}
\hline & $S P(2 N)$ & $S U\left(2 N_{f}\right)$ & $U(1)_{R}$ \\
\hline$Q$ & $f$ & $f$ & $1-\frac{N+2 K+1}{(K+1) N_{f}}$ \\
$X$ & $T_{A}$ & 1 & $\frac{2}{K_{K} 1}$ \\
$Y$ & $T_{A}$ & 1 & $\frac{K}{K+1}$ \\
\hline
\end{tabular}

The magnetic theory:

\begin{tabular}{|c|c|c|c|}
\hline & $S P(2 \tilde{N})$ & $S U\left(2 N_{f}\right)$ & $U(1)_{R}$ \\
\hline$q$ & $f$ & $\bar{f}$ & $1-\frac{\tilde{N}+2 K+1}{(K+1) N_{f}}$ \\
$\widetilde{X}$ & $T_{A}$ & 1 & $\frac{2}{K+1}$ \\
$\widetilde{Y}$ & $T_{A}$ & 1 & $\frac{K}{K+1}$ \\
$M_{J 0}, J=0, \ldots, K-1$ & 1 & $T_{A}$ & $2-\frac{N+2 K+1}{(K+1) N_{f}}+\frac{2 J}{K+1}$ \\
$M_{2 J 1}, J=0, \ldots, \frac{K-1}{2}$ & 1 & $T_{A}$ & $2-\frac{N+2 K+1}{(K+1) N_{f}}+\frac{2(2 J)}{K+1}+\frac{K}{K+1}$ \\
$M_{2 J+1}, J=0, \ldots, \frac{K-3}{2}$ & 1 & $T_{S}$ & $2-\frac{N+2 K+1}{(K+1) N_{f}}+\frac{2(2 J+1)}{K+1}+\frac{K}{K+1}$ \\
$M_{J 2}, J=0, \ldots, K-1$ & 1 & $T_{A}$ & $2-\frac{N+2 K+1}{(K+1) N_{f}}+\frac{2 J}{K+1}+\frac{2 K}{K+1}$ \\
\hline
\end{tabular}

Here $K$ is odd and

$$
\widetilde{N}=3 K N_{f}-4 K-2-N
$$

For these theories we have the following superconformal indices

$$
\begin{aligned}
I_{E}= & \frac{(p ; p)_{\infty}^{N}(q ; q)_{\infty}^{N}}{2^{N} N !} \Gamma\left(U, U^{\frac{K}{2}} ; p, q\right)^{N-1} \\
& \times \int_{\mathbb{T}^{N}} \prod_{1 \leq i<j \leq N} \frac{\Gamma\left(U z_{i}^{ \pm 1} z_{j}^{ \pm 1}, U^{\frac{K}{2}} z_{i}^{ \pm 1} z_{j}^{ \pm 1} ; p, q\right)}{\Gamma\left(z_{i}^{ \pm 1} z_{j}^{ \pm 1} ; p, q\right)} \prod_{i=1}^{2 N_{f}} \prod_{j=1}^{N} \frac{\Gamma\left(s_{i} z_{j}^{ \pm 1} ; p, q\right)}{\Gamma\left(z_{j}^{ \pm 2} ; p, q\right)} \prod_{j=1}^{N} \frac{d z_{j}}{2 \pi \mathrm{i} z_{j}}
\end{aligned}
$$


where $U=(p q)^{\frac{1}{K+1}}$, the balancing condition reads $U^{N+2 K+1} \prod_{i=1}^{2 N_{f}} s_{i}=(p q)^{N_{f}}$, and

$$
\begin{aligned}
I_{M}= & \frac{(p ; p)_{\infty}^{\widetilde{N}}(q ; q)_{\infty}^{\widetilde{N}}}{2^{\widetilde{N}} \widetilde{N} !} \Gamma\left(U, U^{\frac{K}{2}} ; p, q\right)^{\widetilde{N}-1} \\
& \times \prod_{J=0}^{K-1} \prod_{L=0}^{2} \prod_{1 \leq i<j \leq 2 N_{f}} \Gamma\left(U^{J+\frac{K L}{2}} s_{i} s_{j} ; p, q\right) \prod_{J=0}^{\frac{K-3}{2}} \prod_{j=1}^{2 N_{f}} \Gamma\left(U^{2 J+1+\frac{K}{2}} s_{j}^{2} ; p, q\right) \\
& \times \int_{\mathbb{T}^{\widetilde{N}}} \prod_{1 \leq i<j \leq \widetilde{N}} \frac{\Gamma\left(U z_{i}^{ \pm 1} z_{j}^{ \pm 1}, U^{\frac{K}{2}} z_{i}^{ \pm 1} z_{j}^{ \pm 1} ; p, q\right)}{\Gamma\left(z_{i}^{ \pm 1} z_{j}^{ \pm 1} ; p, q\right)} \prod_{i=1}^{2 N_{f}} \prod_{j=1}^{\widetilde{N}} \frac{\Gamma\left(U^{1-\frac{K}{2}} s_{i}^{-1} z_{j}^{ \pm 1} ; p, q\right)}{\Gamma\left(z_{j}^{ \pm 2} ; p, q\right)} \prod_{j=1}^{\widetilde{N}} \frac{d z_{j}}{2 \pi \mathrm{i} z_{j}}
\end{aligned}
$$

10.4. Symmetric and anti-symmetric tensor matter fields. This duality was found in [8]. The electric theory:

\begin{tabular}{|c|c|c|c|}
\hline & $S P(2 N)$ & $S U\left(2 N_{f}\right)$ & $U(1)_{R}$ \\
\hline$Q$ & $f$ & $f$ & $1-\frac{N+2 K-1}{(K+1) N_{f}}$ \\
$X$ & $T_{A}$ & 1 & $\frac{2}{K+1}$ \\
$Y$ & $T_{S}$ & 1 & $\frac{K}{K+1}$ \\
\hline
\end{tabular}

The magnetic theory:

\begin{tabular}{|c|c|c|c|}
\hline & $S P(2 \widetilde{N})$ & $S U\left(2 N_{f}\right)$ & $U(1)_{R}$ \\
\hline$q$ & $f$ & $\bar{f}$ & $1-\frac{N+2 K-1}{(K+1) N_{f}}$ \\
$\widetilde{X}$ & $T_{A}$ & 1 & $\frac{2}{K+1}$ \\
$\widetilde{Y}$ & $T_{S}$ & 1 & $\frac{K}{K+1}$ \\
$M_{J 0}, J=0, \ldots, K-1$ & 1 & $T_{A}$ & $2-\frac{N+2 K+1}{(K+1) N_{f}}+\frac{2 J}{K+1}$ \\
$M_{2 J 1}, J=0, \ldots, \frac{K-1}{2}$ & 1 & $T_{S}$ & $2-\frac{N+2 K+1}{(K+1) N_{f}}+\frac{2(2 J)}{K+1}+\frac{K}{K+1}$ \\
$M_{2 J+1}, J=0, \ldots, \frac{K-3}{2}$ & 1 & $T_{A}$ & $2-\frac{N+2 K+1}{(K+1) N_{f}}+\frac{2(2 J+1)}{K+1}+\frac{K}{K+1}$ \\
$M_{J 2}, J=0, \ldots, K-1$ & 1 & $T_{A}$ & $2-\frac{N+2 K+1}{(K+1) N_{f}}+\frac{2 J}{K+1}+\frac{2 K}{K+1}$ \\
\hline
\end{tabular}

Here $K$ is odd and

$$
\widetilde{N}=3 K N_{f}-4 K+2-N
$$

For these theories we have the following superconformal indices

$$
\begin{aligned}
I_{E}= & \frac{(p ; p)_{\infty}^{N}(q ; q)_{\infty}^{N}}{2^{N} N !} \Gamma(U ; p, q)^{N-1} \Gamma\left(U^{\frac{K}{2}} ; p, q\right)^{N} \\
\times & \int_{\mathbb{T}^{N}} \prod_{1 \leq i<j \leq N} \frac{\Gamma\left(U z_{i}^{ \pm 1} z_{j}^{ \pm 1}, U^{\frac{K}{2}} z_{i}^{ \pm 1} z_{j}^{ \pm 1} ; p, q\right)}{\Gamma\left(z_{i}^{ \pm 1} z_{j}^{ \pm 1} ; p, q\right)} \prod_{j=1}^{N} \frac{\Gamma\left(U^{\frac{K}{2}} z_{j}^{ \pm 2} ; p, q\right) \prod_{i=1}^{2 N_{f}} \Gamma\left(s_{i} z_{j}^{ \pm 1} ; p, q\right)}{\Gamma\left(z_{j}^{ \pm 2} ; p, q\right)} \prod_{j=1}^{N} \frac{d z_{j}}{2 \pi \mathrm{i} z_{j}}
\end{aligned}
$$


where $U=(p q)^{\frac{1}{K+1}}$ and the balancing condition reads $U^{N+2 K-1} \prod_{i=1}^{2 N_{f}} s_{i}=(p q)^{N_{f}}$, and

$$
\begin{aligned}
I_{M}= & \frac{(p ; p)_{\infty}^{\widetilde{N}}(q ; q)_{\infty}^{\widetilde{N}}}{2^{\widetilde{N}} \widetilde{N} !} \Gamma(U ; p, q)^{\widetilde{N}-1} \Gamma\left(U^{\frac{K}{2}} ; p, q\right)^{\widetilde{N}} \prod_{J=0}^{K-1} \prod_{L=0}^{2} \prod_{1 \leq i<j \leq 2 N_{f}} \Gamma\left(U^{J+\frac{K L}{2}} s_{i} s_{j} ; p, q\right) \\
& \times \prod_{J=0}^{\frac{K-1}{2}} \prod_{j=1}^{2 N_{f}} \Gamma\left(U^{2 J+\frac{K}{2}} s_{j}^{2} ; p, q\right) \int_{\mathbb{T}^{\widetilde{N}}} \prod_{1 \leq i<j \leq \widetilde{N}} \frac{\Gamma\left(U z_{i}^{ \pm 1} z_{j}^{ \pm 1}, U^{\frac{K}{2}} z_{i}^{ \pm 1} z_{j}^{ \pm 1} ; p, q\right)}{\Gamma\left(z_{i}^{ \pm 1} z_{j}^{ \pm 1} ; p, q\right)} \\
& \times \prod_{j=1}^{\widetilde{N}} \frac{\Gamma\left(U^{\frac{K}{2}} z_{j}^{ \pm 2} ; p, q\right) \prod_{i=1}^{2 N_{f}} \Gamma\left(U^{1-\frac{K}{2}} s_{i}^{-1} z_{j}^{ \pm 1} ; p, q\right)}{\Gamma\left(z_{j}^{ \pm 2} ; p, q\right)} \prod_{j=1}^{\widetilde{N}} \frac{d z_{j}}{2 \pi \mathrm{i} z_{j}} .
\end{aligned}
$$

The equalities $I_{E}=I_{M}$ for all the dualities described in this section represent new elliptic hypergeometric identities requiring a rigorous mathematical confirmation.

\section{Some OTHER NEW DUALities}

Let us denote

$$
I_{A_{N}}(\underline{t}, \underline{u} ; p, q)=\frac{(p ; p)_{\infty}^{N}(q ; q)_{\infty}^{N}}{(N+1) !} \int_{\mathbb{T}^{N}} \frac{\prod_{i=1}^{N+1} \prod_{r=1}^{N+3} \Gamma\left(t_{r} z_{i}, u_{r} z_{i}^{-1} ; p, q\right)}{\prod_{1 \leq i<j \leq N+1} \Gamma\left(z_{i} z_{j}^{-1}, z_{i}^{-1} z_{j} ; p, q\right)} \prod_{j=1}^{N} \frac{d z_{j}}{2 \pi \mathrm{i} z_{j}}
$$

with $\prod_{j=1}^{N+1} z_{j}=1$ and the balancing condition $\prod_{i=1}^{N+3} t_{i} u_{i}=(p q)^{2}$, and

$$
I_{B C_{N}}(\underline{t} ; p, q)=\frac{(p ; p)_{\infty}^{N}(q ; q)_{\infty}^{N}}{2^{N} N !} \int_{\mathbb{T}^{N}} \frac{\prod_{i=1}^{N} \prod_{r=1}^{2 N+6} \Gamma\left(t_{r} z_{i}^{ \pm 1} ; p, q\right)}{\prod_{1 \leq i<j \leq N} \Gamma\left(z_{i}^{ \pm 1} z_{j}^{ \pm 1} ; p, q\right) \prod_{j=1}^{N} \Gamma\left(z_{j}^{ \pm 2} ; p, q\right)} \prod_{j=1}^{N} \frac{d z_{j}}{2 \pi \mathrm{i} z_{j}}
$$

with the balancing condition $\prod_{r=1}^{2 N+6} t_{r}=(p q)^{2}$.

\begin{tabular}{|c|c|c|c|c|c|c|c|c|}
\hline & \multicolumn{2}{|c|}{$S U(N+1)$} & \multicolumn{2}{|c|}{$S U(N+3)$} & \multicolumn{2}{|c|}{$S U(N+3)$} & $\overline{U(1)_{B}}$ & $U(1)_{R}$ \\
\hline$\overline{Q_{1}}$ & & \multicolumn{2}{|c|}{$f$} & \multicolumn{2}{|c|}{1} & 2 & $\frac{2}{N+3}$ \\
\hline$Q_{2}$ & \multicolumn{2}{|l|}{$\frac{f}{f}$} & \multicolumn{2}{|l|}{1} & \multicolumn{2}{|l|}{$f$} & -2 & $\frac{2}{N+3}$ \\
\hline & $S P(2 N)$ & $S L$ & $(N+3)$ & & $(N+3)$ & & $J(1)_{B}$ & $U(1)_{R}$ \\
\hline$q_{1}$ & $f$ & & $f$ & & 1 & & $N+1)$ & $\frac{2}{N+3}$ \\
\hline$q_{2}$ & $\bar{f}$ & & 1 & & $f$ & & $N+1$ & $\frac{2}{N+3}$ \\
\hline$X_{1}$ & 1 & & $\bar{T}_{A}$ & & 1 & & $N+1)$ & $2 \frac{N+1}{N+3}$ \\
\hline$X_{2}$ & 1 & & 1 & & $\bar{T}_{A}$ & & $(N+1)$ & $2 \frac{N+1}{N+3}$ \\
\hline
\end{tabular}

11.1. $S U \leftrightarrow S P$ groups mixing duality. The first case electric gauge group is $G=S U(N+$ 1 ), but the dual gauge group is of a different type $G=S P(2 N)$. The flavor symmetry group in both cases is $F=S U(N+3) \times S U(N+3) \times U(1)_{B}$. The field content of dual theories is described in the tables below

The superconformal indices are

$$
\begin{aligned}
& I_{E}=I_{A_{N}}\left(t_{1}, \ldots, t_{N+3}, u_{1}, \ldots, u_{N+3} ; p, q\right), \\
& I_{M}=\prod_{1 \leq i<j \leq N+3} \Gamma\left(T / t_{i} t_{j}, U / u_{i} u_{j} ; p, q\right) I_{B C_{N}}\left(\ldots(U / T)^{1 / 4} t_{i} \ldots, \ldots(T / U)^{1 / 4} u_{i} \ldots ; p, q\right),
\end{aligned}
$$

where $T=\prod_{1 \leq i \leq N+3} t_{i}$ and $U=\prod_{1 \leq i \leq N+3} u_{i}$.

The equality $\bar{I}_{E}=I_{M}$ represents the mixed elliptic hypergeometric integrals transformation proven in [65]. We used this identity as a starting point for finding the described new Seibergtype pair of field theories. 
11.2. $S U \leftrightarrow S U$ groups mixing duality. Again, we use consequences of the mixed transformations derived in [65]. Corresponding dualities have the flavor symmetry groups

$$
F=S U(K) \times S U(N+2-K) \times U(1)_{1} \times S U(K) \times S U(N+2-K) \times U(1)_{2} \times U(1)_{B},
$$

for arbitrary $0<K<N+2$. The matter field content of the initial electric field theory is given in the table

\begin{tabular}{|c|c|c|c|c|c|}
\hline & $S U(N)$ & $S U(N+2)$ & $S U(N+2)$ & $U(1)_{B}$ & $U(1)_{R}$ \\
\hline$Q_{1}$ & $f$ & $f$ & 1 & 1 & $\frac{2}{N+2}$ \\
$Q_{2}$ & $\bar{f}$ & 1 & $f$ & -1 & $\frac{2}{N+2}$ \\
\hline
\end{tabular}

In order to verify the 't Hooft anomalies matching conditions for relevant flavor symmetry subgroups, it is useful to rewrite the latter table as

\begin{tabular}{|c|c|c|c|c|c|c|c|c|c|}
\hline & $S U(N)$ & $S U(K)$ & $S U(M)$ & $U(1)_{1}$ & $S U(K)$ & $S U(M)$ & $U(1)_{2}$ & $U(1)_{B}$ & $U(1)_{R}$ \\
\hline$q_{1}$ & $f$ & $f$ & 1 & $M$ & 1 & 1 & 0 & 1 & $\frac{2}{N+2}$ \\
$q_{2}$ & $f$ & 1 & $f$ & $-K$ & 1 & 1 & 0 & 1 & $\frac{2}{N+2}$ \\
$q_{3}$ & $\bar{f}$ & 1 & 1 & 0 & $f$ & 1 & $M$ & -1 & $\frac{2}{N+2}$ \\
$q_{4}$ & $\bar{f}$ & 1 & 1 & 0 & 1 & $f$ & $-K$ & -1 & $\frac{2}{N+2}$ \\
\hline
\end{tabular}

where $M=N+2-K$. The dual theory content is described in the following table

\begin{tabular}{|c|c|c|c|c|c|c|c|c|c|}
\hline & $S U(N)$ & $S U(K)$ & $S U(M)$ & $U(1)_{1}$ & $S U(K)$ & $S U(M)$ & $U(1)_{2}$ & $U(1)_{B}$ & $U(1)_{R}$ \\
\hline$q_{1}$ & $\bar{f}$ & $f$ & 1 & $\frac{K(K-2)}{N}-K+M$ & 1 & 1 & $\frac{M K}{N}$ & $1-M$ & $\frac{2}{N+2}$ \\
$q_{2}$ & $f$ & 1 & $f$ & $-\frac{K(K-2)}{N}$ & 1 & 1 & $\frac{-M K}{N}$ & $1-K$ & $\frac{2}{N+2}$ \\
$q_{3}$ & $f$ & 1 & 1 & $\frac{M K}{N}$ & $f$ & 1 & $\frac{K(K-2)}{N}-K+M$ & $M-1$ & $\frac{2}{N+2}$ \\
$q_{4}$ & $\bar{f}$ & 1 & 1 & $\frac{-M K}{N}$ & 1 & $f$ & $-\frac{K(K-2)}{N}$ & $K-1$ & $\frac{2}{N+2}$ \\
$X_{1}$ & 1 & $f$ & 1 & $M$ & 1 & $f$ & $-K$ & 0 & $\frac{4}{N+2}$ \\
$X_{2}$ & 1 & 1 & $f$ & $-K$ & $f$ & 1 & $M$ & 0 & $\frac{4}{N+2}$ \\
$Y_{1}$ & 1 & $\bar{f}$ & $\bar{f}$ & $K-M$ & 1 & 1 & 0 & $N$ & $\frac{2 N}{N+2}$ \\
$Y_{2}$ & 1 & 1 & 1 & 0 & $\bar{f}$ & $\bar{f}$ & $K-M$ & $-N$ & $\frac{2 N}{N+2}$ \\
\hline
\end{tabular}

The superconformal indices have the form

$$
\begin{aligned}
& I_{E}=I_{A_{N-1}}\left(t_{1}, \ldots, t_{N+2}, u_{1}, \ldots, u_{N+2} ; p, q\right), \\
& I_{M}=\prod_{1 \leq r<K, K \leq s \leq N+2} \Gamma\left(t_{r} u_{s}, t_{s} u_{r}, T / t_{s} t_{r}, U / u_{r} u_{s}\right) I_{A_{N-1}}\left(t_{1}^{\prime}, \ldots, t_{N+2}^{\prime}, u_{1}^{\prime}, \ldots, u_{N+2}^{\prime} ; p, q\right),
\end{aligned}
$$

where $T=\prod_{r=1}^{N+2} t_{r}, U=\prod_{r=1}^{N+2} u_{r}, T_{K}=\prod_{r=1}^{K} t_{r}, U_{K}=\prod_{r=1}^{K} u_{r}$, and

$$
\begin{gathered}
t_{r}^{\prime}=(T / U)^{\frac{N-K}{2 N}}\left(T_{K} / U_{K}\right)^{1 / N} u_{r}, \quad 1 \leq r<K+1, \\
t_{r}^{\prime}=(U / T)^{\frac{K}{2 N}}\left(T_{K} / U_{K}\right)^{1 / N} t_{r}, \quad K+1 \leq r \leq N+2, \\
u_{r}^{\prime}=(U / T)^{\frac{N-K}{2 N}}\left(U_{K} / T_{K}\right)^{1 / N} t_{r}, \quad 1 \leq r<K+1, \\
u_{r}^{\prime}=(T / U)^{\frac{K}{2 N}}\left(U_{K} / T_{K}\right)^{1 / N} u_{r}, \quad K+1 \leq r \leq N+2 .
\end{gathered}
$$

The equality $I_{E}=I_{M}$ for $K=1$ was suggested in [83] and the general relation with the complete proof for arbitrary $K$ is given in [65].

\section{S-CONFINEMENT}

Following $[15,16,76]$, by $s$-confinement we mean smooth confinement without chiral symmetry breaking and with a non-vanishing confining superpotential. The theory is confined when its infrared physics can be described completely in terms of gauge invariant composite fields and their interactions. This description has to be valid everywhere in the moduli space of vacua. $s$-confinement requires also that the theory dynamically generates a confining superpotential. 
Furthermore, the phase without chiral symmetry breaking implies that the origin of the classical moduli space serves also as a vacuum in the quantum theory. In this vacuum all the global symmetries present in the ultraviolet regime remain unbroken. Finally, the confining superpotential is a holomorphic function of the confined degrees of freedom and couplings, which describe all interactions in the extreme infrared. From the point of view of elliptic hypergeometric functions the $s$-confinement means that the dual theory gauge group is trivial $G=1$ (i.e., there is no vector superfield $\widetilde{V}$ ) and the integrals describing superconformal indices are computable exactly, defining highly non-trivial elliptic beta integrals [81].

12.1. $S U(N)$ gauge group. In this section we present known examples of the confining theories with the unitary gauge group. For brevity we combine the electric and magnetic theories in a single table separating them by the double line. The magnetic theory fields are denoted using the conventions of [15].

12.1.1. $S U(N)$ with $(N+1)(f+\bar{f})$. [76]

\begin{tabular}{|c|c|c|c|c|c|}
\hline & $S U(N)$ & $S U(N+1)$ & $S U(N+1)$ & $U(1)$ & $U(1)_{R}$ \\
\hline$Q$ & $f$ & $f$ & 1 & 1 & $\frac{1}{N+1}$ \\
$\widetilde{Q}$ & $\bar{f}$ & 1 & $f$ & -1 & $\frac{1}{N+1}$ \\
\hline \hline$Q \widetilde{Q}$ & & $f$ & $f$ & 0 & $\frac{2}{N+1}$ \\
$Q^{N}$ & & $\bar{f}$ & 1 & $N$ & $\frac{N}{N+1}$ \\
$\widetilde{Q}^{N}$ & & 1 & $\bar{f}$ & $-N$ & $\frac{N}{N+1}$ \\
\hline
\end{tabular}

The superconformal indices for these theories are equal to (after appropriate renormalization of the parameters)

$$
\begin{gathered}
I_{E}=\frac{(p ; p)_{\infty}^{N-1}(q ; q)_{\infty}^{N-1}}{N !} \int_{\mathbb{T}^{N-1}} \prod_{1 \leq j<k \leq N} \frac{1}{\Gamma\left(z_{i} z_{j}^{-1}, z_{i}^{-1} z_{j} ; p, q\right)} \\
\times \prod_{j=1}^{N} \prod_{m=1}^{N+1} \Gamma\left(s_{m} z_{j}, t_{m} z_{j}^{-1} ; p, q\right) \prod_{j=1}^{N-1} \frac{d z_{j}}{2 \pi \mathrm{i} z_{j}},
\end{gathered}
$$

where $\prod_{j=1}^{N} z_{j}=1$, and

$$
I_{M}=\prod_{m=1}^{N+1} \Gamma\left(S s_{m}^{-1}, T t_{m}^{-1} ; p, q\right) \prod_{k, m=1}^{N+1} \Gamma\left(s_{k} t_{m} ; p, q\right)
$$

where $S=\prod_{m=1}^{N+1} s_{m}, T=\prod_{m=1}^{N+1} t_{m}$, with the balancing condition $S T=p q$.

The exact evaluation formula for the integral $I_{E}=I_{M}$ was conjectured and partially confirmed in [83]. Its complete proofs are given in $[65,86]$. In the simplest $p \rightarrow 0$ limit it is reduced to one of the Gustafson integrals [35].

12.1.2. $S U(2 N)$ with $T_{A}+2 N \bar{f}+4 f$. The theory with $G=S U(2 N)$ gauge group and flavor group $F=S U(2 N) \times S U(4) \times U(1)_{1} \times U(1)_{2}$ was found to be confining in [62, 64]. The field content of both theories is described in the table below 


\begin{tabular}{|c|c|c|c|c|c|c|}
\hline & $S U(2 N)$ & $S U(2 N)$ & $S U(4)$ & $U(1)_{1}$ & $U(1)_{2}$ & $U(1)_{R}$ \\
\hline$Q$ & $f$ & 1 & $f$ & $-2 N$ & $-2 N+2$ & $\frac{1}{2}$ \\
$\widetilde{Q}$ & $\bar{f}$ & $f$ & 1 & 4 & $-2 N+2$ & 0 \\
$A$ & $T_{A}$ & 1 & 1 & 0 & $2 N+4$ & 0 \\
\hline \hline$Q \widetilde{Q}$ & & $f$ & $f$ & $4-2 N$ & $-4 N+4$ & $\frac{1}{2}$ \\
$A \widetilde{Q}^{2}$ & & $T_{A}$ & 1 & 8 & $-2 N+8$ & 0 \\
$A^{N}$ & & 1 & 1 & 0 & $2 N^{2}+4 N$ & 0 \\
$A^{N-1} Q^{2}$ & & 1 & $T_{A}$ & $-4 N$ & $2 N^{2}-2 N$ & 1 \\
$A^{N-1} Q^{4}$ & & 1 & 1 & $-8 N$ & $2 N^{2}-8 N$ & 2 \\
$\widetilde{Q}^{2 N}$ & & 1 & 1 & $8 N$ & $-4 N^{2}+4 N$ & 0 \\
\hline
\end{tabular}

We come to the following integrals describing the superconformal indices

$$
\begin{gathered}
I_{E}=\frac{(p ; p)_{\infty}^{2 N-1}(q ; q)_{\infty}^{2 N-1}}{(2 N) !} \int_{\mathbb{T}^{2 N-1}} \prod_{1 \leq j<k \leq 2 N} \frac{\Gamma\left(t z_{i} z_{j} ; p, q\right)}{\Gamma\left(z_{i} z_{j}^{-1}, z_{i}^{-1} z_{j} ; p, q\right)} \\
\times \prod_{j=1}^{2 N} \prod_{k=1}^{2 N} \Gamma\left(t_{k} z_{j}^{-1} ; p, q\right) \prod_{i=1}^{4} \Gamma\left(s_{i} z_{j} ; p, q\right) \prod_{j=1}^{2 N-1} \frac{d z_{j}}{2 \pi \mathrm{i} z_{j}},
\end{gathered}
$$

with $\prod_{j=1}^{2 N} z_{j}=1$, and

$$
I_{M}=\prod_{1 \leq j<k \leq 2 N} \Gamma\left(t t_{j} t_{k} ; p, q\right) \prod_{k=1}^{2 N} \prod_{i=1}^{4} \Gamma\left(t_{k} s_{i} ; p, q\right) \frac{\Gamma\left(t^{N}, T ; p, q\right)}{\Gamma\left(t^{N} T ; p, q\right)} \prod_{1 \leq i<m \leq 4} \Gamma\left(t^{\frac{2 N-2}{2}} s_{i} s_{m} ; p, q\right),
$$

with the balancing condition $t^{2 N-2} S T=p q$, where $S=\prod_{i=1}^{4} s_{i}, T=\prod_{j=1}^{2 N} t_{j}$.

Equality $I_{E}=I_{M}$ defines the elliptic beta integral introduced in [83]. It represents an elliptic extension of the Gustafson-Rakha $q$-beta integral for odd number of integration variables [38].

12.1.3. $S U(2 N+1)$ with $T_{A}+(2 N+1) \bar{f}+4 f$. These dual models were considered in [62, 64]:

\begin{tabular}{|c|c|c|c|c|c|c|}
\hline & $S U(2 N+1)$ & $S U(2 N+1)$ & $S U(4)$ & $U(1)_{1}$ & $U(1)_{2}$ & $U(1)_{R}$ \\
\hline$Q$ & $f$ & 1 & $f$ & $-2 N-1$ & $-2 N+1$ & $\frac{1}{2}$ \\
$\widetilde{Q}$ & $\bar{f}$ & $f$ & 1 & 4 & $-2 N+1$ & 0 \\
$A$ & $T_{A}$ & 1 & 1 & 0 & $2 N+5$ & 0 \\
\hline \hline$Q \widetilde{Q}$ & & $f$ & $f$ & $3-2 N$ & $-4 N+2$ & $\frac{1}{2}$ \\
$A \widetilde{Q}{ }^{2}$ & & $T_{A}$ & 1 & 8 & $-2 N+7$ & 0 \\
$A^{N} Q$ & & 1 & $f$ & $-2 N-1$ & $2 N^{2}+3 N+1$ & $\frac{1}{2}$ \\
$A^{N-1} Q^{3}$ & & 1 & $\frac{f}{f}$ & $-6 N-3$ & $2 N^{2}-3 N-2$ & $\frac{3}{2}$ \\
$\widetilde{Q}^{2 N+1}$ & & 1 & 1 & $8 N+4$ & $-4 N^{2}+1$ & 0 \\
\hline
\end{tabular}

The indices have the form

$$
\begin{array}{r}
I_{E}=\frac{(p ; p)_{\infty}^{2 N}(q ; q)_{\infty}^{2 N}}{(2 N+1) !} \int_{\mathbb{T}^{2 N}} \prod_{1 \leq j<k \leq 2 N+1} \frac{\Gamma\left(t z_{i} z_{j} ; p, q\right)}{\Gamma\left(z_{i} z_{j}^{-1}, z_{i}^{-1} z_{j} ; p, q\right)} \\
\quad \times \prod_{j=1}^{2 N+1} \prod_{k=1}^{2 N+1} \Gamma\left(t_{k} z_{j}^{-1} ; p, q\right) \prod_{i=1}^{4} \Gamma\left(s_{i} z_{j} ; p, q\right) \prod_{j=1}^{2 N} \frac{d z_{j}}{2 \pi \mathrm{i} z_{j}},
\end{array}
$$


with $\prod_{j=1}^{2 N+1} z_{j}=1$, and

$$
I_{M}=\prod_{1 \leq j<k \leq 2 N+1} \Gamma\left(t t_{j} t_{k} ; p, q\right) \prod_{k=1}^{2 N+1} \prod_{i=1}^{4} \Gamma\left(t_{k} s_{i} ; p, q\right) \Gamma(T ; p, q) \prod_{i=1}^{4} \frac{\Gamma\left(t^{N} s_{i} ; p, q\right)}{\Gamma\left(t^{N} T s_{i} ; p, q\right)}
$$

where the balancing condition reads $t^{2 N-1} S T=p q$ and $T=\prod_{k=1}^{2 N+1} t_{k}, S=\prod_{k=1}^{4} s_{k}$.

The equality $I_{E}=I_{M}$ was also suggested in [83] as an elliptic extension of the GustafsonRakha $q$-beta integral with an even number of integrations [38].

12.1.4. $S U(2 N+1)$ with $T_{A}+\bar{T}_{A}+3 \bar{f}+3 f$. Models [16]:

\begin{tabular}{|c|c|c|c|c|c|c|c|}
\hline & $S U(2 N+1)$ & $S U(3)$ & $S U(3)$ & $U(1)_{1}$ & $U(1)_{2}$ & $U(1)_{B}$ & $U(1)_{R}$ \\
\hline$Q$ & $f$ & $f$ & 1 & 0 & $2 N-1$ & 1 & $\frac{1}{3}$ \\
$\widetilde{Q}$ & $\bar{f}$ & 1 & $f$ & 0 & $2 N-1$ & -1 & $\frac{1}{3}$ \\
$A$ & $T_{A}$ & 1 & 1 & 1 & -3 & 0 & 0 \\
$\widetilde{A}$ & $\bar{T}_{A}$ & 1 & 1 & -1 & -3 & 0 & 0 \\
\hline \hline$Q(A \widetilde{A})^{k} \widetilde{Q}$ & & $f$ & $f$ & 0 & $4 N-2-6 k$ & 0 & $\frac{2}{3}$ \\
$\widetilde{A}(A \widetilde{A})^{k} Q^{2}$ & & $T_{A}$ & 1 & -1 & $4 N-5-6 k$ & 2 & $\frac{2}{3}$ \\
$A(A \widetilde{A})^{k} \widetilde{Q}^{2}$ & & 1 & $T_{A}$ & 1 & $4 N-5-6 k$ & -2 & $\frac{2}{3}$ \\
$A^{N} Q$ & & $f$ & 1 & $N$ & $-N-1$ & 1 & $\frac{1}{3}$ \\
$\widetilde{A}^{N} \widetilde{Q}$ & & 1 & $f$ & $-N$ & $-N-1$ & -1 & $\frac{1}{3}$ \\
$A^{N-1} Q^{3}$ & & 1 & 1 & $N-1$ & $3 N$ & 3 & 1 \\
$\widetilde{A}^{N-1} \widetilde{Q}^{3}$ & & 1 & 1 & $-N+1$ & $3 N$ & -3 & 1 \\
$(A \widetilde{A})^{m}$ & & 1 & 1 & 0 & $-6 m$ & 0 & 0 \\
\hline
\end{tabular}

where $k=0, \ldots, N-1$ and $m=1, \ldots, N$.

The superconformal indices are written as

$$
\begin{aligned}
I_{E}=\frac{(p ; p)_{\infty}^{2 N}(q ; q)_{\infty}^{2 N}}{(2 N+1) !} \int_{\mathbb{T}^{2 N}} \prod_{1 \leq i<j \leq 2 N+1} \frac{\Gamma\left(U z_{i} z_{j}, V z_{i}^{-1} z_{j}^{-1} ; p, q\right)}{\Gamma\left(z_{i} z_{j}^{-1}, z_{i}^{-1} z_{j} ; p, q\right)} \\
\quad \times \prod_{i=1}^{3} \prod_{j=1}^{2 N+1} \Gamma\left(s_{i} z_{j}, t_{i} z_{j}^{-1} ; p, q\right) \prod_{j=1}^{2 N} \frac{d z_{j}}{2 \pi \mathrm{i} z_{j}},
\end{aligned}
$$

where $\prod_{j=1}^{2 N+1} z_{j}=1$, and

$$
\begin{aligned}
& I_{M}=\prod_{i=1}^{3} \Gamma\left(U^{N} s_{i}, V^{N} t_{i} ; p, q\right) \Gamma\left(U^{N-1} s_{1} s_{2} s_{3}, V^{N-1} t_{1} t_{2} t_{3} ; p, q\right) \prod_{j=1}^{N} \Gamma\left((U V)^{j} ; p, q\right) \\
& \times \prod_{j=0}^{N-1}\left[\prod_{i, k=1}^{3} \Gamma\left((U V)^{j} s_{i} t_{k} ; p, q\right) \prod_{1 \leq i<k \leq 3} \Gamma\left(V(U V)^{j} s_{i} s_{k}, U(U V)^{j} t_{i} t_{k} ; p, q\right)\right],
\end{aligned}
$$

where the balancing condition reads $(U V)^{2 N-1} \prod_{i=1}^{3} s_{i} t_{i}=p q$.

The equality $I_{E}=I_{M}$ was derived by Spiridonov in [83] by purely algebraic means as a consequence of other elliptic beta integrals. In the simplest $p \rightarrow 0$ limit it reduces to Gustafson's $q$-beta integral for the root system $A_{2 N}[37]$. 
12.1.5. $S U(2 N)$ with $T_{A}+\bar{T}_{A}+3 \bar{f}+3 f$. For $N>2$ the models have the form [16]:

\begin{tabular}{|c|c|c|c|c|c|c|c|}
\hline & $S U(2 N)$ & $S U(3)$ & $S U(3)$ & $U(1)_{1}$ & $U(1)_{2}$ & $U(1)_{B}$ & $U(1)_{R}$ \\
\hline$Q$ & $f$ & $f$ & 1 & 0 & $2 N-2$ & 1 & $\frac{1}{3}$ \\
$\widetilde{Q}$ & $\bar{f}$ & 1 & $f$ & 0 & $2 N-2$ & -1 & $\frac{1}{3}$ \\
$A$ & $T_{A}$ & 1 & 1 & 1 & -3 & 0 & 0 \\
$\widetilde{A}$ & $\bar{T}_{A}$ & 1 & 1 & -1 & -3 & 0 & 0 \\
\hline \hline$Q(A \widetilde{A})^{k} \widetilde{Q}$ & & $f$ & $f$ & 0 & $4 N-4-6 k$ & 0 & $\frac{2}{3}$ \\
$\widetilde{A}(A \widetilde{A})^{m} Q^{2}$ & & $T_{A}$ & 1 & -1 & $4 N-7-6 m$ & 2 & $\frac{2}{3}$ \\
$A(A \widetilde{A})^{m} \widetilde{Q}^{2}$ & & 1 & $T_{A}$ & 1 & $4 N-7-6 m$ & -2 & $\frac{2}{3}$ \\
$A^{N}$ & & 1 & 1 & $N$ & $-3 N$ & 0 & 0 \\
$\widetilde{A}^{N}$ & & 1 & 1 & $-N$ & $-3 N$ & 0 & 0 \\
$A^{N-1} Q^{2}$ & & $T_{A}$ & 1 & $N-1$ & $N-1$ & 2 & $\frac{2}{3}$ \\
$\widetilde{A}^{N-1} \widetilde{Q}^{2}$ & & 1 & $T_{A}$ & $-N+1$ & $N-1$ & -2 & $\frac{2}{3}$ \\
$(A \widetilde{A})^{n}$ & & 1 & 1 & 0 & $-6 n$ & 0 & 0 \\
\hline
\end{tabular}

where $k=0, \ldots, N-1, m=0, \ldots, N-2$ and $n=1, \ldots, N-1$. For $N=2$ the flavor group is enlarged to $F=S U(3) \times S U(3) \times S U(2) \times U(1)_{2} \times U(1)_{B}$, and the fields $A, \tilde{A}$ unify to the $S U(2)$-group doublet.

The expressions for the superconformal indices are

$$
\begin{aligned}
I_{E}=\frac{(p ; p)_{\infty}^{2 N-1}(q ; q)_{\infty}^{2 N-1}}{(2 N) !} \int_{\mathbb{T}^{2 N-1}} \prod_{1 \leq i<j \leq 2 N} \frac{\Gamma\left(U z_{i} z_{j}, V z_{i}^{-1} z_{j}^{-1} ; p, q\right)}{\Gamma\left(z_{i} z_{j}^{-1}, z_{i}^{-1} z_{j} ; p, q\right)} \\
\times \prod_{j=1}^{2 N} \prod_{i=1}^{3} \Gamma\left(s_{i} z_{j}, t_{i} z_{j}^{-1} ; p, q\right) \prod_{j=1}^{2 N-1} \frac{d z_{j}}{2 \pi \mathrm{i} z_{j}},
\end{aligned}
$$

with $\prod_{j=1}^{2 N} z_{j}=1$, and

$$
\begin{aligned}
I_{M}= & \Gamma\left(U^{N}, V^{N} ; p, q\right) \prod_{1 \leq i<k \leq 3} \Gamma\left(U^{N-1} s_{i} s_{k}, V^{N-1} t_{i} t_{k} ; p, q\right) \prod_{j=1}^{N} \prod_{i, k=1}^{3} \Gamma\left((U V)^{j-1} s_{i} t_{k} ; p, q\right) \\
& \times \prod_{j=1}^{N-1} \Gamma\left((U V)^{j} ; p, q\right) \prod_{j=0}^{N-2} \prod_{1 \leq i<k \leq 3} \Gamma\left(V(U V)^{j} s_{i} s_{k}, U(U V)^{j} t_{i} t_{k} ; p, q\right),
\end{aligned}
$$

where the balancing condition reads $(U V)^{2 N-2} \prod_{i=1}^{3} s_{i} t_{i}=p q$. The equality $I_{E}=I_{M}$ was also derived in [83] as a consequence of some other elliptic beta integrals. In the simplest $p \rightarrow 0$ limit, it reduces to one of Gustafson's integrals for the root system $A_{2 N-1}$ [37]. Similar to the case of non-confining $N_{f}=4$ dualities described earlier, a careful examination of the limit $V \rightarrow 1$ (or $U \rightarrow 1$ ) shows that the equality of superconformal indices in this case reduces to the equality of $S P(2 N)$-group confining duality indices discussed in [91]. This means that the elliptic Selberg integral introduced in [19] (see integral (12.35) and its evaluation (12.36) below) is a limiting case of Spiridonov's $A_{n}$-elliptic beta integral. This result could have been expected since the computation of the latter integral in [83] used the elliptic Selberg integral.

12.1.6. $S U\left(K N_{f}-1\right)$ with $N_{f} f+N_{f} \bar{f}+1 a d j$. Taking $N=K N_{f}-1$ in (9.1) (or, $\left.\widetilde{N}=1\right)$, we find the $s$-confining dual theory discussed in [14]. The field content of these theories is easily found from the tables given in Sect. 9.1. Namely, in the electric theory one should fix $N$ as 
described; on the magnetic side one should keep all the mesons and baryons and set $\widetilde{N}=1$ in the gauge group part. Therefore for this case the superconformal index for the electric theory is given by (9.2), and the magnetic superconformal index takes the form

$$
I_{M}=\prod_{l=1}^{K} \prod_{1 \leq i, j \leq N_{f}} \Gamma\left(U^{l-1} s_{i} t_{j}^{-1} ; p, q\right) \prod_{i=1}^{N_{f}} \Gamma\left(U(S T)^{\frac{K}{2}} s_{i}^{-1}, U(S T)^{-\frac{K}{2}} t_{i} ; p, q\right)
$$

where $U=(p q)^{\frac{1}{K+1}}, S=\prod_{j=1}^{N_{f}} s_{j}, T=\prod_{j=1}^{N_{f}} t_{j}$, and the balancing condition reads $U^{2 K N_{f}-2} S T^{-1}=$ $(p q)^{N_{f}}$.

For $K=1$ one obtains the known $A_{N}$-root systems integral of type I from Sect. 12.1.1. The conjecture $I_{E}=I_{M}$ for $K>1$ represents a new elliptic beta integral requiring rigorous mathematical justification.

12.1.7. $S U\left(3 K N_{f}-1\right)$ with $N_{f} f+N_{f} \bar{f}+2 a d j$. If we set $N=3 K N_{f}-1$ in (9.4), then we obtain the $s$-confinement discussed in [50]. The superconformal index for the electric theory is given by (9.5), and the magnetic superconformal index takes the form

$$
\begin{aligned}
I_{M}= & \prod_{L=0}^{K-1} \prod_{J=0}^{2} \Gamma\left(U^{L+K J / 2} s_{i} t_{j}^{-1} ; p, q\right) \\
& \times \prod_{i=1}^{N_{f}} \Gamma\left(U^{\frac{2-K}{2}}(S T)^{\frac{3 K}{2 N}} s_{i}^{-1}, U^{\frac{2-K}{2}}(S T)^{-\frac{3 K}{2 N}} t_{i} ; p, q\right),
\end{aligned}
$$

where $U=(p q)^{\frac{1}{K+1}}, S=\prod_{j=1}^{N_{f}} s_{j}, T=\prod_{j=1}^{N_{f}} t_{j}$, and the balancing condition reads $U^{N} S T^{-1}=$ $(p q)^{N_{f}}$. The equality $I_{E}=I_{M}$ is a new conjectural elliptic beta integral.

12.1.8. $S U\left((2 K+1) N_{f}-4 K-1\right)$ with $N_{f} f+N_{f} \bar{f}+2 T_{A}$. If we set $N=(2 K+1) N_{f}-4 K-1$ in (9.7), we obtain the $s$-confinement discussed by Klein in [50]. The electric superconformal index is given by (9.8), and the magnetic superconformal index takes the form

$$
\begin{aligned}
I_{M}= & \prod_{j=0}^{K} \prod_{k, l=1}^{N_{f}} \Gamma\left((p q)^{\frac{j}{K+1}} s_{k} t_{l} ; p, q\right) \prod_{k=1}^{N_{f}} \Gamma\left((U \widetilde{U})^{\frac{1}{2}} s_{k}^{-1},(U \widetilde{U})^{-\frac{1}{2}}(p q)^{\frac{1}{K+1}} t_{k}^{-1} ; p, q\right) \\
& \times \prod_{r=0}^{K-1} \prod_{1 \leq k<l \leq N_{f}} \Gamma\left(U^{-1}(p q)^{\frac{r+1}{K+1}} s_{k} s_{l}, U(p q)^{\frac{r}{K+1}} t_{k} t_{l} ; p, q\right),
\end{aligned}
$$

where $U$ is an arbitrary parameter, $\widetilde{U}=U^{2 K N_{f}-4 K-1} S T^{-1}(p q)^{\frac{1-K N_{f}+2 K}{K+1}}, S=\prod_{j=1}^{N_{f}} s_{j}, T=$ $\prod_{j=1}^{N_{f}} t_{j}$, and the balancing condition reads $S T=(p q)^{N_{f}-\frac{N+2 K}{K+1}}$.

For $K=0$ the parameter $U$ drops out, and one obtains the integral discussed in Sect. 12.1.1. The general $K>0$ conjecture $I_{E}=I_{M}$ represents another new elliptic beta integral.

12.1.9. $S U\left((2 K+1) N_{f}+4 K-1\right)$ with $N_{f} f+N_{f} \bar{f}+2 T_{S}$. If we set $N=(2 K+1) N_{f}+4 K-1$ in (9.9), we obtain again the $s$-confinement [50]. The corresponding electric superconformal 
index is given by (9.10), and the magnetic superconformal index takes the form

$$
\begin{aligned}
I_{M}= & \Gamma\left(\widetilde{U}, \widetilde{U}^{-1}(p q)^{\frac{1}{K+1}} ; p, q\right) \prod_{j=0}^{K} \prod_{k, l=1}^{N_{f}} \Gamma\left((p q)^{\frac{j}{K+1}} s_{k} t_{l} ; p, q\right) \\
& \times \prod_{r=0}^{K-1} \prod_{1 \leq k<l \leq N_{f}} \Gamma\left(U^{-1}(p q)^{\frac{r+1}{K+1}} s_{k} s_{l}, U(p q)^{\frac{r}{K+1}} t_{k} t_{l} ; p, q\right) \\
\times & \prod_{k=1}^{N_{f}}\left[\prod_{r=0}^{K-1} \Gamma\left(U^{-1}(p q)^{\frac{r+1}{K+1}} s_{k}^{2}, U(p q)^{\frac{r}{K+1}} t_{k}^{2} ; p, q\right) \Gamma\left((U \widetilde{U})^{\frac{1}{2}} s_{k}^{-1},(U \widetilde{U})^{-\frac{1}{2}}(p q)^{\frac{1}{K+1}} t_{k}^{-1} ; p, q\right)\right]
\end{aligned}
$$

where $\widetilde{U}=U^{2 K N_{f}+4 K-1} S T^{-1}(p q)^{\frac{1-K N_{f}-2 K}{K+1}}, S=\prod_{j=1}^{N_{f}} s_{j}, T=\prod_{j=1}^{N_{f}} t_{j}$, and the balancing condition reads $S T=(p q)^{N_{f}-\frac{N-2 K}{K+1}}$.

Presently the conjecture $I_{E}=I_{M}$ is confirmed only for $K=0$, which reduces again to the integral of Sect. 12.1.1.

12.1.10. $S U\left((4 K+3)\left(N_{f}-4\right)-1\right)$ with $N_{f} f+\left(N_{f}-8\right) \bar{f}+T_{A}+T_{S}$. If we take $N=(4 K+3)\left(N_{f}-\right.$ 4) -1 in (9.12), we obtain the $s$-confinement [50]. The corresponding electric superconformal index is given by (9.13), and the magnetic superconformal index takes the form

$$
\begin{aligned}
I_{M}= & \prod_{J=0}^{2 K+1} \prod_{i=1}^{N_{f}} \prod_{j=1}^{N_{f}-8} \Gamma\left((p q)^{\frac{J}{2(K+1)}} s_{i} t_{j} ; p, q\right) \\
& \times \prod_{l=0}^{2 K} \prod_{1 \leq i<j \leq N_{f}} \Gamma\left((p q)^{\frac{l+1}{2(K+1)}} U^{-1} s_{i} s_{j} ; p, q\right) \prod_{l=0}^{K} \prod_{i=1}^{N_{f}} \Gamma\left((p q)^{\frac{2 l+1}{2(K+1)}} U^{-1} s_{i}^{2} ; p, q\right) \\
& \times \prod_{m=0}^{2 K} \prod_{1 \leq i<j \leq N_{f}-8} \Gamma\left((p q)^{\frac{m}{2(K+1)}} U t_{i} t_{j} ; p, q\right) \prod_{m=0}^{K-1} \prod_{i=1}^{N_{f}-8} \Gamma\left((p q)^{\frac{2 m+1}{2(K+1)}} U t_{i}^{2} ; p, q\right) \\
& \times \Gamma\left(\widetilde{U}^{-1}(p q)^{\frac{1}{2(K+1)}} ; p, q\right) \prod_{k=1}^{N_{f}} \Gamma\left((U \widetilde{U})^{\frac{1}{2}} s_{k}^{-1} ; p, q\right) \prod_{l=1}^{N_{f}-8} \Gamma\left((U \widetilde{U})^{-\frac{1}{2}}(p q)^{\frac{1}{2(K+1)}} t_{l}^{-1} ; p, q\right),
\end{aligned}
$$

where $\widetilde{U}=\left(S^{2} U^{N-N_{f}}\right)^{\frac{1}{N}}$ and the balancing condition reads

$$
U^{-4} \prod_{j=1}^{N_{f}} s_{j} t_{j}=(p q)^{N_{f}-4-\frac{(4 K+3)\left(N_{f}-4\right)+1}{2(K+1)}}
$$

The equality $I_{E}=I_{M}$ represents another conjectural new elliptic beta integral.

12.1.11. $S U\left(3 K N_{f}+3\right)$ with $N_{f} f+N_{f} \bar{f}+a d j+T_{S}+\bar{T}_{S}$. If we take $N=3 K N_{f}+3$ for $K$-odd in (9.15), we obtain again the $s$-confinement [50]. The corresponding electric superconformal 
index is given by (9.16), and the magnetic superconformal index is

$$
\begin{aligned}
I_{M}=\prod_{L=0}^{K-1} & \prod_{i, j=1}^{N_{f}} \Gamma\left(U^{L+K} s_{i} t_{j}^{-1}, U^{L} s_{i} t_{j}^{-1} ; p, q\right) \Gamma\left(U^{\frac{K}{2}} X^{N-K N_{f}} Y^{N}, U^{\frac{K}{2}}\left(X^{N-K N_{f}} Y^{N}\right)^{-1} ; p, q\right) \\
& \times \prod_{J=0}^{K-1} \prod_{1 \leq i<j \leq N_{f}} \Gamma\left((X Y)^{-1} U^{J+K / 2} s_{i} s_{j}, X Y U^{J+K / 2} t_{i}^{-1} t_{j}^{-1} ; p, q\right) \\
& \times \prod_{J=0}^{\frac{K-1}{2}} \prod_{i=1}^{N_{f}} \Gamma\left((X Y)^{-1} U^{2 J+K / 2} s_{i}^{2}, X Y U^{2 J+K / 2} t_{i}^{-2} ; p, q\right) \\
& \times \prod_{i=1}^{N_{f}} \Gamma\left(U^{\frac{2-K}{2}} X^{K N_{f}+2} Y^{\frac{3 K N_{f}+4}{2}} s_{i}^{-1}, U^{\frac{2-K}{2}} X^{-\left(K N_{f}+2\right)} Y^{-\frac{3 K N_{f}+4}{2}} t_{i} ; p, q\right)
\end{aligned}
$$

where $U=(p q)^{\frac{1}{K+1}}, Y=(S T)^{1 / N_{f}}, S=\prod_{i=1}^{N_{f}} s_{i}, T=\prod_{i=1}^{N_{f}} t_{i}, X$ is an arbitrary parameter, and the balancing condition reads $U^{N-2} S T^{-1}=(p q)^{N_{f}}$. Again, the proof of the general equality $I_{E}=I_{M}$ is absent.

12.1.12. $S U\left(3 K N_{f}-5\right)$ with $N_{f} f+N_{f} \bar{f}+a d j+T_{A}+\bar{T}_{A}$. If we take $N=3 K N_{f}-5$ for $K$-odd in (9.18), we obtain the $s$-confinement [50]. The corresponding electric superconformal index is given by (9.19), and the magnetic superconformal index takes the form

$$
\begin{aligned}
I_{M}= & \prod_{L=0}^{K-1} \prod_{i, j=1}^{N_{f}} \Gamma\left(U^{L+K} s_{i} t_{j}^{-1}, U^{L} s_{i} t_{j}^{-1} ; p, q\right) \\
& \times \prod_{J=0}^{K-1} \prod_{1 \leq i<j \leq N_{f}} \Gamma\left((X Y)^{-1} U^{J+K / 2} s_{i} s_{j}, X Y U^{J+K / 2} t_{i}^{-1} t_{j}^{-1} ; p, q\right) \\
& \times \prod_{J=0}^{\frac{K-3}{2}} \prod_{i=1}^{N_{f}} \Gamma\left((X Y)^{-1} U^{2 J+1+K / 2} s_{i}^{2}, X Y U^{2 J+1+K / 2} t_{i}^{-2} ; p, q\right) \\
& \times \prod_{i=1}^{N_{f}} \Gamma\left(U^{\frac{2-K}{2}} X^{K N_{f}-2} Y^{\frac{3 K N_{f}-4}{2}} s_{i}^{-1}, U^{\frac{2-K}{2}} X^{-\left(K N_{f}-2\right)} Y^{-\frac{3 K N_{f}-4}{2}} t_{i} ; p, q\right),
\end{aligned}
$$

where $U=(p q)^{\frac{1}{K+1}}, Y=(S T)^{1 / N_{f}}, S=\prod_{i=1}^{N_{f}} s_{i}, T=\prod_{i=1}^{N_{f}} t_{i}, X$ is an arbitrary parameter, and the balancing condition reads $U^{N-2} S T^{-1}=(p q)^{N_{f}}$. No proof of the equality $I_{E}=I_{M}$ is known at present.

12.1.13. $S U(N)$ with $N_{f} f+\left(N_{f}-8\right) \bar{f}+a d j+T_{A}+\bar{T}_{S}$. If we set $N=3 K\left(N_{f}-4\right)-1$ in $(9.21)$, we obtain the $s$-confinement [50]. The corresponding electric superconformal index is given by 
(9.22), and

$$
\begin{aligned}
I_{M} & =\prod_{L=0}^{K-1} \prod_{i=1}^{N_{f}}\left[\prod_{j=1}^{N_{f}-8} \Gamma\left(U^{L+K} s_{i} t_{j}, U^{L} s_{i} t_{j} ; p, q\right) \Gamma\left((X Y)^{-1} U^{L+K / 2} s_{i}^{2} ; p, q\right)\right] \\
& \times \prod_{J=0}^{K-1}\left[\prod_{1 \leq i<j \leq N_{f}} \Gamma\left((X Y)^{-1} U^{J+K / 2} s_{i} s_{j} ; p, q\right) \prod_{1 \leq i<j \leq N_{f}-8} \Gamma\left(X Y U^{J+K / 2} t_{i} t_{j} ; p, q\right)\right] \quad(12.18) \\
& \times \prod_{i=1}^{N_{f}} \Gamma\left(U^{\frac{2-K}{2}} Y^{\frac{3 K\left(N_{f}-4\right)}{2}} s_{i}^{-1} ; p, q\right) \prod_{k=1}^{N_{f}-8} \Gamma\left(U^{\frac{2-K}{2}} Y^{-\frac{3 K\left(N_{f}-4\right)}{2}} t_{k}^{-1} ; p, q\right) \Gamma\left(U^{K / 2}\left(X Y^{N}\right)^{-1} ; p, q\right),
\end{aligned}
$$

where $U=(p q)^{\frac{1}{K+1}}$, the balancing condition reads $U^{N} X^{-4} Y^{-4} S T=(p q)^{N_{f}-4}$ with $S=$ $\prod_{i=1}^{N_{f}} s_{i}, T=\prod_{i=1}^{N_{f}-8} t_{i}$, and

$$
Y=X^{2}\left(S T^{-1}(p q)^{\frac{2(K-2)}{K+1}}\right)^{\frac{1}{N_{f}-4}} .
$$

Equality of indices defines another unproven elliptic beta integral evaluation.

12.1.14. New confining duality. Let us take the electric and magnetic $\mathcal{N}=1$ superconformal field theories described by the tables below

\begin{tabular}{|c|c|c|c|c|c|}
\hline & $S U(N+1)$ & $S P(2 N)$ & $S U(N+3)$ & $U(1)$ & $U(1)_{R}$ \\
\hline$Q_{1}$ & $\bar{f}$ & 1 & $f$ & 1 & 0 \\
$Q_{2}$ & $f$ & $f$ & 1 & $-\frac{N+3}{2}$ & 1 \\
$X$ & $\bar{T}_{A}$ & 1 & 1 & $N+3$ & 0 \\
\hline \hline$q_{1}=Q_{1}^{N+1}$ & & 1 & $\bar{T}_{A}$ & $N+1$ & 0 \\
$q_{2}=Q_{1} Q_{2}$ & & $f$ & $f$ & $-\frac{N+1}{2}$ & 1 \\
\hline
\end{tabular}

The dynamically generated superpotential in this case is $W_{d y n} \propto Q_{1}^{N+1}\left(Q_{1} Q_{2}\right)^{2}$.

The indices read

$$
\begin{aligned}
I_{E}= & \frac{(p ; p)_{\infty}^{N}(q ; q)_{\infty}^{N}}{(N+1) !} \int_{\mathbb{T}^{N}} \prod_{1 \leq i<j \leq N+1} \frac{\Gamma\left(S z_{i}^{-1} z_{j}^{-1} ; p, q\right)}{\Gamma\left(z_{i} z_{j}^{-1}, z_{i}^{-1} z_{j} ; p, q\right)} \\
& \times \prod_{j=1}^{N+1} \frac{\prod_{k=1}^{N} \Gamma\left(t_{k} z_{j} ; p, q\right) \prod_{m=1}^{N+3} \Gamma\left(s_{m} z_{j}^{-1} ; p, q\right)}{\prod_{k=1}^{N} \Gamma\left(S t_{k} z_{j}^{-1} ; p, q\right)} \prod_{j=1}^{N} \frac{d z_{j}}{2 \pi \mathrm{i} z_{j}}
\end{aligned}
$$

where $\prod_{j=1}^{N+1}=1$, and

$$
I_{M}=\prod_{k=1}^{N} \prod_{m=1}^{N+3} \frac{\Gamma\left(t_{k} s_{m} ; p, q\right)}{\Gamma\left(S t_{k} s_{m}^{-1} ; p, q\right)} \prod_{1 \leq l<m \leq N+3} \Gamma\left(S s_{l}^{-1} s_{m}^{-1} ; p, q\right)
$$

with the balancing condition $S=\prod_{m=1}^{N+3} s_{m}$.

The elliptic beta integral described by the equality $I_{E}=I_{M}$ was discovered by the first author and Warnaar in [93]. Here it defines a new pair of $\mathcal{N}=1$ supersymmetric quantum field theories dual to each other, which was not considered earlier in the literature. Moreover, it gives a counterexample to the classification of $s$-confining theories in [15]. Conjecturally, there exists a symmetry transformation for a higher order generalization of $I_{E}$ depending on the bigger number of parameters. Correspondingly, there should exist a more complicated Seiberg duality as well. 
ELLIPTIC HYPERGEOMETRY OF SUPERSYMMETRIC DUALITIES

\subsection{Exceptional cases for unitary gauge groups.}

12.2.1. $S U(6)$ with $4 f+4 \bar{f}$. The following pair of models was constructed in [16]:

\begin{tabular}{|c|c|c|c|c|c|c|}
\hline & $S U(6)$ & $S U(4)$ & $S U(4)$ & $U(1)_{1}$ & $U(1)_{2}$ & $U(1)_{R}$ \\
\hline$Q$ & $f$ & $f$ & 1 & 1 & 3 & 1 \\
$\widetilde{Q}$ & $\bar{f}$ & 1 & $f$ & -1 & 3 & 1 \\
$A$ & $T_{3 A}$ & 1 & 1 & 0 & -4 & -1 \\
\hline \hline$M_{0}=Q \widetilde{Q}$ & & $f$ & $f$ & 0 & 6 & 2 \\
$M_{2}=Q A^{2} \widetilde{Q}$ & & $f$ & $f$ & 0 & -2 & 0 \\
$B_{1}=A Q^{3}$ & & $\bar{f}$ & $\frac{1}{f}$ & 3 & 5 & 2 \\
$\widetilde{B}_{1}=A \widetilde{Q}^{3}$ & & $\frac{1}{f}$ & $\frac{1}{f}$ & 3 & -3 & 0 \\
$B_{3}=A^{3} Q^{3}$ & & $\frac{f}{2}$ & -3 & 0 \\
$\widetilde{B}_{3}=A^{3} \widetilde{Q}^{3}$ & & 1 & $\frac{1}{2}$ & 0 & -16 & -4 \\
$T=A^{4}$ & & 1 & 1 &
\end{tabular}

Their superconformal indices read

$$
\begin{aligned}
& I_{E}= \frac{(p ; p)_{\infty}^{5}(q ; q)_{\infty}^{5}}{6 !} \int_{\mathbb{T}^{5}} \frac{\prod_{1 \leq i<j<k \leq 6} \Gamma\left(U z_{i} z_{j} z_{k} ; p, q\right)}{\prod_{1 \leq i<j \leq 6} \Gamma\left(z_{i} z_{j}^{-1}, z_{i}^{-1} z_{j} ; p, q\right)} \\
& \times \prod_{j=1}^{6} \prod_{k=1}^{4} \Gamma\left(s_{k} z_{j}, t_{k} z_{j}^{-1} ; p, q\right) \prod_{j=1}^{5} \frac{d z_{j}}{2 \pi \mathrm{i} z_{j}},
\end{aligned}
$$

where $\prod_{j=1}^{6} z_{j}=1$, and

$$
\begin{aligned}
I_{M}=\Gamma\left(U^{4} ; p, q\right) \prod_{k, l=1}^{4} \Gamma\left(s_{k} t_{l}, U^{2} s_{k} t_{l} ; p, q\right) \\
\quad \times \prod_{k=1}^{4} \Gamma\left(S U s_{k}^{-1}, S U^{3} s_{k}^{-1}, T U t_{k}^{-1}, T U^{3} t_{k}^{-1} ; p, q\right)
\end{aligned}
$$

with $S=\prod_{k=1}^{4} s_{k}, T=\prod_{k=1}^{4} t_{k}$, and the balancing condition $S T U^{6}=p q$.

There is actually a lift of this duality to interacting magnetic theories found in [17]. The theory is self-dual and is based on $S U(6)$ gauge group and the flavor symmetry group is

$$
F=S U(6) \times S U(6) \times U(1)_{1} \times U(1)_{1} .
$$

The matter content of the dual theories is given in the following tables: the electric theory

\begin{tabular}{|c|c|c|c|c|c|c|}
\hline & $S U(6)$ & $S U(6)$ & $S U(6)$ & $U(1)_{1}$ & $U(1)_{2}$ & $U(1)_{R}$ \\
\hline$Q$ & $f$ & $f$ & 1 & 1 & 1 & $\frac{1}{2}$ \\
$\bar{Q}$ & $\bar{f}$ & 1 & $f$ & -1 & 1 & $\frac{1}{2}$ \\
$A$ & $T_{3 A}$ & 1 & 1 & 0 & -2 & 0 \\
\hline
\end{tabular}

and the magnetic theory

\begin{tabular}{|c|c|c|c|c|c|c|}
\hline & $S U(6)$ & $S U(6)$ & $S U(6)$ & $U(1)_{1}$ & $U(1)_{2}$ & $U(1)_{R}$ \\
\hline$q$ & $f$ & $\bar{f}$ & $\frac{1}{f}$ & 1 & 1 & $\frac{1}{2}$ \\
$\bar{q}$ & $\bar{f}$ & 1 & -1 & 1 & $\frac{1}{2}$ \\
$a$ & $T_{3 A}$ & 1 & 1 & 0 & -2 & 0 \\
$M_{0}$ & 1 & $f$ & $f$ & 0 & 2 & 1 \\
$M_{2}$ & 1 & $f$ & $f$ & 0 & -2 & 1 \\
\hline
\end{tabular}


The electric superconformal index is

$$
\begin{gathered}
I_{E}=\frac{(p ; p)_{\infty}^{5}(q ; q)_{\infty}^{5}}{6 !} \int_{\mathbb{T}^{5}} \frac{\prod_{1 \leq i<j<k \leq 6} \Gamma\left(U z_{i} z_{j} z_{k} ; p, q\right)}{\prod_{1 \leq i<j \leq 6} \Gamma\left(z_{i} z_{j}^{-1}, z_{i}^{-1} z_{j} ; p, q\right)} \\
\quad \times \prod_{i=1}^{6} \prod_{j=1}^{6} \Gamma\left(s_{i} z_{j}, t_{i} z_{j}^{-1} ; p, q\right) \prod_{j=1}^{5} \frac{d z_{j}}{2 \pi \mathrm{i} z_{j}}
\end{gathered}
$$

and the magnetic index is

$$
\begin{aligned}
I_{M}= & \frac{(p ; p)_{\infty}^{5}(q ; q)_{\infty}^{5}}{6 !} \prod_{i, j=1}^{6} \Gamma\left(s_{i} t_{j}, U^{2} s_{i} t_{j} ; p, q\right) \\
& \times \int_{\mathbb{T}^{5}} \frac{\prod_{1 \leq i<j<k \leq 6} \Gamma\left(U z_{i} z_{j} z_{k} ; p, q\right)}{\prod_{1 \leq i<j \leq 6} \Gamma\left(z_{i} z_{j}^{-1}, z_{i}^{-1} z_{j} ; p, q\right)} \prod_{i=1}^{6} \prod_{j=1}^{6} \Gamma\left(\frac{\sqrt[3]{S}}{s_{i}} z_{j}, \frac{\sqrt[3]{T}}{t_{i}} z_{j}^{-1} ; p, q\right) \prod_{j=1}^{5} \frac{d z_{j}}{2 \pi \mathrm{i} z_{j}}
\end{aligned}
$$

where $S=\prod_{i=1}^{6}, T=\prod_{j=1}^{6} t_{j}$, and the balancing condition reads $S T U^{6}=(p q)^{3}$.

12.2.2. $S U(5)$ with $3 T_{A}+3 \bar{f}$. Models [16]:

\begin{tabular}{|c|c|c|c|c|c|}
\hline & $S U(5)$ & $S U(3)$ & $S U(3)$ & $U(1)$ & $U(1)_{R}$ \\
\hline$Q$ & $\bar{f}$ & 1 & $f$ & -3 & $\frac{2}{3}$ \\
$A$ & $T_{A}$ & $f$ & 1 & 1 & 0 \\
\hline \hline$A Q^{2}$ & & $f$ & $\bar{f}$ & -5 & $\frac{4}{3}$ \\
$A^{3} Q$ & & $T_{A S}$ & $f$ & 0 & $\frac{2}{3}$ \\
$A^{5}$ & & $T_{S}$ & 1 & 5 & 0 \\
\hline
\end{tabular}

Indices:

$$
I_{E}=\frac{(p ; p)_{\infty}^{4}(q ; q)_{\infty}^{4}}{5 !} \int_{\mathbb{T}^{4}} \prod_{1 \leq i<j \leq 5} \frac{\prod_{k=1}^{3} \Gamma\left(s_{k} z_{i} z_{j} ; p, q\right)}{\Gamma\left(z_{i} z_{j}^{-1}, z_{i}^{-1} z_{j} ; p, q\right)} \prod_{j=1}^{5} \prod_{k=1}^{3} \Gamma\left(t_{k} z_{j}^{-1} ; p, q\right) \prod_{j=1}^{4} \frac{d z_{j}}{2 \pi \mathrm{i} z_{j}}
$$

with $\prod_{j=1}^{5} z_{j}=1$, and

$$
\begin{aligned}
I_{M}= & \prod_{k, l=1}^{3} \Gamma\left(T s_{k} t_{l}^{-1} ; p, q\right) \prod_{1 \leq j<k \leq 3} \Gamma\left(S s_{j} s_{k} ; p, q\right) \\
& \times \prod_{j=1}^{3} \Gamma\left(S s_{j}^{2} ; p, q\right) \prod_{k, j, l=1 ; k \neq j}^{3} \Gamma\left(s_{k}^{2} s_{j} t_{l} ; p, q\right) \prod_{l=1}^{3} \Gamma\left(S t_{l} ; p, q\right)^{2},
\end{aligned}
$$

where $S=\prod_{k=1}^{3} s_{k}, T=\prod_{k=1}^{3} t_{k}$, and the balancing condition reads $S^{3} T=p q$.

12.2.3. $S U(5)$ with $2 T_{A}+4 \bar{f}+2 f$. Models [16]: 
ELLIPTIC HYPERGEOMETRY OF SUPERSYMMETRIC DUALITIES

\begin{tabular}{|c|c|c|c|c|c|c|c|}
\hline & $S U(5)$ & $S U(2)$ & $S U(4)$ & $S U(2)$ & $U(1)_{1}$ & $U(1)_{2}$ & $U(1)_{R}$ \\
\hline$Q$ & $f$ & 1 & 1 & $f$ & -2 & 1 & $\frac{1}{3}$ \\
$\widetilde{Q}$ & $\widetilde{f}$ & 1 & $f$ & 1 & 1 & 1 & $\frac{1}{3}$ \\
$A$ & $T_{A}$ & $f$ & 1 & 1 & 0 & -1 & 0 \\
\hline \hline$Q \widetilde{Q}$ & & 1 & $f$ & $f$ & -1 & 2 & $\frac{2}{3}$ \\
$A \widetilde{Q}$ & & $f$ & $T_{A}$ & 1 & 2 & 1 & $\frac{2}{3}$ \\
$A^{2} Q$ & & $T_{S}$ & 1 & $f$ & -2 & -1 & $\frac{1}{3}$ \\
$A^{3} \widetilde{Q}$ & & $f$ & $f$ & 1 & 1 & -2 & $\frac{1}{3}$ \\
$A^{2} Q^{2} \widetilde{Q}$ & & 1 & $f$ & 1 & -3 & 1 & 1 \\
\hline
\end{tabular}

Indices:

$$
\begin{gathered}
I_{E}=\frac{(p ; p)_{\infty}^{4}(q ; q)_{\infty}^{4}}{5 !} \int_{\mathbb{T}^{4}} \prod_{1 \leq i<j \leq 5} \frac{\prod_{k=1}^{2} \Gamma\left(s_{k} z_{i} z_{j} ; p, q\right)}{\Gamma\left(z_{i} z_{j}^{-1}, z_{i}^{-1} z_{j} ; p, q\right)} \\
\times \prod_{j=1}^{5} \prod_{k=1}^{4} \Gamma\left(t_{k} z_{j}^{-1}, u_{k} z_{j} ; p, q\right) \prod_{j=1}^{4} \frac{d z_{j}}{2 \pi \mathrm{i} z_{j}}
\end{gathered}
$$

with $\prod_{j=1}^{5} z_{j}=1$, and

$$
\begin{aligned}
I_{M}= & \prod_{k=1}^{4} \Gamma\left(S U t_{k} ; p, q\right) \prod_{k=1}^{4} \prod_{l=1}^{2} \Gamma\left(t_{k} u_{l}, S t_{k} s_{l} ; p, q\right) \prod_{k=1}^{2} \Gamma\left(S u_{k} ; p, q\right) \\
& \times \prod_{k, l=1}^{2} \Gamma\left(s_{l}^{2} u_{k} ; p, q\right) \prod_{k=1}^{2} \prod_{1 \leq l<m \leq 4} \Gamma\left(s_{k} t_{l} t_{m} ; p, q\right),
\end{aligned}
$$

where the balancing condition reads $S^{3} T U=p q$ and $S=\prod_{k=1}^{2} s_{k}, T=\prod_{k=1}^{4} t_{k}, U=u_{1} u_{2}$.

12.2.4. $S U(6)$ with $2 T_{A}+f+5 \bar{f}$. Models [16]:

\begin{tabular}{|c|c|c|c|c|c|c|}
\hline & $S U(6)$ & $S U(2)$ & $S U(5)$ & $U(1)_{1}$ & $U(1)_{2}$ & $U(1)_{R}$ \\
\hline$Q$ & $f$ & 1 & 1 & -5 & -4 & 0 \\
$\widetilde{Q}$ & $\bar{f}$ & 1 & $f$ & 1 & -4 & 0 \\
$A$ & $T_{A}$ & $f$ & 1 & 0 & 3 & $\frac{1}{4}$ \\
\hline \hline$Q \widetilde{Q}$ & & 1 & $f$ & -4 & -8 & 0 \\
$A \widetilde{Q}$ & & $f$ & $T_{A}$ & 2 & -5 & $\frac{1}{4}$ \\
$A^{3}$ & & $T_{3 S}$ & 1 & 0 & 9 & $\frac{3}{4}$ \\
$A^{3} Q \widetilde{Q}$ & & $f$ & $f$ & -4 & 1 & $\frac{3}{4}$ \\
$A^{4} \widetilde{Q}^{2}$ & & 1 & $T_{A}$ & 2 & 4 & 1 \\
\hline
\end{tabular}

Indices:

$$
\begin{aligned}
I_{E}= & \frac{(p ; p)_{\infty}^{5}(q ; q)_{\infty}^{5}}{6 !} \int_{\mathbb{T}^{5}} \prod_{1 \leq i<j \leq 6} \frac{\Gamma\left(U z_{i} z_{j} ; p, q\right)}{\Gamma\left(z_{i} z_{j}^{-1}, z_{i}^{-1} z_{j} ; p, q\right)} \\
& \times \prod_{l=1}^{2} \prod_{1 \leq j<k \leq 6} \Gamma\left(s_{l} z_{j} z_{k} ; p, q\right) \prod_{j=1}^{6} \prod_{k=1}^{5} \Gamma\left(t_{k} z_{j}^{-1} ; p, q\right) \prod_{j=1}^{5} \frac{d z_{j}}{2 \pi \mathrm{i} z_{j}}
\end{aligned}
$$


with $\prod_{i=1}^{6} z_{i}=1$, and

$$
\begin{aligned}
I_{M}= & \prod_{k}^{5} \Gamma\left(U t_{k} ; p, q\right) \prod_{k=1}^{2} \prod_{j=1}^{5} \Gamma\left(S U s_{k} t_{j} ; p, q\right) \prod_{k=1}^{2} \prod_{1 \leq j<l \leq 5} \Gamma\left(s_{k} t_{j} t_{l} ; p, q\right) \\
& \times \prod_{1 \leq j<k \leq 5} \Gamma\left(S^{2} t_{j} t_{k} ; p, q\right) \prod_{j=1}^{2} \Gamma\left(s_{j}^{3}, S s_{j} ; p, q\right),
\end{aligned}
$$

where the balancing condition reads $S^{4} T U=p q$ and $S=\prod_{k=1}^{2} s_{k}, T=\prod_{k=1}^{5} t_{k}$.

12.2.5. $S U(7)$ with $2 T_{A}+6 \bar{f}$. Models [16]:

Indices:

\begin{tabular}{|c|c|c|c|c|c|}
\hline & $S U(7)$ & $S U(2)$ & $S U(6)$ & $U(1)$ & $U(1)_{R}$ \\
\hline$Q$ & $\bar{f}$ & 1 & $f$ & -5 & $\frac{1}{3}$ \\
$A$ & $T_{A}$ & $f$ & 1 & 3 & 0 \\
\hline \hline$A Q^{2}$ & & $f$ & $T_{A}$ & -7 & $\frac{2}{3}$ \\
$A^{4} Q$ & & $T_{S}$ & $f$ & 7 & $\frac{1}{3}$ \\
\hline
\end{tabular}

$$
\begin{aligned}
I_{E}= & \frac{(p ; p)_{\infty}^{6}(q ; q)_{\infty}^{6}}{7 !} \int_{\mathbb{T}^{6}} \prod_{1 \leq i<j \leq 7} \frac{1}{\Gamma\left(z_{i} z_{j}^{-1}, z_{i}^{-1} z_{j} ; p, q\right)} \\
& \times \prod_{k=1}^{2} \prod_{1 \leq i<j \leq 7} \Gamma\left(s_{k} z_{i} z_{j} ; p, q\right) \prod_{k=1}^{6} \prod_{j=1}^{7} \Gamma\left(t_{k} z_{j}^{-1} ; p, q\right) \prod_{j=1}^{6} \frac{d z_{j}}{2 \pi \mathrm{i} z_{j}},
\end{aligned}
$$

with $\prod_{i=1}^{7} z_{i}=1$, and

$$
I_{M}=\prod_{k=1}^{6} \Gamma\left(S^{2} t_{k} ; p, q\right) \prod_{k=1}^{6} \prod_{l=1}^{2} \Gamma\left(S s_{l}^{2} t_{k} ; p, q\right) \prod_{k=1}^{2} \prod_{1 \leq l<m \leq 6} \Gamma\left(s_{k} t_{l} t_{m} ; p, q\right),
$$

where the balancing condition reads $S^{5} T=p q$ and $S=\prod_{k=1}^{2} s_{k}, T=\prod_{k=1}^{6} t_{k}$.

All the equalities of superconformal indices of dual theories, $I_{E}=I_{M}$, described in this section represent new elliptic beta integrals requiring a rigorous proof (the parameter values are assumed to guarantee that only sequences of poles of the integrands converging to zero are located inside the contour $\mathbb{T}$ ).

\subsection{Symplectic gauge group.}

12.3.1. $S P(2 N)$ with $(2 N+4) f$. Models [43]:

\begin{tabular}{|c|c|c|c|}
\hline & $S P(2 N)$ & $S U(2 N+4)$ & $U(1)_{R}$ \\
\hline$Q$ & $f$ & $f$ & $2 r=\frac{1}{N+2}$ \\
\hline \hline$Q^{2}$ & & $T_{A}$ & $2 r=\frac{2}{N+2}$ \\
\hline
\end{tabular}

Indices:

$$
I_{E}=\frac{(p ; p)_{\infty}^{N}(q ; q)_{\infty}^{N}}{2^{N} N !} \int_{\mathbb{T}^{N}} \prod_{1 \leq i<j \leq N} \frac{1}{\Gamma\left(z_{i}^{ \pm 1} z_{j}^{ \pm 1} ; p, q\right)} \prod_{j=1}^{N} \frac{\prod_{m=1}^{2 N+4} \Gamma\left(t_{m} z_{j}^{ \pm 1} ; p, q\right)}{\Gamma\left(z_{j}^{ \pm 2} ; p, q\right)} \frac{d z_{j}}{2 \pi \mathrm{i} z_{j}}
$$

and

$$
I_{M}=\prod_{1 \leq m<s \leq 2 N+4} \Gamma\left(t_{m} t_{s} ; p, q\right),
$$

where the balancing condition reads $\prod_{m=1}^{2 N+4} t_{m}=p q$. 
The equality $I_{E}=I_{M}$ was introduced and partially justified by van Diejen and the first author in [19] and completely proven in [65] and [83]. Its simplest $p \rightarrow 0$ limit yields one of the Gustafson $q$-beta integrals [35].

12.3.2. $S P(2 N)$ with $6 f$ and $T_{A}$. This duality was considered in $[12,18]$. The flavor symmetry group is $F=S U(6) \times U(1)$ and the field content is

\begin{tabular}{|c|c|c|c|c|}
\hline & $S P(2 N)$ & $S U(6)$ & $U(1)$ & $U(1)_{R}$ \\
\hline$Q$ & $f$ & $f$ & $N-1$ & $2 r=\frac{1}{3}$ \\
$A$ & $T_{A}$ & 1 & -3 & 0 \\
\hline \hline$A^{k}$ & & 1 & $-3 k$ & 0 \\
$Q A^{m} Q$ & & $T_{A}$ & $2(N-1)-3 m$ & $\frac{2}{3}$ \\
\hline
\end{tabular}

where $k=2, \ldots, N$ and $m=0, \ldots, N-1$.

The electric superconformal index is given by the integral

$$
\begin{gathered}
I_{E}=\frac{(p ; p)_{\infty}^{N}(q ; q)_{\infty}^{N} \Gamma(t ; p, q)^{N-1} \int_{\mathbb{T}^{N}} \prod_{1 \leq i<j \leq N} \frac{\Gamma\left(t z_{i}^{ \pm 1} z_{j}^{ \pm 1} ; p, q\right)}{\Gamma\left(z_{i}^{ \pm 1} z_{j}^{ \pm 1} ; p, q\right)}}{2^{N} N !} \\
\quad \times \prod_{j=1}^{N} \frac{\prod_{m=1}^{6} \Gamma\left(t_{m} z_{j}^{ \pm 1} ; p, q\right)}{\Gamma\left(z_{j}^{ \pm 2} ; p, q\right)} \prod_{j=1}^{N} \frac{d z_{j}}{2 \pi \mathrm{i} z_{j}}
\end{gathered}
$$

and the magnetic index is

$$
I_{M}=\prod_{j=2}^{N} \Gamma\left(t^{j} ; p, q\right) \prod_{j=1}^{N} \prod_{1 \leq m<s \leq 6} \Gamma\left(t^{j-1} t_{m} t_{s} ; p, q\right),
$$

where the balancing condition reads $t^{2 N-2} \prod_{m=1}^{6} t_{m}=p q$.

The equality $I_{E}=I_{M}$ coincides with the elliptic Selberg integral suggested by van Diejen and the first author in [19] and proven in [20] as a consequence of the $B C_{n}$-elliptic beta integral of type I (its direct proof is given also in [65]). The Selberg integral plays a fundamental role in mathematics and mathematical physics because of a large number of applications [30]. Note that this exactly computable integral gives a confirmation of the KS duality for the special values of parameters $N_{f}=3, K=N$.

\begin{tabular}{|c|c|c|c|c|c|c|c|c|}
\hline & $S P(2 M)$ & $S U(4)$ & $S P\left(2 l_{1}\right)$ & $S P\left(2 l_{2}\right)$ & $\ldots$ & $S P\left(2 l_{K}\right)$ & $U(1)$ & $U(1)_{R}$ \\
\hline$W_{1}$ & $f$ & $\bar{f}$ & 1 & 1 & $\ldots$ & 1 & $-\frac{M-2}{4}$ & 0 \\
\hline$Q_{1}$ & $f$ & 1 & $f$ & 1 & $\ldots$ & 1 & $-\frac{n_{1}}{2}$ & 1 \\
\hline$Q_{1}$ & $f$ & 1 & 1 & $f$ & 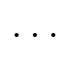 & 1 & $-\frac{n_{2}}{2}$ & 1 \\
\hline$\ddot{Q}_{K}$ & $f$ & 1 & 1 & 1 & . . & $f$ & $-\frac{n_{K}}{2}$ & 1 \\
\hline$X$ & $\bar{T}_{A}$ & 1 & 1 & 1 & $\ldots$ & 1 & $1^{2}$ & 0 \\
\hline$W_{1}^{2} X^{j}$ & & 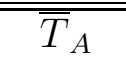 & 1 & 1 & $\ldots$ & 1 & = $j-\frac{M-2}{2}$ & 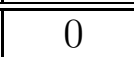 \\
\hline$W_{1} Q_{1} X^{k_{1}}$ & & $\bar{f}$ & $f$ & 1 & $\ldots$ & 1 & $-\frac{M-2}{4}-\frac{n_{1}}{2}+k_{1}$ & 1 \\
\hline$W_{1} Q_{2} X^{k_{2}}$ & & $\bar{f}$ & 1 & $f$ & $\ldots$ & 1 & $-\frac{M-2}{4}-\frac{n_{2}}{2}+k_{2}$ & 1 \\
\hline$W_{1} Q_{K} X^{k_{K}}$ & & $\bar{f}$ & 1 & 1 & & $f$ & $-\frac{M-2}{4}-\frac{n_{K}}{2}+k_{K}$ & 1 \\
\hline
\end{tabular}

12.3.3. $S P(2 M)+4 \bar{f}+2 M f+T_{A}$. This new confining duality is obtained from the results of Sect. 7 by formal setting $N=0$. The models are described in the table 
where $j=0, \ldots, M-1, k_{i}=0, \ldots, n_{i}-1$ for any $i=1, \ldots, K, n_{1} \neq n_{2} \neq \ldots \neq n_{K}$ and $\sum_{i=1}^{K} l_{i} n_{i}=M$.

The superconformal indices have the form

$$
\begin{aligned}
I_{E}= & \frac{(p ; p)_{\infty}^{M}(q ; q)_{\infty}^{M}}{2^{M} M !} \Gamma(t ; p, q)^{M-1} \int_{\mathbb{T}^{M}} \prod_{1 \leq i<j \leq M} \frac{\Gamma\left(t z_{i}^{ \pm 1} z_{j}^{ \pm 1} ; p, q\right)}{\Gamma\left(z_{i}^{ \pm 1} z_{j}^{ \pm 1} ; p, q\right)} \\
& \times \prod_{j=1}^{M} \frac{\prod_{k=1}^{4} \Gamma\left(t t_{k}^{-1} z_{j}^{ \pm 1} ; p, q\right) \prod_{r=1}^{K} \prod_{m=1}^{l_{r}} \Gamma\left(s_{r, m} z_{j}^{ \pm 1} ; p, q\right)}{\Gamma\left(z_{j}^{ \pm 2} ; p, q\right) \prod_{r=1}^{K} \prod_{m=1}^{l_{r}} \Gamma\left(t^{n_{r}} s_{r, m} z_{j}^{ \pm 1} ; p, q\right)} \frac{d z_{j}}{2 \pi \mathrm{i} z_{j}}
\end{aligned}
$$

and

$$
I_{M}=\prod_{i=0}^{M-1} \prod_{1 \leq k<r \leq 4} \Gamma\left(t^{i+2} t_{k}^{-1} t_{r}^{-1} ; p, q\right) \prod_{k=1}^{4} \prod_{r=1}^{K} \prod_{i=1}^{l_{r}} \prod_{k_{m}=0}^{n_{r}-1} \frac{\Gamma\left(t^{k_{m}+1} t_{k}^{-1} s_{r, i} ; p, q\right)}{\Gamma\left(t^{k_{m}} t_{k} s_{r, i} ; p, q\right)}
$$

where the balancing condition is $\prod_{r=1}^{4} t_{r}=t^{2+M}$. The equality $I_{E}=I_{M}$ was conjectured in [68] and proven in [9]. This duality gives another example of $s$-confining theories missed in [15].

12.3.4. $S P\left(2 K\left(N_{f}-2\right)\right)$ with $N_{f} f+T_{A}$. This duality was considered in $[14,50]$. From (10.1) we see that the choice $N=K\left(N_{f}-2\right)$ yields $\widetilde{N}=0$, and the theory is $s$-confining. The field content of the electric and magnetic theories is easily found from the tables given in Sect. 10.1. For brevity we skip the electric superconformal index given by (10.2), and present directly the magnetic index

$$
I_{M}=\Gamma(U ; p, q)^{-1} \prod_{l=1}^{K} \prod_{1 \leq i<j \leq 2 N_{f}} \Gamma\left(U^{l-1} s_{i} s_{j} ; p, q\right),
$$

where $U=(p q)^{\frac{1}{K+1}}$ and the balancing condition reads $U^{2 K N_{f}-2 K} \prod_{i=1}^{2 N_{f}} s_{i}=(p q)^{N_{f}}$. The conjecture $I_{E}=I_{M}$ represents a new elliptic beta integral. For $K=1$ it reduces to the proven relation of Sect. 12.3.1.

12.3.5. $S P\left(2\left(N_{f}-2+2 K N_{f}\right)\right)$ with $N_{f} f+T_{S}$. Looking at (10.4) and fixing $N=N_{f}-2+2 K N_{f}$, we obtain the $s$-confining theory which was considered in $[14,50]$. The corresponding electric superconformal index is given by (10.5), and the magnetic index takes the form

$$
\begin{aligned}
I_{M}= & \prod_{l=0}^{K} \prod_{1 \leq i<j \leq 2 N_{f}} \Gamma\left(U^{l} s_{i} s_{j} ; p, q\right) \\
& \times \prod_{l=0}^{K-1} \prod_{1 \leq i<j \leq 2 N_{f}} \Gamma\left(U^{(2 l+1) / 2} s_{i} s_{j} ; p, q\right) \prod_{i=1}^{2 N_{f}} \Gamma\left(U^{(2 l+1) / 2} s_{i}^{ \pm 2} ; p, q\right),
\end{aligned}
$$

where $U=(p q)^{\frac{1}{K+1}}$ and the balancing condition reads $U^{2 N_{f}-2+4 K N_{f}} \prod_{i=1}^{2 N_{f}} s_{i}=(p q)^{N_{f}}$. The conjecture $I_{E}=I_{M}$ represents a new elliptic beta integral.

12.3.6. $S P\left(2\left(3 K N_{f}-4 K-2\right)\right)$ with $N_{f} f+2 T_{A}$. Looking at (10.7) and fixing $N=3 K N_{f}-4 K-$ 2 for odd $K$, we obtain the $s$-confining theory which was considered in [50]. The corresponding electric superconformal index is given by (10.8), and the magnetic index takes the form

$$
I_{M}=\Gamma\left(U, U^{\frac{K}{2}} ; p, q\right)^{-1} \prod_{J=0}^{K-1} \prod_{L=0}^{2} \prod_{1 \leq i<j \leq 2 N_{f}} \Gamma\left(U^{J+\frac{K L}{2}} s_{i} s_{j} ; p, q\right) \prod_{J=0}^{\frac{K-1}{2}} \prod_{j=1}^{2 N_{f}} \Gamma\left(U^{2 J+1+\frac{K}{2}} s_{j}^{2} ; p, q\right)
$$


where $U=(p q)^{\frac{1}{K+1}}$ and the balancing condition reads $U^{3 K N_{f}-2 K-1} \prod_{i=1}^{2 N_{f}} s_{i}=(p q)^{N_{f}}$. Rigorous justification of the expected equality $I_{E}=I_{M}$ is absent at the moment.

12.3.7. $S P\left(2\left(3 K N_{f}-4 K+2\right)\right)$ with $N_{f} f+T_{S}+T_{A}$. Looking at (10.10) and fixing $N=$ $3 K N_{f}-4 K+2$ for $K$ odd, we obtain the $s$-confining theory which was considered in [50]. The corresponding electric superconformal index is given by (10.11), and the magnetic index has the form

$$
I_{M}=\Gamma(U ; p, q)^{-1} \prod_{J=0}^{K-1} \prod_{L=0}^{2} \prod_{1 \leq i<j \leq 2 N_{f}} \Gamma\left(U^{J+\frac{K L}{2}} s_{i} s_{j} ; p, q\right) \prod_{J=0}^{\frac{K-1}{2}} \prod_{j=1}^{2 N_{f}} \Gamma\left(U^{2 J+\frac{K}{2}} s_{j}^{2} ; p, q\right),
$$

where $U=(p q)^{\frac{1}{K+1}}$ and the balancing condition reads $U^{3 K N_{f}-2 K+1} \prod_{i=1}^{2 N_{f}} s_{i}=(p q)^{N_{f}}$. The conjectural equality $I_{E}=I_{M}$ is our last new elliptic beta integral for classical root systems.

\section{Exceptional $G_{2}$ Group}

$G_{2}$ with 5 flavors. This s-confining duality was discussed in [33, 60]. The electric theory with the gauge group $G_{2}$ and its magnetic dual are described in the table below

\begin{tabular}{|c|c|c|c|}
\hline & $G_{2}$ & $S U(5)$ & $U(1)_{R}$ \\
\hline$Q$ & 7 & $f$ & $2 r=\frac{1}{5}$ \\
\hline \hline$Q^{2}$ & & $T_{S}$ & $\frac{2}{5}$ \\
$Q^{3}$ & & $\bar{T}_{A}$ & $\frac{3}{5}$ \\
$Q^{4}$ & & $\bar{f}$ & $\frac{4}{5}$ \\
\hline
\end{tabular}

The superconformal indices are

$$
I_{E}=\frac{(p ; p)_{\infty}^{2}(q ; q)_{\infty}^{2}}{2^{2} 3} \prod_{m=1}^{5} \Gamma\left(t_{m} ; p, q\right) \int_{\mathbb{T}^{2}} \frac{\prod_{k=1}^{3} \prod_{m=1}^{5} \Gamma\left(t_{m} z_{k}^{ \pm 1} ; p, q\right)}{\prod_{1 \leq j<k \leq 3} \Gamma\left(z_{j}^{ \pm 1} z_{k}^{ \pm 1} ; p, q\right)} \prod_{j=1}^{2} \frac{d z_{j}}{2 \pi \mathrm{i} z_{j}}
$$

where $z_{1} z_{2} z_{3}=1,\left|t_{m}\right|<1$, and

$$
I_{M}=\prod_{m=1}^{5} \frac{\Gamma\left(t_{m}^{2} ; p, q\right)}{\Gamma\left((p q)^{1 / 2} t_{m} ; p, q\right)} \prod_{1 \leq l<m \leq 5} \frac{\Gamma\left(t_{l} t_{m} ; p, q\right)}{\Gamma\left((p q)^{1 / 2} t_{l} t_{m} ; p, q\right)}
$$

with the balancing condition $\prod_{m=1}^{5} t_{m}=(p q)^{1 / 2}$.

The conjecture $I_{E}=I_{M}$ describes the first elliptic beta integral for exceptional root systems (it was mentioned in [91] and proposed also earlier by M. Ito). Substituting $t_{5}=$ $(p q)^{1 / 2} /\left(t_{1} t_{2} t_{3} t_{4}\right)$ in (13.1) and (13.2), and taking the limit $p \rightarrow 0$, one obtains the four parameter $q$-beta integral on the $G_{2}$ root system derived in [36].

$G_{2}$ with $5<N_{f}<12$ flavors. This duality was discovered by Pouliot in [61]. The electric theory has gauge group $G_{2}$, but its magnetic dual has $S U\left(N_{f}-3\right)$ gauge group. Their field content is presented in the tables below.

The electric theory (the vector superfield $V$ is omitted):

\begin{tabular}{|c|c|c|c|}
\hline & $G_{2}$ & $S U\left(N_{f}\right)$ & $U(1)_{R}$ \\
\hline$Q$ & 7 & $f$ & $2 r=1-\frac{4}{N_{f}}$ \\
\hline
\end{tabular}

and the magnetic theory (the vector superfield $\widetilde{V}$ is omitted): 


\begin{tabular}{|c|c|c|c|}
\hline & $S U\left(N_{f}-3\right)$ & $S U\left(N_{f}\right)$ & $U(1)_{R}$ \\
\hline$q$ & $\bar{f}$ & $\bar{f}$ & $2 r_{q}=\frac{3}{N_{f}}\left(1-\frac{1}{N_{f}-3}\right)$ \\
$q_{0}$ & $\bar{f}$ & 1 & $2 r_{q_{0}}=1-\frac{1}{N_{f}-3}$ \\
$s$ & $T_{S}$ & 1 & $2 r_{s}=\frac{2}{N_{f}-3}$ \\
$M$ & 1 & $T_{S}$ & $2 r_{M}=2-\frac{8}{N_{f}}$ \\
\hline
\end{tabular}

Corresponding superconformal indices are described by the integrals

$$
I_{E}=\frac{(p ; p)_{\infty}^{2}(q ; q)_{\infty}^{2}}{2^{2} 3} \prod_{m=1}^{N_{f}} \Gamma\left(t_{m} ; p, q\right) \int_{\mathbb{T}^{2}} \frac{\prod_{k=1}^{3} \prod_{m=1}^{N_{f}} \Gamma\left(t_{m} z_{k}^{ \pm 1} ; p, q\right)}{\prod_{1 \leq j<k \leq 3} \Gamma\left(z_{j}^{ \pm 1} z_{k}^{ \pm 1} ; p, q\right)} \prod_{k=1}^{2} \frac{d z_{k}}{2 \pi \mathrm{i} z_{k}}
$$

where $z_{1} z_{2} z_{3}=1$, and

$$
\begin{aligned}
& I_{M}=\frac{(p ; p)_{\infty}^{N_{f}-4}(q ; q)_{\infty}^{N_{f}-4}}{\left(N_{f}-3\right) !} \prod_{1 \leq j<k \leq N_{f}} \Gamma\left(t_{j} t_{k} ; p, q\right) \prod_{j=1}^{N_{f}} \Gamma\left(t_{j}^{2} ; p, q\right) \\
& \quad \times \int_{\mathbb{T}^{N_{f}-4}} \prod_{1 \leq j<k \leq N_{f}-3} \frac{\Gamma\left((p q)^{r_{s}} z_{j} z_{k} ; p, q\right)}{\Gamma\left(z_{j}^{-1} z_{k}, z_{j} z_{k}^{-1} ; p, q\right)} \prod_{j=1}^{N_{f}-3} \Gamma\left((p q)^{r_{s}} z_{j}^{2} ; p, q\right) \\
& \quad \times \prod_{j=1}^{N_{f}-3} \Gamma\left((p q)^{\left(1-r_{s}\right) / 2} z_{j}^{-1} ; p, q\right) \prod_{k=1}^{N_{f}} \Gamma\left((p q)^{\left(1-r_{s}\right) / 2} t_{k}^{-1} z_{j}^{-1} ; p, q\right) \prod_{j=1}^{N_{f}-4} \frac{d z_{j}}{2 \pi \mathrm{i} z_{j}}
\end{aligned}
$$

where $\prod_{j=1}^{N_{f}-3} z_{j}=1$, and the balancing condition reads $\prod_{m=1}^{N_{f}} t_{m}=(p q)^{\left(N_{f}-4\right) / 2}$. The equality $I_{E}=I_{M}$ represents a new symmetry transformation formula for general elliptic hypergeometric integrals on the $G_{2}$ root system. Independently, it was also considered earlier by F. A. Dolan.

For $N_{f}=5$ the integral $I_{M}$ takes the form

$$
\begin{aligned}
I_{M}= & \frac{(p ; p)_{\infty}(q ; q)_{\infty}}{2} \prod_{1 \leq j<k \leq 5} \Gamma\left(t_{j} t_{k} ; p, q\right) \prod_{j=1}^{5} \Gamma\left(t_{j}^{2} ; p, q\right) \\
& \times \int_{\mathbb{T}} \frac{\Gamma\left((p q)^{1 / 4} z_{j}^{ \pm 1} ; p, q\right) \prod_{k=1}^{5} \Gamma\left((p q)^{1 / 4} t_{k}^{-1} z_{j}^{ \pm 1} ; p, q\right)}{\Gamma\left(z^{ \pm 2} ; p, q\right)} \frac{d z}{2 \pi \mathrm{i} z} .
\end{aligned}
$$

Using the univariate elliptic beta integral, one can compute this $I_{M}$ and find the index coinciding with (13.2). As to the general $G_{2}$-transformation $I_{E}=I_{M}$, it should be a consequence of the original $S U(3)$-gauge group Seiberg duality. Indeed, let us take $N=3$ and set $t_{i}^{-1}=s_{i}$ in the electric index (4.6). Then, if we impose the constraint $s_{N_{f}}=p q$, we obtain the $G_{2}$-group electric index (13.3) with $N_{f}$ and $t_{i}$ replaced by $N_{f}-1$ and $s_{i}$, respectively. Therefore it is expected that the $G_{2}$-magnetic index can be obtained after appropriate restrictions on $I_{M}$ in (4.7). A difficulty lies in computing the limit $s_{N_{f}} \rightarrow p q$, since it leads to a diverging integral multiplied by a vanishing coefficient. This limit is currently under investigation.

\section{4. 'T HOOFT ANOMALY MATCHING CONDITIONS}

In this section we describe the standard 't Hooft anomaly matching conditions [40, 97] for some of the new dualities. Needed Casimir operators for unitary and symplectic groups can be found in Appendix C. There exist also the discrete anomalies matching conditions [14], but we skipped their consideration in the present work.

Multiple $S P(2 N)$ duality. Let us begin with the multiple duality for $S P(2 N)$ gauge group found in [91] and discussed in Sect. 6. Coincidence of the anomalies is checked for the smaller 
flavor groups of dual theories. The subgroup $S U(4) \times S U(4) \times U(1)_{B} \times U(1) \times U(1)_{R}$ of the electric theory has the following triangle anomaly coefficients:

$$
\begin{aligned}
& S U(4)_{L}^{3} \quad 2 N, \\
& S U(4)_{L}^{2} \times U(1) \quad \frac{3 N^{2}-2 N-1}{2}, \\
& U(1)_{B}^{2} \times U(1) \quad-4 N(N-1), \\
& S U(4)_{L}^{2} \times U(1)_{R} \quad-2 N^{2}+1 \\
& U(1)^{2} \times U(1)_{B} \quad 0, \\
& U(1)_{R} \quad-\left(2 N^{2}+7 N+1\right), \\
& S U(4)_{L}^{2} \times U(1)_{B} \quad 2 N \\
& U(1)_{B}^{2} \times U(1)_{R} \quad 0 \\
& U(1)^{2} \times U(1)_{R} \quad-\frac{N^{3}-1}{2} \\
& U(1)_{R}^{3} \\
& -\left(2 N^{2}+N+1\right) \text {. }
\end{aligned}
$$

We have verified that all three dual magnetic theories have the same anomaly coefficients. Also it is easy to check that the real anomaly is equal to zero in the electric and magnetic theories. Explicitly, for the electric theory one has: $2 N+2-\frac{1}{2} 8-(2 N-2)=0$.

$S P \leftrightarrow S P$ groups duality. Here we discuss the duality of Sect. 7. In the electric theory, anomaly coefficients for $S U(4) \times S P\left(2 l_{1}\right) \times S P\left(2 l_{2}\right) \times \ldots \times S P\left(2 l_{K}\right) \times U(1) \times U(1)_{R}$ global symmetry group have the values

$$
\begin{array}{rlll}
S U(4)^{3} & -2 M, & S U(4)^{2} \times U(1) & -\frac{1}{2} M(M-N-2) \\
S P\left(2 l_{i}\right)^{2} \times U(1) & -M n_{i}, & S U(4)^{2} \times U(1)_{R} & -2 M \\
S P\left(2 l_{i}\right)^{2} \times U(1)_{R} & 0, & U(1)_{R} & 1-6 M \\
U(1)_{R}^{3} & 1-6 M & & \\
U(1)^{2} \times U(1)_{R} & \frac{1}{2}\left(-M^{3}+2 N M^{2}-M N^{2}-4 M N-2 M+2\right)
\end{array}
$$

coinciding with the coefficients in the magnetic theory. Computation of the real gauge anomaly coefficient yields: $-4-(2 M-2)+2 M+2=0$.

Multiple $S U(2 N)$ duality. The electric theory of Sect. 8 has the following anomaly coefficients for $S U(4) \times S U(4) \times U(1)_{1} \times U(1)_{2} \times U(1)_{B} \times U(1)_{R}$ global symmetry group:

$$
\begin{array}{rlll}
S U(4)^{3} & 2 N, & S U(4)^{2} \times U(1)_{1} & 0 \\
S U(4)^{2} \times U(1)_{B} & 2 N, & S U(4)^{2} \times U(1)_{2} & 4 N(N-1) \\
S U(4)^{2} \times U(1)_{R} & -N, & U(1)_{1}^{2} \times U(1)_{B} & 0 \\
U(1)_{1}^{2} \times U(1)_{2} & -8 N(2 N-1), & U(1)_{1}^{2} \times U(1)_{R} & -2 N(2 N-1) \\
U(1)_{B}^{2} \times U(1)_{1} & 0, & U(1)_{B}^{2} \times U(1)_{2} & 32 N(2 N-1) \\
U(1)_{B}^{2} \times U(1)_{R} & -8 N, & U(1)_{2}^{2} \times U(1)_{1} & 0 \\
U(1)_{2}^{2} \times U(1)_{B} & 0, & U(1)_{2}^{2} \times U(1)_{R} & -32 N(N-1)^{2} \\
U(1)_{R} & -8 N+4 N^{2}-1, & U(1)_{R}^{3} & -2 N+4 N^{2}-1
\end{array}
$$

which coincide with the anomaly coefficients in all three dual magnetic theories. Calculation of the real gauge anomaly yields: $-\frac{1}{2} 8-2(2 N-2)+4 N=0$. 
New confining duality. The electric theory of Sect. 12.1.14 has the following anomaly coefficients for $S P(2 N) \times S U(N+3) \times U(1)_{1} \times U(1)_{2} \times U(1)_{R}$ global symmetry group

$$
\begin{array}{clll}
S U(N+3)^{3} & N+1, & U(1)_{R}^{3} & -\frac{1}{2}(N+2)(N+3) \\
S U(N+3)^{2} \times U(1)_{R} & -(N+1), & S P(2 N)^{2} \times U(1) & -\frac{(N+1)(N+3)}{2} \\
S U(N+3)^{2} \times U(1) & N+1, & \\
U(1)^{2} \times U(1)_{R} & -\frac{1}{2}(N+1)^{2}(N+2)(N+3) & \\
U(1)_{R} & -\frac{1}{2}(N+2)(N+3), & S P(2 N)^{2} \times U(1)_{R} & 0
\end{array}
$$

and the same picture holds for the magnetic partner. Calculation of the real gauge anomaly yields: $-(N+3)-(N-1)+2(N+1)=0$.

$S U \leftrightarrow S P$ groups duality. The anomaly matching for the common global group $S U(N+$ $3) \times S U(N+3) \times U(1)_{B} \times U(1)_{R}$ of the duality described in Sect. 11.1 is checked and yields:

$$
\begin{array}{rllr}
S U(N+3)_{L}^{3} & N+1, & S U(N+3)_{L}^{2} \times U(1)_{R} & -\frac{(N+1)^{2}}{N+3} \\
S U(N+3)_{L}^{2} \times U(1)_{B} & 2(N+1), & U(1)_{B}^{2} \times U(1)_{R} & -8(N+1)^{2} \\
U(1)_{R} & -\left(N^{2}+2 N+2\right), & U(1)_{R}^{3}-\frac{N^{4}-9 N^{2}-10 N+2}{(N+3)^{2}} .(14.5)
\end{array}
$$

$S U \leftrightarrow S U$ groups duality. Here we consider the dualities of Sect. 11.2. The anomaly matching is checked for the global group $S U(K)_{L} \times S U(N+3-K)_{L} \times U(1)_{1} \times S U(K)_{R} \times$ $S U(N+3-K)_{R} \times U(1)_{2} \times U(1)_{B} \times U(1)_{R}$ yielding

$$
\begin{array}{rlrl}
S U(K)_{L}^{3} & N+1, & S U(K)_{L}^{2} \times U(1)_{R} & -\frac{(N+1)^{2}}{N+3} \\
S U(K)_{L}^{2} \times U(1)_{B} & (N+1), & S U(K)_{L}^{2} \times U(1)_{1} & (N+1)(N+3-K) \\
U(1)_{B}^{2} \times U(1)_{1} & 0, & U(1)_{B}^{2} \times U(1)_{R} & -2(N+1)^{2} \\
U(1)_{1}^{2} \times U(1)_{B} & (N+1)(N+3) K(N+3-K) & \\
U(1)_{1}^{2} \times U(1)_{R} & -(N+1)^{2} K(N+3-K) & -\frac{N^{4}-9 N^{2}-10 N+2}{(N+3)^{2}} \\
U(1)_{R} & -\left(N^{2}+2 N+2\right) & U(1)_{R}^{3} & -\frac{14}{(N)}
\end{array}
$$

Computation of the real gauge anomaly yields: $-2(N+1)+2(N+1)=0$.

Comparing the 't Hooft anomaly matching conditions for all dualities described in this paper and the analysis of total ellipticity of the elliptic hypergeometric terms lying behind the equalities of superconformal indices, we come to the following conjecture.

Conjecture. The condition of total ellipticity for an elliptic hypergeometric term is necessary and sufficient for validity of the 't Hooft anomaly matching conditions for dual superconformal field theories whose superconformal indices are determined by this term.

For proving this hypothesis it is necessary to use the formal mathematical definition of anomalies as cocycles of the gauge groups (see, e.g., [71]). For dual theories we have two, in general different, gauge groups. Therefore the anomaly matching condition looks like an equality 
of Chern classes of dual theories, and the conditions of total ellipticity - as a condition of vanishing of the combined Chern classes. This problem deserves a separate detailed discussion.

\section{Conclusion}

To summarize our results, on the mathematical side we have conjectured many new symmetry transformations for elliptic hypergeometric integrals or exact evaluation formulas. On the physical side, we have found many new Seiberg dualities. Sections 6, 7, 8, and 11 contain new electric-magnetic dualities for $\mathcal{N}=1$ SYM theories based on unitary and symplectic gauge groups with specific matter content. Sections 12.1 .14 and 12.3 .3 contain new examples of $S$-confining theories derived from known identities for elliptic hypergeometric integrals.

It should be clear that this paper does not contain a description of all known dual superconformal field theories. We have limited ourselves only to simple gauge groups $G=S U(N), S P(2 N)$, and $G_{2}$. First, there are other simple groups $G=S O(N), F_{4}, E_{6}, E_{7}, E_{8}$ consideration of which we have skipped. The situation with the dualities for the exceptional groups [22, 48, 70] is not clear in general (except of the $G_{2}$-cases described above) due to the complexity of the invariants of these groups $[11,63]$. There are very many dualities involving orthogonal groups $S O(N)$. Originally we hoped to tackle them as well, but their amount is very big, and it was decided to consider them separately. It is known that many group-theoretical objects for the $S O(N)$ groups can be obtained as reductions of the $S P(2 N)$-group constructions. Some of such reductions were considered by Dolan and Osborn at the level of superconformal indices [26]. However, there are many dualities that they did not analyze. Many elliptic hypergeometric integrals for the $B_{N}$ (i.e., $S O(2 N+1)$ groups) and $D_{N}$ (i.e., $S O(2 N)$ groups) root systems can be obtained by special restriction of the $B C_{N}$-integrals (cf. the forms of the corresponding invariant measures given in Appendix B). However, it is not clear at the moment whether superconformal indices of all known $S O(N)$-group theories and their duals can be obtained in this way. There are also other types of reduction of the indices and dualities, e.g., those leading to dualities outside the conformal windows [92].

Second, we have deliberately skipped consideration of the superconformal indices for extended $\mathcal{N}>1$ supersymmetric field theories [6, 49]. The best known examples correspond to the Seiberg-Witten $\mathcal{N}=2$ theories $[77,78]$. Consider the following electric and magnetic theories

\begin{tabular}{|c|c|c|c|}
\hline & $S O(3)$ & $S U(3)$ & $U(1)_{R}$ \\
\hline$Q$ & $f$ & $f$ & $\frac{2}{3}$ \\
\hline
\end{tabular}

\begin{tabular}{|c|c|c|c|}
\hline & $S O(4)$ & $S U(3)$ & $U(1)_{R}$ \\
\hline$q$ & $f$ & $\bar{f}$ & $\frac{1}{3}$ \\
$M$ & 1 & $T_{S}$ & $\frac{4}{3}$ \\
\hline
\end{tabular}

As discussed by Intriligator and Seiberg [44, 45, 46] (see also [32]), the $S O(3)$ Seiberg duality electric model becomes the $S U(2)$ group $\mathcal{N}=4$ super-Yang-Mills theory in the infrared region after introducing the tree level superpotential $W_{\text {tree }} \propto \operatorname{det} Q$. Superconformal indices have the form

$$
I_{E}=\frac{(p ; p)_{\infty}(q ; q)_{\infty}}{2} \prod_{j=1}^{3} \Gamma\left((p q)^{1 / 3} s_{j} ; p, q\right) \int_{\mathbb{T}} \frac{\prod_{j=1}^{3} \Gamma\left((p q)^{1 / 3} s_{j} z^{ \pm 1} ; p, q\right)}{\Gamma\left(z^{ \pm 1} ; p, q\right)} \frac{d z}{2 \pi \mathrm{i} z}
$$

where $\prod_{j=1}^{3} s_{j}=1$, and

$$
\begin{aligned}
I_{M}= & \frac{(p ; p)_{\infty}^{2}(q ; q)_{\infty}^{2}}{4} \prod_{1 \leq i<j \leq 3} \Gamma\left((p q)^{\frac{2}{3}} s_{i} s_{j} ; p, q\right) \prod_{i=1}^{3} \Gamma\left((p q)^{\frac{2}{3}} s_{i}^{2} ; p, q\right) \\
& \times \int_{\mathbb{T}^{2}} \frac{\prod_{j=1}^{2} \prod_{i=1}^{3} \Gamma\left((p q)^{1 / 6} s_{i}^{-1} z_{j}^{ \pm 1} ; p, q\right)}{\Gamma\left(z_{1}^{ \pm 1} z_{2}^{ \pm 1} ; p, q\right)} \prod_{j=1}^{2} \frac{d z_{j}}{2 \pi \mathrm{i} z_{j}} .
\end{aligned}
$$


By a change of integration variables $y_{1}=\sqrt{z_{1} z_{2}}, y_{2}=\sqrt{z_{1} / z_{2}}$ in $I_{M}$, one of the integrations can be taken explicitly with the help of univariate elliptic beta integral, which shows that (15.2) is equal to (15.1). This equality can be obtained as a reduction of the $B C_{N}$-relations as well [26]. We suppose therefore that it is necessary to consider first all possible $S P(2 N)$-group identities for integrals and then try to reduce them to the relations for superconformal indices of extended supersymmetric dual theories with $S O(N)$ gauge groups.

Third, we skipped the quiver gauge group cases, when there is more than one simple gauge group (which corresponds also to the deconfinement phenomenon [5]). Is is expected that equalities of the superconformal indices for them are mere consequences of the so-called Bailey-type chains (forming a tree) of symmetry transformations for integrals discovered by the first author in [84] and extended in [93] to root systems. Within this context, the duality transformation acquires a simple meaning of the integral transform whose properties resemble the classical Fourier transformation, see [93].

Let us list some other possible applications of our results. Counting of the gauge invariant operators for a number of supersymmetric gauge theories was considered in detail in [34, 39]. It is not difficult to see that the corresponding generating functions are obtained from our superconformal indices by taking the limits $p, q \rightarrow 0$. To take the simplest possible limit $p \rightarrow 0$ one needs first to get rid of the balancing conditions by multiplying a number of parameters by integer powers of $p$ and applying the reflection formula for the elliptic gamma function, see [89]. However, in the present work we have a much larger list of theories where this gauge invariant operators counting technique is applicable (in particular, this concerns the theories described in Sects. 7, 8, 9.2-9.6, 10.2-10.4, 11-13). The limit $p \rightarrow 0$ in all these theories leads to $q$-hypergeometric functions, the meaning of which is not clarified yet from the superconformal index point of view. The subsequent limit $q \rightarrow 0$ can be replaced by $q \rightarrow 1$ yielding the plain hypergeometric functions, which also should have thus some topological meaning in gauge field theories. Similar clarification is needed for the situations when the elliptic hypergeometric integrals are reduced to terminating elliptic hypergeometric series by some special choices of the parameters, or for the relations between integrals with different powers of $p$ and $q$.

In $[83,88]$, the first author has constructed univariate biorthognal functions associated with the elliptic beta integral. Naturally, it was conjectured there that some multivariable biorthogonal functions exist for all known elliptic beta integrals (which serve as the orthogonality measures). The first family of such functions was constructed by Rains in [65, 66]. As a consequence of our work, the expected number of similar families of multivariable biorthogonal functions has now increased essentially.

In $[85,87]$, it was shown that some of the $B C_{N}$ elliptic hypergeometric integrals can be associated with the relativistic Calogero-Sutherland type models. It was conjectured there that other models of such type can be built out of all other existing elliptic beta integrals and their appropriate generalizations. Because we have now the interpretation of the elliptic hypergeometric integrals as superconformal indices of supersymmetric field theories, we come to the natural conjecture that behind each $\mathcal{N}=1$ superconformal field theory there is a CalogeroSutherland type model for which these integrals serve either as the topological indices or the wave functions normalizations, respectively. We would like to mention in this context the known appearance of the usual elliptic Calogero-Sutherland models within the $\mathcal{N}=2$ Seiberg-Witten theories [59].

The group-theoretical interpretation of the elliptic hypergeometric integrals discussed in [73, $26,91]$ and the present paper opens possibilities for general structural theorems on the integrals themselves. It may play a key role in the classification of such integrals on root systems. In particular, it naturally leads to the conjecture that there exist infinitely (countably) many 
dualities and related elliptic hypergeometric integral identities. All the problems mentioned above deserve detailed investigation either in relation to supersymmetric dualities or on plain mathematical grounds. As to the proofs of many new hypergeometric identities conjectured in this paper, we refer to known methods described in [19, 20, 65, 69, 81, 83, 84, 93] (or indicated above in some cases) which are available for their treatment. We plan to consider them case by case depending on their tractability.

Acknowledgments. We would like to thank A. A. Belavin, F. A. Dolan, D. I. Kazakov, A. Khmelnitsky, H. Osborn, A. F. Oskin, V. A. Rubakov, A. Schwimmer, M. A. Shifman and S. Theisen for valuable discussions, comments and remarks. The first author is indebted to L. D. Faddeev for discussions on elliptic hypergeometric functions and their applications, which inspired the choice of present paper's title (it matches in spirit with [98], and it is expected that there exists a noncommutative extension of the elliptic hypergeometry, formulation of which is a rather difficult task). He is also grateful to A. M. Vershik for persistent support and encouragement, which was the main driving force for writing the survey [89]. The second author would like to thank BLTP JINR for its creative atmosphere due to which he was able to join this project.

The first author was partially supported by RFBR grants no. 09-01-00271, 09-01-93107NCNIL-a and, at the final stages of work, by CERN TH (Geneva) and MPIM (Bonn). The second author was partially supported by the Dynasty foundation, RFBR grant no. 08-0200856-a and grant of the Ministry of Education and Science of the Russian Federation no. 1027.2008.2.

\section{Appendix A. Characters of Representations of Classical groups}

Here we present general results for characters of the Lie group representations used in the paper. For the $S U(N)$ group representations, the characters, depending on $x=\left(x_{1}, \ldots, x_{N}\right)$ subject to the constraint $\prod_{i=1}^{N} x_{i}=1$, are the well known Schur polynomials

$$
s_{\underline{\lambda}}(x)=s_{\left(\lambda_{1}, \ldots, \lambda_{N}\right)}(x)=\frac{\operatorname{det}\left[x_{i}^{\lambda_{j}+N-j}\right]}{\operatorname{det}\left[x_{i}^{N-j}\right]},
$$

where $\underline{\lambda}$ is the partition ordered so that $\lambda_{1} \geq \lambda_{2} \geq \ldots \geq \lambda_{N}$. They obey the property $s_{\left(\lambda_{1}, \ldots, \lambda_{N}\right)}(x)=s_{\left(\lambda_{1}+c, \ldots, \lambda_{N}+c\right)}(x)$, where $c \in \mathbb{Z}$. Therefore one can assume that $\lambda_{N}=0$ without loss of generality.

Let us list explicitly the simplest characters. The fundamental and antifundamental representations:

$$
\chi_{S U(N), f}(x)=s_{(1,0, \ldots, 0)}(x)=\sum_{i=1}^{N} x_{i}, \quad \chi_{S U(N), \bar{f}}=s_{(1, \ldots, 1,0)}(x)=\chi_{S U(N), f}\left(x^{-1}\right) .
$$

The adjoint representation:

$$
\chi_{S U(N), a d j}(x)=s_{(2,1, \ldots, 1,0)}(x)=\sum_{1 \leq i, j \leq N} x_{i} x_{j}^{-1}-1 .
$$

The absolutely anti-symmetric tensor representation of rank two:

$$
\chi_{S U(N), T_{A}}(x)=s_{(1,1,0, \ldots, 0)}(x)=\sum_{1 \leq i<j \leq N} x_{i} x_{j}, \quad \chi_{S U(N), \bar{T}_{A}}=\chi_{S U(N), T_{A}}\left(x^{-1}\right) .
$$


The symmetric representation:

$$
\chi_{S U(N), T_{S}}(x)=s_{(2,0, \ldots, 0)}(x)=\sum_{1 \leq i<j \leq N} x_{i} x_{j}+\sum_{i=1}^{N} x_{i}^{2}, \quad \chi_{S U(N), \bar{T}_{S}}(x)=\chi_{S U(N), T_{S}}\left(x^{-1}\right) .
$$

The absolutely anti-symmetric tensor representation of rank three:

$$
\chi_{S U(N), T_{3 A}}(x)=s_{(1,1,1,0, \ldots, 0)}(x)=\sum_{1 \leq i<j<k \leq N} x_{i} x_{j} x_{k} .
$$

The absolutely symmetric tensor representation of rank three:

$$
\chi_{S U(N), T_{3 S}}(x)=s_{(3,0, \ldots, 0)}(x)=\sum_{1 \leq i<j<k \leq N} x_{i} x_{j} x_{k}+\sum_{i, j=1, i \neq j}^{N} x_{i}^{2} x_{j}+\sum_{i=1}^{N} x_{i}^{3} .
$$

In the mixed case, we have

$$
\chi_{S U(N), T_{A S}}(x)=s_{(2,1,0, \ldots, 0)}(x)=2 \sum_{1 \leq i<j<k \leq N} x_{i} x_{j} x_{k}+\sum_{i, j=1 ; i \neq j}^{N} x_{i}^{2} x_{j} .
$$

The Weyl characters for $S P(2 N)$ group are given by the determinant

$$
\widetilde{s}_{\left(\lambda_{1}, \ldots, \lambda_{N}\right)}(x)=\frac{\operatorname{det}\left[x_{i}^{\lambda_{j}+N-j+1}-x_{i}^{-\lambda_{j}-N+j-1}\right]}{\operatorname{det}\left[x_{i}^{N-j+1}-x_{i}^{-N+j-1}\right]},
$$

with $\lambda_{1} \geq \lambda_{2} \geq \ldots \geq \lambda_{N} \geq 0$. For the fundamental and antifundamental representations

$$
\chi_{S P(2 N), f}(x)=\chi_{S P(2 N), \bar{f}}(x)=\widetilde{s}_{(1,0, \ldots, 0)}(x)=\sum_{i=1}^{N}\left(x_{i}+x_{i}^{-1}\right),
$$

For the adjoint representation

$$
\begin{aligned}
& \chi_{S P(2 N), a d j}(x)=\widetilde{s}_{(2,0, \ldots, 0)}(x) \\
& \quad=\sum_{1 \leq i<j \leq N}\left(x_{i} x_{j}+x_{i} x_{j}^{-1}+x_{i}^{-1} x_{j}+x_{i}^{-1} x_{j}^{-1}\right)+\sum_{i=1}^{N}\left(x_{i}^{2}+x_{i}^{-2}\right)+N .
\end{aligned}
$$

For the absolutely anti-symmetric representation

$$
\chi_{S P(2 N), T_{A}}(x)=\widetilde{s}_{(1,1,0, \ldots, 0)}(x)=\sum_{1 \leq i<j \leq N}\left(x_{i} x_{j}+x_{i} x_{j}^{-1}+x_{i}^{-1} x_{j}+x_{i}^{-1} x_{j}^{-1}\right)+N-1 .
$$

As to the exceptional group $G_{2}$, its fundamental representation has the character

$$
\chi\left(z_{1}, z_{2}, z_{3}\right)=1+\sum_{i=1}^{3}\left(z_{i}+z_{i}^{-1}\right),
$$

where $z_{1} z_{2} z_{3}=1$. The character for the adjoint representation of $G_{2}$ group is

$$
\chi\left(z_{1}, z_{2}, z_{3}\right)=2+\sum_{1 \leq i<j \leq 3}\left(z_{i} z_{j}+z_{i}^{-1} z_{j}+z_{i} z_{j}^{-1}+z_{i}^{-1} z_{j}^{-1}\right) .
$$




\section{APPENDix B. INVARIANT MATRIX GROUP MEASURES}

Here we describe the invariant measures for integrals over classical Lie groups and the exceptional group $G_{2}$. Such a measure for the unitary group $S U(N)$ with any symmetric function $f(z)$, where $z=\left(z_{1}, \ldots, z_{N}\right), \prod_{j=1}^{N} z_{j}=1$, has the form

$$
\int_{S U(N)} f(z) d \mu(z)=\frac{1}{N !} \int_{\mathbb{T}^{N-1}} \Delta(z) \Delta\left(z^{-1}\right) f(z) \prod_{j=1}^{N-1} \frac{d z_{j}}{2 \pi \mathrm{i} z_{j}}
$$

where $\Delta(z)$ is the Vandermonde determinant

$$
\Delta(z)=\prod_{1 \leq i<j \leq N}\left(z_{i}-z_{j}\right)
$$

The invariant measure for the symplectic group $S P(2 N)$ with any symmetric function $f(z), z=$ $\left(z_{1}, \ldots, z_{N}\right)$, has the form

$$
\int_{S P(2 N)} f(z) d \mu(z)=\frac{(-1)^{N}}{2^{N} N !} \int_{\mathbb{T}^{N}} \prod_{j=1}^{N}\left(z_{j}-z_{j}^{-1}\right)^{2} \Delta\left(z+z^{-1}\right)^{2} f(z) \prod_{j=1}^{N} \frac{d z_{j}}{2 \pi \mathrm{i} z_{j}}
$$

For the invariant measures over the orthogonal group $S O(N)$ and any symmetric function $f(z), z=\left(z_{1}, \ldots, z_{N}\right)$, one has to distinguish the cases of odd and even $N$ :

$$
\int_{S O(2 N)} f(z) d \mu(z)=\frac{1}{2^{N-1} N !} \int_{\mathbb{T}^{N}} \Delta\left(z+z^{-1}\right)^{2} f(z) \prod_{j=1}^{N} \frac{d z_{j}}{2 \pi \mathrm{i} z_{j}}
$$

and

$$
\int_{S O(2 N+1)} f(z) d \mu(z)=\frac{(-1)^{N}}{2^{N} N !} \int_{\mathbb{T}^{N}} \prod_{j=1}^{N}\left(z_{j}^{\frac{1}{2}}-z_{j}^{-\frac{1}{2}}\right)^{2} \Delta\left(z+z^{-1}\right)^{2} f(z) \prod_{j=1}^{N} \frac{d z_{j}}{2 \pi \mathrm{i} z_{j}}
$$

The invariant measure for the exceptional group $G_{2}$ and any symmetric function $f(z), z=$ $\left(z_{1}, z_{2}, z_{3}\right)$, where $z_{1} z_{2} z_{3}=1$, has the form

$$
\int_{G_{2}} f(z) d \mu(z)=\frac{1}{2^{2} 3} \int_{\mathbb{T}^{2}} \Delta\left(z+z^{-1}\right)^{2} f(z) \prod_{j=1}^{2} \frac{d z_{j}}{2 \pi \mathrm{i} z_{j}} .
$$

\section{Appendix C. Relevant Casimir operators}

Commutators of the generators $T^{a}, a=1, \ldots, \operatorname{dim} G$, of some classical Lie group $G$ are defined with the help of structure constants $f^{a b c}$

$$
\left[T^{a}, T^{b}\right]=i f^{a b c} T^{c} .
$$

It is straightforward to obtain the Casimir operators [97]

$$
\sum_{a, l}\left(T_{\mathbf{r}}^{a}\right)_{l}^{m}\left(T_{\mathbf{r}}^{a}\right)_{n}^{l}=C_{2}(\mathbf{r}) \delta_{n}^{m}, \quad \sum_{n, m}\left(T_{\mathbf{r}}^{a}\right)_{n}^{m}\left(T_{\mathbf{r}}^{b}\right)_{m}^{n}=T(\mathbf{r}) \delta^{a b}
$$

where $\mathbf{r}$ is some irreducible representation. These Casimir operators and the dimension of the representation $d(\mathbf{r})$ are connected through the adjoint representation adj,

$$
d(\mathbf{r}) C_{2}(\mathbf{r})=d(\mathbf{a d j}) T(\mathbf{r})
$$

For checking the 't Hooft anomaly matching conditions we need the triple Casimir operator which comes from the trace

$$
A^{a b c}=\operatorname{Tr}\left[T^{a}\left\{T^{b}, T^{c}\right\}\right] .
$$


Then it is convenient to define the operator $A(\mathbf{r})$ relative to the fundamental representation

$$
A^{a b c}(\mathbf{r})=A(\mathbf{r}) \mathbf{A}^{a b c}
$$

where $\mathbf{A}^{a b c}=\operatorname{Tr}\left[T_{F}^{a}\left\{T_{F}^{b}, T_{F}^{c}\right\}\right]$ and $T_{F}^{a}$ are the generators in the fundamental representation.

In the tables below we give the dimensions $d(\mathbf{r})$, the Casimir operators $2 T(\mathbf{r})$, and $A(\mathbf{r})$ for the unitary group and the dimensions $d(\mathbf{r})$, the Casimir operators $T(\mathbf{r})$ for symplectic and $G_{2}$ groups. Note that in the verification of the anomaly matchings for unitary groups we use $2 T(\mathbf{r})$.

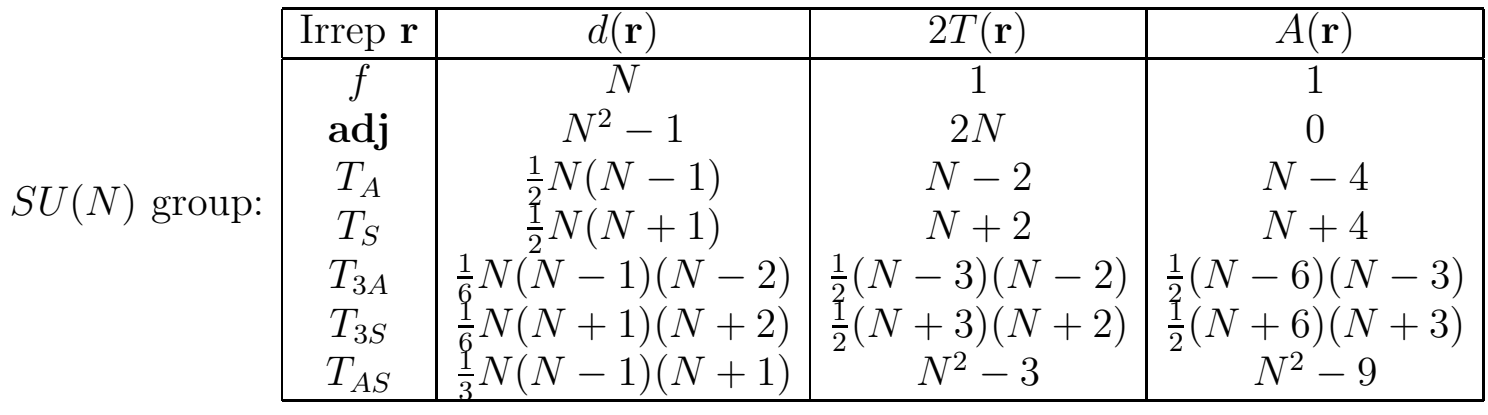

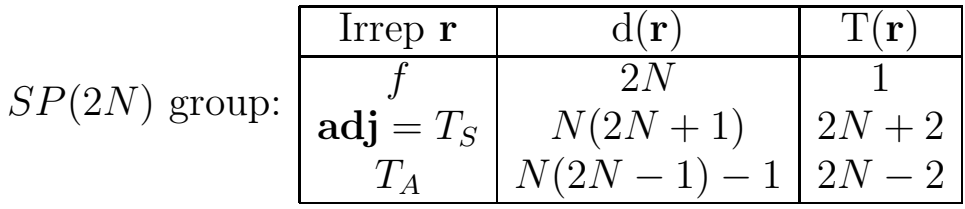

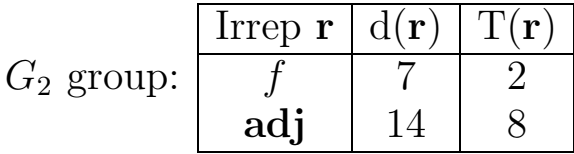

\section{APPENDix D. TOtal ELLIPTICITY FOR THE KS DUALity INDICES}

In order to illustrate the work hidden behind our conjectures, we briefly describe in this Appendix verification of the total ellipticity for the transformation identity for elliptic hypergeometric integrals associated with the Kutasov-Schwimmer duality from Sect. 9.1.

First, we change the integration variables $\underline{z}$ in $(9.3)$ to $\underline{z}=U^{-1}(S T)^{-\frac{K}{2 N}} \underline{y}$ and assume that the contours of integration in $y$-variables can be deformed back to $\mathbb{T}$ without crossing the poles. Then the equality of integrals (9.2) and (9.3) is rewritten in the following form

$$
\kappa_{N} \int_{\mathbb{T}^{N-1}} \Delta_{E}(\underline{z}, \underline{t}, \underline{s}) \prod_{j=1}^{N-1} \frac{d z_{j}}{2 \pi \mathrm{i} z_{j}}=\kappa_{\widetilde{N}} \int_{\mathbb{T}^{\widetilde{N}-1}} \Delta_{M}(\underline{y}, \underline{t}, \underline{s}) \prod_{j=1}^{\widetilde{N}-1} \frac{d y_{j}}{2 \pi \mathrm{i} y_{j}}
$$

where $\tilde{N}=K N_{f}-N$ and

$$
\kappa_{N}=\frac{(p ; p)_{\infty}^{N-1}(q ; q)_{\infty}^{N-1}}{N !} \Gamma(U ; p, q)^{N-1}
$$


with $U=(p q)^{\frac{1}{K+1}}$. The kernels of the elliptic hypergeometric integrals are

$$
\begin{aligned}
\Delta_{E}(\underline{z}, \underline{t}, \underline{s})= & \prod_{1 \leq i<j \leq N} \frac{\Gamma\left(U z_{i} z_{j}^{-1}, U z_{i}^{-1} z_{j} ; p, q\right)}{\Gamma\left(z_{i} z_{j}^{-1}, z_{i}^{-1} z_{j} ; p, q\right)} \prod_{i=1}^{N_{f}} \prod_{j=1}^{N} \Gamma\left(s_{i} z_{j}, t_{i}^{-1} z_{j}^{-1} ; p, q\right), \\
\Delta_{M}(\underline{y}, \underline{t}, \underline{s})= & \prod_{l=1}^{K} \prod_{i, j=1}^{N_{f}} \Gamma\left(U^{l-1} s_{i} t_{j}^{-1} ; p, q\right) \prod_{1 \leq i<j \leq \tilde{N}} \frac{\Gamma\left(U y_{i} y_{j}^{-1}, U y_{i}^{-1} y_{j} ; p, q\right)}{\Gamma\left(y_{i} y_{j}^{-1}, y_{i}^{-1} y_{j} ; p, q\right)} \\
& \times \prod_{i=1}^{N_{f}} \prod_{j=1}^{\widetilde{N}} \Gamma\left(s_{i}^{-1} y_{j}, U^{2} t_{i} y_{j}^{-1} ; p, q\right)
\end{aligned}
$$

where $\prod_{i=1}^{N} z_{i}=1, \prod_{i=1}^{\widetilde{N}} y_{i}=U^{\widetilde{N}+N K}(p q)^{-\frac{1}{2} N_{f} K} S^{K}$, and $U^{2 N} S T^{-1}=(p q)^{N_{f}}$.

Theorem 5. The function

$$
\rho(\underline{z}, \underline{y}, \underline{t}, \underline{s})=\frac{\Delta_{E}(\underline{z}, \underline{t}, \underline{s})}{\Delta_{M}(\underline{y}, \underline{t}, \underline{s})}
$$

is a totally elliptic hypergeometric term.

Ellipticity of the $z$-variables $q$-certificates. As described in Sect. 2, we should consider the ratios

$$
\begin{aligned}
h_{a}^{z}(\underline{z}, \underline{y}, \underline{t}, \underline{s}, q) & =\frac{\left.\rho(\underline{z}, \underline{y}, \underline{t}, \underline{s})\right|_{z_{a} \rightarrow q z_{a}, z_{N} \rightarrow q^{-1} z_{N}}}{\rho(\underline{z}, \underline{y}, \underline{t}, \underline{s})} \\
= & \prod_{j=1, j \neq a}^{N-1} \frac{\theta\left(U z_{a} z_{j}^{-1}, U z_{j} z_{N}^{-1}, q^{-1} z_{a}^{-1} z_{j}, q^{-1} z_{j}^{-1} z_{N} ; p\right)}{\theta\left(U q^{-1} z_{a}^{-1} z_{j}, U q^{-1} z_{j}^{-1} z_{N}, z_{a} z_{j}^{-1}, z_{j} z_{N}^{-1} ; p\right)} \\
& \times \frac{\theta\left(U q z_{a} z_{N}^{-1}, U z_{a} z_{N}^{-1}, q^{-2} z_{a}^{-1} z_{N}, q^{-1} z_{a}^{-1} z_{N} ; p\right)}{\theta\left(U q^{-1} z_{a}^{-1} z_{N}, U q^{-2} z_{a}^{-1} z_{N}, q z_{a} z_{N}^{-1}, z_{a} z_{N}^{-1} ; p\right)} \prod_{i=1}^{N_{f}} \frac{\theta\left(s_{i} z_{a} ; p\right)}{\theta\left(q^{-1} s_{i} z_{N} ; p\right)} \frac{\theta\left(t_{i}^{-1} z_{N}^{-1} ; p\right)}{\theta\left(q^{-1} t_{i}^{-1} z_{a}^{-1} ; p\right)},
\end{aligned}
$$

where $a=1, \ldots, N-1$, and check that these are totally elliptic functions. Indeed, $h_{a}^{z}(\underline{z}, \underline{y}, \underline{t}, \underline{s}, q)$ functions are automatically invariant under the transformations 1) $s_{b} \rightarrow p s_{b}, s_{N_{f}} \rightarrow p^{-1} s_{N_{f}}, 2$ ) $\left.t_{b} \rightarrow p t_{b}, t_{N_{f}} \rightarrow p^{-1} t_{N_{f}}, 3\right) y_{b} \rightarrow p y_{b}, y_{\widetilde{N}} \rightarrow p^{-1} y_{\widetilde{N}}$. Whereas the invariance with respect to the substitutions 4$) z_{c} \rightarrow p z_{c}, z_{N} \rightarrow p^{-1} z_{N}$ for $c \neq a$ or 5) $z_{a} \rightarrow p z_{a}, z_{N} \rightarrow p^{-1} z_{N}$ uses the balancing condition. Similarly, one checks the invariance with respect to the mixed transformations $s_{b} \rightarrow p s_{b}, t_{c} \rightarrow p t_{c}$ and $y_{d} \rightarrow p^{K} y_{d}$.

The most complicated part of the work consists in establishing ellipticity in the variable $q$. The difficulty comes from the presence of fractional powers of $q$ entering (D.3) through the variable $U$. Because of that one should scale $q$ by such a power of $p$ that there will be a match with the period of the elliptic functions $h_{a}^{z}(\underline{z}, \underline{y}, \underline{t}, \underline{s}, q)$. Simultaneously, we should preserve the balancing condition and all other constraints on the parameters we have. This is reached by the following transformation of the parameters

$$
\text { 6) } q \rightarrow p^{K+1} q, \quad t_{N_{f}}^{-1} \rightarrow p^{(K+1) N_{f}-2 N} t_{N_{f}}^{-1}, \quad y_{\widetilde{N}} \rightarrow p^{\widetilde{N}+N K-N_{f} K(K+1) / 2} y_{\widetilde{N}}
$$

which leads to $U \rightarrow p U$, as required. It is a matter of a neat computation (at the intermediate steps there appears a very cumbersome expression) to show that $h_{a}^{z}(\underline{z}, \underline{y}, \underline{t}, \underline{s}, q)$ do not change under these substitutions. 
Ellipticity of the $y$-variables $q$-certificates. Now we consider the ratios

$$
\begin{aligned}
h_{a}^{y}(\underline{z}, \underline{y}, \underline{t}, \underline{s}, q) & =\frac{\left.\rho(\underline{z}, \underline{y}, \underline{t}, \underline{s})\right|_{y_{a} \rightarrow q y_{a}, y_{\widetilde{N}} \rightarrow q^{-1} y_{\widetilde{N}}}}{\rho(\underline{z}, \underline{y}, \underline{t}, \underline{s})} \\
= & \prod_{j=1, j \neq a}^{\widetilde{N}-1} \frac{\theta\left(U q^{-1} y_{a}^{-1} y_{j}, U q^{-1} y_{j}^{-1} y_{\widetilde{N}}, y_{a} y_{j}^{-1}, y_{j} y_{\widetilde{N}}^{-1} ; p\right)}{\theta\left(U y_{a} y_{j}^{-1}, U y_{j} y_{\widetilde{N}}^{-1}, q^{-1} y_{a}^{-1} y_{j}, q^{-1} y_{j}^{-1} y_{\widetilde{N}} ; p\right)} \\
& \times \frac{\theta\left(U q^{-1} y_{a}^{-1} y_{\widetilde{N}}, U q^{-2} y_{a}^{-1} y_{\widetilde{N}}, q y_{a} y_{\widetilde{N}}^{-1}, y_{a} y_{\widetilde{N}}^{-1} ; p\right)}{\theta\left(U q y_{a} y_{\widetilde{N}}^{-1}, U y_{a} y_{\widetilde{N}}^{-1}, q^{-2} y_{a}^{-1} y_{\widetilde{N}}, q^{-1} y_{a}^{-1} y_{\widetilde{N}} ; p\right)} \prod_{i=1}^{N_{f}} \frac{\theta\left(q^{-1} s_{i}^{-1} y_{\widetilde{N}} ; p\right)}{\theta\left(s_{i}^{-1} y_{a} ; p\right)} \frac{\theta\left(U^{2} q^{-1} t_{i} y_{a}^{-1} ; p\right)}{\theta\left(U^{2} t_{i} y_{\widetilde{N}}^{-1} ; p\right)},
\end{aligned}
$$

where $a=1, \ldots, \widetilde{N}-1$. Again, these are the totally elliptic functions. They are automatically invariant under the transformations 1) $\left.\left.s_{b} \rightarrow p s_{b}, s_{N_{f}} \rightarrow p^{-1} s_{N_{f}}, 2\right) t_{b} \rightarrow p t_{b}, t_{N_{f}} \rightarrow p^{-1} t_{N_{f}}, 3\right)$ $z_{b} \rightarrow p z_{b}, z_{N} \rightarrow p^{-1} z_{N}$. The invariance with respect to the substitutions 4) $y_{b} \rightarrow p y_{b}, y_{\widetilde{N}} \rightarrow$ $p^{-1} y_{\widetilde{N}}, b \neq a$, or 5) $y_{a} \rightarrow p y_{a}, y_{\widetilde{N}} \rightarrow p^{-1} y_{\widetilde{N}}$ uses the balancing condition. The most difficult part is the verification of the invariance with respect to the transformations

$$
\text { 6) } q \rightarrow p^{K+1} q, \quad U \rightarrow p U, \quad t_{N_{f}}^{-1} \rightarrow p^{(K+1) N_{f}-2 N} t_{N_{f}}^{-1}, \quad y_{\widetilde{N}} \rightarrow p^{\widetilde{N}+N K-N_{f} K(K+1) / 2} y_{\widetilde{N}} .
$$

Ellipticity of the $t$-parameters $q$-certificates. Now we need to investigate the functions

$$
\begin{aligned}
h_{a}^{t}(\underline{z}, \underline{y}, \underline{t}, \underline{s}, q) & =\frac{\left.\rho(\underline{z}, \underline{y}, \underline{t}, \underline{s})\right|_{t_{a} \rightarrow q t_{a}, t_{N_{f}} \rightarrow q^{-1} t_{N_{f}}}}{\rho(\underline{z}, \underline{y}, \underline{t}, \underline{s})} \\
\quad= & \prod_{l=1}^{K} \prod_{i=1}^{N_{f}} \frac{\theta\left(U^{l-1} q^{-1} s_{i} t_{a}^{-1} ; p\right)}{\theta\left(U^{l-1} s_{i} t_{N_{f}}^{-1} ; p\right)} \prod_{j=1}^{N} \frac{\theta\left(t_{N_{f}}^{-1} z_{j}^{-1} ; p\right)}{\theta\left(q^{-1} t_{a}^{-1} z_{j}^{-1} ; p\right)} \prod_{i=1}^{\widetilde{N}} \frac{\theta\left(U^{2} q^{-1} t_{N_{f}} y_{j}^{-1} ; p\right)}{\theta\left(U^{2} t_{a} y_{j}^{-1} ; p\right)},
\end{aligned}
$$

where $a=1, \ldots, N_{f}-1$, and show that they are totally elliptic. Again, invariance under 1) $y_{b} \rightarrow$ $\left.p y_{b}, y_{\widetilde{N}} \rightarrow p^{-1} y_{\widetilde{N}}, 2\right) s_{b} \rightarrow p s_{b}, s_{N_{f}} \rightarrow p^{-1} s_{N_{f}}$, and 3) $z_{b} \rightarrow p z_{b}, z_{N} \rightarrow p^{-1} z_{N}$ transformations is automatic. The balancing condition is needed for symmetries 4$) t_{c} \rightarrow p t_{c}, t_{N_{f}} \rightarrow p^{-1} t_{N_{f}}, c \neq a$, and 5) $t_{a} \rightarrow p t_{a}, t_{N_{f}} \rightarrow p^{-1} t_{N_{f}}$ Computations during the verification of invariance under the transformations

6) $q \rightarrow p^{K+1} q, \quad U \rightarrow p U, \quad t_{N_{f}-1}^{-1} \rightarrow p^{(K+1) N_{f}-2 N} t_{N_{f}-1}^{-1}, \quad y_{\widetilde{N}} \rightarrow p^{\widetilde{N}+N K-N_{f} K(K+1) / 2} y_{\widetilde{N}}$

are very lengthy and require a lot of attention to reach the needed statement. Consideration of the $s$-parameters certificates is equivalent to the $t$-variables case because of the symmetries of the initial integral kernels.

\section{REFERENCES}

[1] G. E. Andrews, R. Askey, and R. Roy, Special Functions, Encyclopedia of Math. Appl. 71, Cambridge Univ. Press, Cambridge, 1999.

[2] E. W. Barnes, On the theory of the multiple gamma function, Cambr. Trans. 19 (1904), 374-425.

[3] R. J. Baxter, Partition function of the eight-vertex lattice model, Ann. Phys. (NY) 70 (1972), 193-228.

[4] S. Benvenuti, B. Feng, A. Hanany and Y. H. He, Counting BPS Operators in Gauge Theories: Quivers, Syzygies and Plethystics, JHEP 0711 (2007) 050, hep-th/0608050.

[5] M. Berkooz, The dual of supersymmetric $S U(2 k)$ with an antisymmetric tensor and composite dualities, Nucl. Phys. B452 (1995), 513-525, hep-th/9505067.

[6] M. Bianchi, F. A. Dolan, P. J. Heslop and H. Osborn, $\mathcal{N}=4$ superconformal characters and partition functions, Nucl. Phys. B767 (2007), 163-226, hep-th/0609179.

[7] J. H. Brodie, Duality in supersymmetric $S U\left(N_{c}\right)$ gauge theory with two adjoint chiral superfields, Nucl. Phys. B478 (1996), 123-140, hep-th/9605232. 
[8] J. H. Brodie and M. J. Strassler, Patterns of duality in $\mathcal{N}=1$ SUSY gauge theories, or: Seating preferences of theater going nonAbelian dualities, Nucl. Phys. B524 (1998), 224-250, hep-th/9611197.

[9] F. J. van de Bult, An elliptic hypergeometric beta integral transformation, arXiv:0912.3812 [math.CA].

[10] F. J. van de Bult and E. M. Rains, Basic hypergeometric functions as limits of elliptic hypergeometric functions, SIGMA 5 (2009), 59, 31 pp., arXiv:0902.0621 [math.CA].

[11] P. L. Cho, Moduli in exceptional SUSY gauge theories, Phys. Rev. D 57 (1998), 5214-5223, hep-th/9712116.

[12] P. L. Cho and P. Kraus, Symplectic SUSY gauge theories with antisymmetric matter, Phys. Rev. D54 (1996), 7640-7649, hep-th/9607200.

[13] C. Csáki and H. Murayama, Discrete anomaly matching, Nucl. Phys. B515 (1998), 114-162, hepth/9710105.

[14] C. Csáki and H. Murayama, New confining $\mathcal{N}=1$ supersymmetric gauge theories, Phys. Rev. D59 (1999) 065001, hep-th/9810014.

[15] C. Csáki, M. Schmaltz and W. Skiba, A systematic approach to confinement in $\mathcal{N}=1$ supersymmetric gauge theories, Phys. Rev. Lett. 78 (1997), 799-802, hep-th/9610139.

[16] C. Csáki, M. Schmaltz and W. Skiba, Confinement in $\mathcal{N}=1$ SUSY gauge theories and model building tools, Phys. Rev. D55 (1997), 7840-7858, hep-th/9612207.

[17] C. Csáki, M. Schmaltz, W. Skiba and J. Terning, Selfdual $\mathcal{N}=1$ SUSY gauge theories, Phys. Rev. D56 (1997), 1228-1238, hep-th/9701191.

[18] C. Csáki, W. Skiba and M. Schmaltz, Exact results and duality for $S P(2 N)$ SUSY gauge theories with an antisymmetric tensor, Nucl. Phys. B487 (1997), 128-140, hep-th/9607210.

[19] J. F. van Diejen and V. P. Spiridonov, An elliptic Macdonald-Morris conjecture and multiple modular hypergeometric sums, Math. Res. Lett 7 (2000), 729-746.

[20] J. F. van Diejen and V. P. Spiridonov, Elliptic Selberg integrals, Internat. Math. Res. Notices, no. 20 (2001), 1083-1110.

[21] J. F. van Diejen and V. P. Spiridonov, Unit circle elliptic beta integrals, Ramanujan J. 10 (2005), 187-204, math.CA/0309279.

[22] J. Distler and $\mathrm{A}$. Karch, $\mathcal{N}=1$ dualities for exceptional gauge groups and quantum global symmetries, Fortsch. Phys. 45 (1997), 517-533, hep-th/9611088.

[23] A. L. Dixon, On a generalisation of Legendre's formula $K E^{\prime}-(K-E) K^{\prime}=\frac{1}{2} \pi$, Proc. London Math. Soc. 2 (1) (1905), 206-224.

[24] F. A. Dolan, Counting BPS operators in $\mathcal{N}=4$ SYM, Nucl. Phys. B790 (2008), 432-464, arXiv:0704.1038 [hep-th].

[25] F. A. Dolan and H. Osborn, On short and semi-short representations for four dimensional superconformal symmetry, Ann. Phys. (NY) 307 (2003), 41-89, hep-th/0209056.

[26] F. A. Dolan and H. Osborn, Applications of the superconformal index for protected operators and $q$ hypergeometric identities to $\mathcal{N}=1$ dual theories, Nucl. Phys. B818 (2009), 137-178, arXiv:0801.4947 [hep-th].

[27] M. Eichler and D. Zagier, The Theory of Jacobi Forms, Progress in Math. 55, Birkhäuser, Boston, 1985.

[28] G. Felder and A. Varchenko, The elliptic gamma function and $S L(3, \mathbb{Z}) \ltimes \mathbb{Z}^{3}$, Adv. Math. 156 (2000), 44-76.

[29] B. Feng, A. Hanany and Y. H. He, Counting gauge invariants: the plethystic program, JHEP 0703 (2007) 090, hep-th/0701063.

[30] P. J. Forrester and S. O. Warnaar, The importance of the Selberg integral, Bull. Amer. Math. Soc. (N.S.) 45 (2008), 489-534, arXiv:0710.3981 [math.CA].

[31] E. Friedman and S. Ruijsenaars, Shintani-Barnes zeta and gamma functions, Adv. Math. 187 (2004), $362-395$.

[32] A. Gadde, E. Pomoni, L. Rastelli and S. S. Razamat, S-duality and 2d Topological QFT, JHEP 03 (2010) 032, arXiv:0910.2225 [hep-th].

[33] S. B. Giddings and J. M. Pierre, Some exact results in supersymmetric theories based on exceptional groups, Phys. Rev. D52 (1995), 6065-6073, hep-th/9506196.

[34] J. Gray, A. Hanany, Y. H. He, V. Jejjala and N. Mekareeya, SQCD: A geometric apercu, JHEP 0805 (2008) 099, arXiv:0803.4257 [hep-th].

[35] R. A. Gustafson, Some q-beta and Mellin-Barnes integrals with many parameters associated to the classical groups, SIAM J. Math. Anal. 23 (1992), 525-551.

[36] R. A. Gustafson, Some q-beta and Mellin-Barnes integrals on compact Lie groups and Lie algebras, Trans. AMS 341 (1) (1994), 69-119. 
[37] R. A. Gustafson, Some q-beta integrals on $S U(n)$ and $S p(n)$ that generalize the Askey-Wilson and Nassrallah-Rahman integrals, SIAM J. Math. Anal. 25 (1994), 441-449.

[38] R. A. Gustafson and M. A. Rakha, q-Beta integrals and multivariate basic hypergeometric series associated to root systems of type $A_{m}$, Ann. Comb. 4 (2000), 347-373.

[39] A. Hanany and N. Mekareeya, Counting gauge invariant operators in SQCD with classical gauge groups, JHEP 0810 (2008) 012, arXiv:0805.3728 [hep-th].

[40] G. 't Hooft, Naturalness, chiral symmetry, and spontaneous chiral symmetry breaking, Recent Developments in Gauge Theories (Eds. G. 't Hooft et. al.), Plenum Press, New York, 1980, pp. 135-157.

[41] K. Intriligator, New $R G$ fixed points and duality in supersymmetric $S P\left(N_{c}\right)$ and $S O\left(N_{c}\right)$ gauge theories, Nucl. Phys. B448 (1995), 187-198, hep-th/9505051.

[42] K. A. Intriligator, R. G. Leigh and M. J. Strassler, New examples of duality in chiral and nonchiral supersymmetric gauge theories, Nucl. Phys. B456 (1995), 567-621, hep-th/9506148.

[43] K. A. Intriligator and P. Pouliot, Exact superpotentials, quantum vacua and duality in supersymmetric SP $\left(N_{c}\right)$ gauge theories, Phys. Lett. B353 (1995), 471-476, hep-th/9505006.

[44] K. A. Intriligator and N. Seiberg, Phases of $\mathcal{N}=1$ supersymmetric gauge theories in four-dimensions, Nucl. Phys. B431 (1994), 551-568, hep-th/9408155.

[45] K. A. Intriligator and N. Seiberg, Duality, monopoles, dyons, confinement and oblique confinement in supersymmetric $S O\left(N_{c}\right)$ gauge theories, Nucl. Phys. B444 (1995), 125-160, hep-th/9503179.

[46] K. A. Intriligator and N. Seiberg, Phases of $\mathcal{N}=1$ supersymmetric gauge theories and electric - magnetic triality, Future perspectives in string theory, Los Angeles 1995, pp. 270-282, hep-th/9506084.

[47] K. A. Intriligator and N. Seiberg, Lectures on supersymmetric gauge theories and electric - magnetic duality, Nucl. Phys. Proc. Suppl. 45BC (1996), 1-28, hep-th/9509066.

[48] A. Karch, More on $\mathcal{N}=1$ selfdualities and exceptional gauge groups, Phys. Lett. B405 (1997), 280-286, hep-th/9702179.

[49] J. Kinney, J. M. Maldacena, S. Minwalla and S. Raju, An index for 4 dimensional super conformal theories, Commun. Math. Phys. 275 (2007), 209-254, hep-th/0510251.

[50] M. Klein, More confining $\mathcal{N}=1$ SUSY gauge theories from nonAbelian duality, Nucl. Phys. B553 (1999), 155-204, hep-th/9812155.

[51] D. Kutasov, A comment on duality in $\mathcal{N}=1$ supersymmetric non-Abelian gauge theories, Phys. Lett. B351 (1995), 230-234, hep-th/9503086.

[52] D. Kutasov and A. Schwimmer, On duality in supersymmetric Yang-Mills theory, Phys. Lett. B354 (1995), $315-321$, hep-th/9505004.

[53] D. Kutasov, A. Schwimmer and N. Seiberg, Chiral rings, singularity theory and electric-magnetic duality, Nucl. Phys. B459 (1996), 455-496, hep-th/9510222.

[54] R. G. Leigh and M. J. Strassler, Duality of $S p\left(2 N_{c}\right)$ and $S O\left(N_{c}\right)$ supersymmetric gauge theories with adjoint matter, Phys. Lett. B356 (1995), 492-499, hep-th/9505088.

[55] R. G. Leigh and M. J. Strassler, Accidental symmetries and $N=1$ duality in supersymmetric gauge theory, Nucl. Phys. B496 (1997), 132-148, hep-th/9611020.

[56] M. E. Machacek and M. T. Vaughn, Two loop renormalization group equations in a general quantum field theory. 1. Wave function renormalization, Nucl. Phys. B222 (1983), 83-103; 2. Yukawa couplings, ibid. B236 (1984), 221-232; 3. Scalar quartic couplings, ibid. B249 (1985), 70-92.

[57] Y. Nakayama, Index for orbifold quiver gauge theories, Phys. Lett. B636 (2006), 132-136, hep-th/0512280.

[58] N. A. Nekrasov, Seiberg-Witten prepotential from instanton counting, Adv. Theor. Math. Phys. 7 (2003), 831-864, hep-th/0206161.

[59] N. Nekrasov and A. Okounkov, Seiberg-Witten theory and random partitions, The Unity of Mathematics (Eds. P. Etingof, V. Retakh and I. M. Singer), Progr. Math., 244, Birkhauser, Boston, MA, 2006, pp. $525-596$, hep-th/0306238.

[60] I. Pesando, Exact results for the supersymmetric $G_{2}$ gauge theories, Mod. Phys. Lett. A10 (1995), 18711886, hep-th/9506139.

[61] P. Pouliot, Chiral duals of nonchiral SUSY gauge theories, Phys. Lett. B359 (1995), 108-113, hepth/9507018.

[62] P. Pouliot, Duality in SUSY SU(N) with an antisymmetric tensor, Phys. Lett. B367 (1996), 151-156, hep-th/9510148.

[63] P. Pouliot, Spectroscopy of gauge theories based on exceptional Lie groups, J. Phys. A34 (2001), 8631-8658, hep-th/0107151. 
[64] E. Poppitz and S. P. Trivedi, Some examples of chiral moduli spaces and dynamical supersymmetry breaking, Phys. Lett. B365 (1996), 125-131, hep-th/9507169.

[65] E. M. Rains, Transformations of elliptic hypergeometric integrals, Ann. of Math. 171 (2010), 169-243, math.QA/0309252v4.

[66] E. M. Rains, $B C_{n}$-symmetric abelian functions, Duke Math. J. 135 (1) (2006), 99-180, math.CO/0402113v2.

[67] E. M. Rains, Limits of elliptic hypergeometric integrals, Ramanujan J. 18 (3) (2009), 257-306, math.CA/0607093.

[68] E. M. Rains, Elliptic Littlewood identities, arXiv:0806.0871 [math.CO].

[69] E. M. Rains and V. P. Spiridonov, Determinants of elliptic hypergeometric integrals, Funkt. Analiz i ego Pril. 43 (4) (2009), 67-86 (Funct. Analysis and its Appl. 43 (4) (2009), 297-311), arXiv:0712.4253 [math.CA].

[70] P. Ramond, Superalgebras in $\mathcal{N}=1$ gauge theories, Phys. Lett. B390 (1997), 179-184, arXiv:hepth/9608077.

[71] A. G. Reiman, M. A. Semenov-Tian-Shansky and L. D. Faddeev, Quantum anomalies and cocycles on gauge groups, Funkt. Analiz i ego Pril. 18 (4) (1984), 64-72 (Funct. Analysis and its Appl. 18 (4) (1984), 319-326).

[72] C. Römelsberger, Counting chiral primaries in $\mathcal{N}=1, d=4$ superconformal field theories, Nucl. Phys. B747 (2006), 329-353, hep-th/0510060.

[73] C. Römelsberger, Calculating the superconformal index and Seiberg duality, arXiv:0707.3702 [hep-th].

[74] S. N. M. Ruijsenaars, First order analytic difference equations and integrable quantum systems, J. Math. Phys. 38 (1997), 1069-1146.

[75] N. Seiberg, Exact results on the space of vacua of four-dimensional SUSY gauge theories, Phys. Rev. D49 (1994), 6857-6863, hep-th/9402044.

[76] N. Seiberg, Electric-magnetic duality in supersymmetric non-Abelian gauge theories, Nucl. Phys. B435 (1995), 129-146, hep-th/9411149.

[77] N. Seiberg and E. Witten, Electric - magnetic duality, monopole condensation, and confinement in $\mathcal{N}=2$ supersymmetric Yang-Mills theory, Nucl. Phys. B426 (1994), 19-52, Erratum-ibid. B430 (1994), 485-486, hep-th/9407087.

[78] N. Seiberg and E. Witten, Monopoles, duality and chiral symmetry breaking in $\mathcal{N}=2$ supersymmetric $Q C D$, Nucl. Phys. B431 (1994), 484-550, hep-th/9408099.

[79] M. A. Shifman, Nonperturbative dynamics in supersymmetric gauge theories, Prog. Part. Nucl. Phys. 39 (1997), 1-116, hep-th/9704114.

[80] B. S. Skagerstam, On the large $N_{c}$ limit of the $S U\left(N_{c}\right)$ colour quark-gluon partition function, Z. Phys. C24 (1984), 97-101.

[81] V. P. Spiridonov, On the elliptic beta function, Uspekhi Mat. Nauk 56 (1) (2001), 181-182 (Russian Math. Surveys 56 (1) (2001), 185-186).

[82] V. P. Spiridonov, Theta hypergeometric series, Proceedings of the NATO ASI Asymptotic Combinatorics with Applications to Mathematical Physics (St. Petersburg, Russia, July 2001), Eds. V. A. Malyshev and A. M. Vershik, Kluwer, 2002, pp. 307-327, math.CA/0303204.

[83] V. P. Spiridonov, Theta hypergeometric integrals, Algebra i Analiz 15 (6) (2003), 161-215 (St. Petersburg Math. J. 15 (6) (2003), 929-967), math.CA/0303205.

[84] V. P. Spiridonov, A Bailey tree for integrals, Teor. Mat. Fiz. 139 (2004), 104-111 (Theor. Math. Phys. 139 (2004), 536-541), math.CA/0312502.

[85] V. P. Spiridonov, Elliptic hypergeometric functions, Habilitation thesis, Bogoliubov Laboratory of Theoretical Physics, JINR, September 2004, 218 pp. (Russian).

[86] V. P. Spiridonov, Short proofs of the elliptic beta integrals, Ramanujan J. 13 (2007), 265-283, math.CA/0408369.

[87] V. P. Spiridonov, Elliptic hypergeometric functions and Calogero-Sutherland type models, Teor. Mat. Fiz, 150 (2) (2007), 311-324 (Theor. Math. Phys. 150 (2) (2007), 266-277).

[88] V. P. Spiridonov, Continuous biorthogonality of the elliptic hypergeometric function, Algebra i Analiz (St. Petersburg Math. J.) 20 (5) (2008), 155-185, arXiv:0801.4137 [math.CA].

[89] V. P. Spiridonov, Essays on the theory of elliptic hypergeometric functions, Uspekhi Mat. Nauk 63 (3) (2008), 3-72 (Russian Math. Surveys 63 (3) (2008), 405-472), arXiv:0805.3135 [math.CA].

[90] V. P. Spiridonov, Elliptic hypergeometric terms, Proc. of the Workshop "Théories galoisiennes et arithmétiques des équations différentielles" (September 2009, CIRM, Luminy, France), to appear, arXiv:1003.4491 [math.CA]. 
[91] V. P. Spiridonov and G. S. Vartanov, Superconformal indices for $\mathcal{N}=1$ theories with multiple duals, Nucl. Phys. B824 (2010), 192-216, arXiv:0811.1909v4 [hep-th].

[92] V. P. Spiridonov and G. S. Vartanov, Supersymmetric dualities beyond the conformal window, Phys. Rev. Lett. 105 (2010) 061603, arXiv:1003.6109 [hep-th].

[93] V. P. Spiridonov and S. O. Warnaar, Inversions of integral operators and elliptic beta integrals on root systems, Adv. Math. 207 (2006), 91-132, math.CA/0411044.

[94] V. P. Spiridonov and S. O. Warnaar, New multiple ${ }_{6} \psi_{6}$ summation formulas and related conjectures, Ramanujan J., to appear.

[95] B. Sundborg, The Hagedorn transition, deconfinement and $\mathcal{N}=4$ SYM theory, Nucl. Phys. B573 (2000), 349-363, hep-th/9908001.

[96] L. A. Takhtadzhyan and L. D. Faddeev, The quantum method of the inverse problem and the Heisenberg XYZ model, Uspekhi Mat. Nauk 34 (5) (1979), 13-63 (Russian Math. Surveys 34 (5) (1979), 11-68).

[97] J. Terning, Modern Supersymmetry: Dynamics and Duality, Oxford, UK: Clarendon (2006), 324 pp.

[98] A. Yu. Volkov, Noncommutative hypergeometry, Commun. Math. Phys. 258 (2005), 257-273, math.CA/0312084.

[99] E. Witten, Constraints on supersymmetry breaking, Nucl. Phys. B202 (1982), 253-316.

Bogoliubov laboratory of Theoretical Physics, JinR, Dubna, Moscow Region 141980, RusSia And Theory Division, INR RAS, Moscow, Russia; E-MaIl ADdREss: SPIRIDOn@theor.JinR.RU

Max-Planck-Institut für Gravitationsphysik, Albert-Einstein-Institut 14476 Golm, GerMANY; E-MAIL ADDRESS: VARTANOV@AEI.MPG.DE 